\title{
Characterization of the Quasi-Stationary State of an Impurity Driven by Monochromatic Light II - Microscopic Foundations
}

\author{
J.-B. Bru and W. de Siqueira Pedra
}

August 9, 2021

\begin{abstract}
From quantum mechanical first principles only, we rigorously study the time-evolution of a $N$-level atom (impurity) interacting with an external monochromatic light source within an infinite system of free electrons at thermal equilibrium (reservoir). In particular, we establish the relation between the full dynamics of the compound system and the effective dynamics for the $N$-level atom, which is studied in detail in [1]. Together with [1] the present paper yields a purely microscopic theory of optical pumping in laser physics. The model we consider is general enough to describe gauge invariant atom-reservoir interactions.
\end{abstract}

\section{INTRODUCTION}

Optical pumping is an important method in laser technology to produce the so-called "inversion of population" of some optically active (quantum) system, as for instance impurities in crystals 2, 3. Such an inversion is then used to obtain optical amplification through stimulated emission of photons. We aim to derive the phenomenon of optical pumping by (i) being coherent with the phenomenological description of physics textbooks and experimental facts, (ii) starting from first principles of quantum mechanics only, and applying mathematically rigorous methods to study microscopic models.

We analyzed in [1] the effective time-evolution of a $N$-level atom (an impurity) interacting with an external monochromatic light source within a host environment (reservoir), which is represented by an infinite system of free fermions at thermal equilibrium. The external monochromatic light source is a time-periodic classical field stimulating transitions between two energy levels of the impurity. We showed [1 from this effective dynamics how an inversion of population of energy levels of the impurity can appear and derive a dynamical law for the evolution of populations under the influence of the external oscillating field (optical pumping). We proved [1] that a generalization of the celebrated Pauli master equation, used in all standard textbooks on laser physics, correctly describes the time-evolution of populations. In contrast to the usual Pauli equation, this generalization takes memory effects into account. This proof uses [1, Theorem 3.3], which states that the restriction of the full unitary dynamics (of the impurity-reservoir-pump system) to the $N$-level atom is properly described - up to small corrections for moderate pump strengths - by an effective non-autonomous time-evolution involving atomic degrees of freedom only. The detailed proof of this assertion, which is not performed in 1, is the main issue of the present paper.

Thus, together with [1, the results presented here give a complete microscopic derivation of optical pumping and the induced inversion of population from quantum mechanical first principles only. Indeed, to our knowledge there is only one framework in which aspects of laser phenomenology has been mathematically rigorously analyzed from first principles, namely for some versions of the Dicke model [4, see [5, 6, 7, 8]. Nevertheless, Dicke-type models are based on two-level atoms whereas the phenomenology of lasers as described in physics textbooks is based on three- or four-level atoms [9. Moreover, they cannot explain the inversion of population at finite number of impurities. For more details, see [1, Section 1] as well as [10, Chap. 11]. Note that so-called "one-atom lasers" are object of recent research, both experimentally and theoretically. See for instance [11. In a future work, we aim to couple the impurity-reservoir-pump system considered here to a cavity (few-mode bosonic field) in order to study light amplification in such devices, directly from the microscopic quantum dynamics. 
The derivation of the effective atomic dynamics is conceptually similar to what is done in [1, Section 4], but technically more involved: We represent the non-autonomous evolution as an autonomous dynamics on some enlarged Hilbert space of periodic functions (Floquet-Howland method). Next, we perform an analytic translation $\mathcal{G}(\theta)$ of the generator $\mathcal{G}$ of the autonomous dynamics and prove that the dynamics driven by both operators coincide with each other when restricted to the atomic subspace. We then study the discrete spectrum and eigenspaces of $\mathcal{G}(\theta)$ through Kato's perturbation theory [12. Finally, by using the inverse Laplace transform for strongly continuous semigroups together with Riesz projections, we analyze the action of the semigroup $\left\{\mathrm{e}^{\alpha \mathcal{G}(\theta)}\right\}_{\alpha \geq 0}$ on vectors of the atomic subspace. This analysis leads to the main result of the paper, that is, Theorem [3.1. Notice that, as compared to the model used in [1 to illustrate the microscopic origin of the effective dissipative dynamics of the impurity, we consider here a more general atom-reservoir coupling in order to include gauge invariant interactions. The results of [1] only concern the effective dynamics of the impurity and are very general. They also hold for such more physical microscopic interactions.

To finish, we would like to drive the attention of the reader to [13 which complements the present work in the following sense: The model considered in [13] is exactly the same one treated here, up to the fact that the time-dependent perturbation $H_{c}(t)$ of the atomic part commutes with the unperturbed atomic Hamiltonian $H_{c}$ and is very strong. See [13, Section "model and main results"]. By contrast, in the situation considered here the time-dependent perturbation $\eta H_{\mathrm{p}}(t)$ does not commute with the atomic Hamiltonian $H_{\text {at }}$ and is very weak. See Section 2 below. Moreover, here we obtain an effective purely atomic dynamics which well approximates the full evolution of populations uniformly for all times, whereas in [13] only the time scale $t \sim(\lambda T)^{-1}$ is considered. In [13, as well as in the present work, $\lambda$ denotes the reservoir-atom coupling and is small, but not vanishing. $T$ stands in 13 for the time-period of the "control term" $H_{c}(t)$ and the regime of interest is the limit $T \downarrow 0$ with $H_{c}(t)=\mathcal{O}\left(T^{-1}\right)$. By contrast, here the time-period of $H_{\mathrm{p}}(t)$ is fixed once for all (the pump frequency is set to be equal to the largest Bohr frequency of the atom) and the pump-atom coupling $\eta$ is of order $\mathcal{O}\left(\lambda^{2}\right)$. Mathematical methods like time-dependent $C$-Liouvilleans, evolution groups and Howland operators, complex spectral deformation, Riesz projections, and others used in [13] will be again employed here. Because of the big difference between the analyzed regimes explained above, note however that both studies differ from each other in which concerns technical aspects.

The paper is organized as follows. In Section 2 we introduce the microscopic model. Then, in Section 3 we define the dynamics of the impurity-reservoir-pump system and state our main result (Theorem 3.1), which is proven in Section 4. Finally, Section [5 is an appendix briefly reviewing, for the reader's convenience, some useful mathematical objects.

\section{Notation 1.1}

To simplify notation, we denote by $C, D \in \mathbb{R}^{+}$generic positive and finite constants. Note that these constants do not need to be the same from one statement to another. A norm on space $\mathcal{X}$ is denoted by $\|\cdot\| \mathcal{X}$. A similar notation is used for scalar products in Hilbert spaces. $\mathbf{1}_{\mathcal{X}}$ denotes either the identity of a $C^{*}$-algebra $\mathcal{X}$ or the identity operator acting on $\mathcal{X}$.

\section{ThE HOST ENVIRONMENT-IMPURITY-LIGHT SOURCE MICROSCOPIC MODEL}

For completeness and to fix notations, we recall below the setting of [1. We keep the discussions as short as possible and refer to [1, Sections 2-3] for details.

\subsection{The host environment as a thermal reservoir of free fermions}

Let $\mathfrak{h}_{1}:=L^{2}\left(\mathbb{R}^{3}\right)$ and $\mathbf{E}: \mathbb{R}^{3} \rightarrow \mathbb{R}$ be any measurable rotationally invariant function that, up to some diffeomorphism, behaves like $|p|$. In the sequel, to simplify discussions, we set $\mathbf{E}(p)=|p|$. Define the (multiplication) operator $h_{1}=h_{1}(\mathbf{E})$ on $\mathfrak{h}_{1}$ by $f(p) \mapsto \mathbf{E}(p) f(p)$. Let $\mathcal{V}_{\mathcal{R}}$ be the CAR $C^{*}$-algebra generated by the annihilation and creation operators $a(f), a^{+}(f):=a(f)^{*}, f \in \mathfrak{h}_{1}$, acting on the antisymmetric Fock space $\mathcal{F}_{-}\left(\mathfrak{h}_{1}\right)$ (with one-particle Hilbert space $\left.\mathfrak{h}_{1}\right)$ and fulfilling canonical anti-commutation relations. The dynamics of the reservoir is given by the strongly continuous group $\left\{\tau_{t}^{\mathcal{R}}\right\}_{t \in \mathbb{R}}$ of Bogoliubov automorphisms on $\mathcal{V}_{\mathcal{R}}$ uniquely defined by the condition:

$$
\tau_{t}^{\mathcal{R}}(a(f))=a\left(\mathrm{e}^{i t h_{1}} f\right), \quad f \in \mathfrak{h}_{1}, \quad t \in \mathbb{R} .
$$


$\delta_{\mathcal{R}}$ denotes the symmetric derivation generating $\tau_{t}^{\mathcal{R}}$. The initial state of the reservoir $\omega_{\mathcal{R}}$ at inverse temperature $\beta \in \mathbb{R}^{+}$is the unique $\left(\tau^{\mathcal{R}}, \beta\right)-\mathrm{KMS}$ state (thermal equilibrium state).

\subsection{The impurity as a $N$-level atom}

Let $d \in \mathbb{N}$ and $\mathcal{B}\left(\mathbb{C}^{d}\right)$ be the finite dimensional $C^{*}$-algebra of all linear operators on $\mathbb{C}^{d}$ and take any selfadjoint element $H_{\text {at }}=H_{\text {at }}^{*} \in \mathcal{B}\left(\mathbb{C}^{d}\right)$ with $H_{\text {at }} \notin \mathbb{R} \mathbf{1}_{\mathcal{B}\left(\mathbb{C}^{d}\right)} \subset \mathcal{B}\left(\mathbb{C}^{d}\right)$. Eigenvalues and eigenspaces of $H_{\text {at }}$ are denoted by $E_{k} \in \mathbb{R}$ and $\mathcal{H}_{k} \subset \mathbb{C}^{d}, k \in\{1, \ldots, N\}(N \geq 2)$, respectively. $E_{k}$ is chosen such that $E_{j}<E_{k}$ whenever $j<k$. The dimension $n_{k}$ of the eigenspace $\mathcal{H}_{k}$ is the degeneracy of the $k$ th atomic level. Then, the free atomic dynamics is given by the group $\tau^{\text {at }}:=\left\{\tau_{t}^{\text {at }}\right\}_{t \in \mathbb{R}}$ of automorphisms of the $C^{*}$-algebra $\mathcal{B}\left(\mathbb{C}^{d}\right)$ defined by

$$
\tau_{t}^{\text {at }}(A):=\mathrm{e}^{i t H_{\text {at }}} A \mathrm{e}^{-i t H_{\text {at }}}, \quad A \in \mathcal{B}\left(\mathbb{C}^{d}\right),
$$

for all $t \in \mathbb{R}$.

Let $\omega_{\text {at }}$ be any faithful state on $\mathcal{B}\left(\mathbb{C}^{d}\right)$ and denote by $\rho_{\text {at }} \in \mathcal{B}\left(\mathbb{C}^{d}\right)$ its unique density matrix, i.e.,

$$
\omega_{\mathrm{at}}(A)=\operatorname{Tr}_{\mathbb{C}^{d}}\left(\rho_{\mathrm{at}} A\right), \quad A \in \mathcal{B}\left(\mathbb{C}^{d}\right)
$$

For any inverse temperature $\beta \in \mathbb{R}^{+}$, the thermal equilibrium state of the (free) atom is the (Gibbs) state $\mathfrak{g}_{\mathrm{at}}^{(\beta)} \equiv \mathfrak{g}_{\mathrm{at}}$ associated to the density matrix

$$
\rho_{\mathfrak{g}}:=\frac{\mathrm{e}^{-\beta H_{\mathrm{at}}}}{\operatorname{Tr}_{\mathbb{C}^{d}}\left(\mathrm{e}^{-\beta H_{\mathrm{at}}}\right)} .
$$

The triplet $\left(\mathfrak{H}_{\mathrm{at}}, \pi_{\mathrm{at}}, \Omega_{\mathrm{at}}\right)$ stands for the standard GNS representation of $\omega_{\mathrm{at}}$ :

$$
\mathfrak{H}_{\mathrm{at}}:=\left(\mathcal{B}\left(\mathbb{C}^{d}\right),\langle\cdot, \cdot\rangle_{\mathfrak{H}_{\mathrm{at}}}\right)
$$

with

$$
\langle A, B\rangle_{\mathfrak{H}_{\mathrm{at}}}:=\operatorname{Tr}_{\mathbb{C}^{d}}\left(A^{*} B\right), \quad A, B \in \mathcal{B}\left(\mathbb{C}^{d}\right)
$$

while

$$
\Omega_{\mathrm{at}}:=\rho_{\mathrm{at}}^{1 / 2} \in \mathfrak{H}_{\mathrm{at}} \quad \text { and } \quad \pi_{\mathrm{at}}(A)=\stackrel{A}{\rightarrow}, \quad A \in \mathcal{B}\left(\mathbb{C}^{d}\right) .
$$

Here, for any $A \in \mathcal{B}\left(\mathbb{C}^{d}\right)$, the left and right multiplication operators $\stackrel{A}{\rightarrow}$ and $\_$are respectively defined on $\mathcal{B}\left(\mathbb{C}^{d}\right)$ by

$$
B \mapsto \underset{A}{\rightarrow} B:=A B \quad \text { and } \quad B \mapsto \not \subset B:=B A .
$$

Note that the dynamics of the atom defined by (2.2) can be represented in the Schrödinger picture of Quantum Mechanics through the Lindbladian

$$
\mathfrak{L}_{\mathrm{at}}:=-i\left(\stackrel{H_{\mathrm{at}}}{\longrightarrow}-\underset{H_{\mathrm{at}}}{\longleftarrow}\right)=-i\left[H_{\mathrm{at}}, \cdot\right]=-\mathfrak{L}_{\mathrm{at}}^{*}
$$

acting on the Hilbert space $\mathfrak{H}_{\text {at }}$ since, for all $t \in \mathbb{R}$,

$$
\omega_{\text {at }}\left(\tau_{t}^{\text {at }}(A)\right)=\left\langle\mathrm{e}^{t \mathfrak{L}_{\mathrm{at}}} \Omega_{\mathrm{at}}, \pi_{\mathrm{at}}(A) \mathrm{e}^{t \mathcal{L}_{\mathrm{at}}} \Omega_{\mathrm{at}}\right\rangle_{\mathfrak{H}_{\mathrm{at}}}, \quad A \in \mathcal{B}\left(\mathbb{C}^{d}\right)
$$

Define the sets

$$
\mathfrak{t}_{\epsilon}:=\left\{(j, k): E_{j}-E_{k}=\epsilon\right\} \subset\{1,2, \ldots, N\} \times\{1,2, \ldots, N\}
$$

for each eigenvalue

$$
\epsilon \in \sigma\left(i \mathfrak{L}_{\mathrm{at}}\right)=\left\{E_{j}-E_{k}: j, k \in\{1,2, \ldots, N\}\right\} .
$$

Here, $\sigma(A)$ denotes the spectrum of the operator $A$. Furthermore, for all $\epsilon \in \sigma\left(i \mathfrak{L}_{\text {at }}\right)$, denote by $P_{\text {at }}^{(\epsilon)} \in \mathcal{B}\left(\mathfrak{H}_{\text {at }}\right)$ the orthogonal projection on $\mathfrak{H}_{\text {at }}$ associated to the eigenspace of $i \mathfrak{L}_{\text {at }}$ with eigenvalue $\epsilon$. $\mathcal{B}\left(\mathfrak{H}_{\text {at }}\right)$ stands for the set of all linear operators on $\mathfrak{H}_{\text {at }}$. 


\subsection{The uncoupled reservoir-atom system}

Define the $C^{*}$-algebra $\mathcal{V}:=\mathcal{B}\left(\mathbb{C}^{d}\right) \otimes \mathcal{V}_{\mathcal{R}}$. Then the free dynamics of the atom-reservoir compound system is given by the strongly continuous group $\tau:=\left\{\tau_{t}\right\}_{t \in \mathbb{R}}$ of automorphisms of $\mathcal{V}$ defined by

$$
\tau_{t}:=\tau_{t}^{\text {at }} \otimes \tau_{t}^{\mathcal{R}}, \quad t \in \mathbb{R} .
$$

The generator of this dynamics is the symmetric derivation denoted by $\delta$ and acts on a dense sub-*-algebra $\operatorname{Dom}(\delta)$ of $\mathcal{V}$. The initial state of the atom-reservoir system is

$$
\omega_{0}:=\omega_{\text {at }} \otimes \omega_{\mathcal{R}} .
$$

As the state $\omega_{0}$ is faithful, the map $\mathcal{V} \times \mathcal{V} \rightarrow \mathbb{C}$,

$$
(A, B) \mapsto \omega_{0}\left(A^{*} B\right),
$$

defines a scalar product on $\mathcal{V}$. For any fixed inverse temperature $\beta \in \mathbb{R}^{+}$, set $\omega_{\mathrm{at}}:=\mathfrak{g}_{\mathrm{at}}^{(\beta)}$ and let the Hilbert space $\mathfrak{H}^{(\beta)}$ be the completion of $\mathcal{V}$ with respect to (w.r.t.) the above scalar product. $\tilde{\mathfrak{P}}_{\text {at }}^{(\beta)}$ stands for the orthogonal projection on $\mathfrak{H}^{(\beta)}$ with (finite dimensional) range

$$
\operatorname{ran}\left(\tilde{\mathfrak{P}}_{\mathrm{at}}^{(\beta)}\right)=\mathcal{B}\left(\mathbb{C}^{d}\right) \otimes \mathbf{1}_{\mathcal{V}_{\mathcal{R}}} \subset \mathcal{V} \subset \mathfrak{H}^{(\beta)} .
$$

As $\operatorname{ran}\left(\tilde{\mathfrak{P}}_{\text {at }}^{(\beta)}\right) \subset \mathcal{V} \subset \mathfrak{H}^{(\beta)}$, the restriction $\tilde{\mathfrak{P}}_{\text {at }}^{(\beta)}$ to $\mathcal{V}$ defines a projection $\mathfrak{P}_{\text {at }}^{(\beta)} \equiv \mathfrak{P}_{\text {at }}$ on $\mathcal{V}$. Notice that in the sequel we identify $\operatorname{ran}\left(\mathfrak{P}_{\text {at }}^{(\beta)}\right)=\mathcal{B}\left(\mathbb{C}^{d}\right) \otimes \mathbf{1}_{\mathcal{V}_{\mathcal{R}}} \subset \mathcal{V}$ and $\mathcal{B}\left(\mathbb{C}^{d}\right)$.

\subsection{Classical optical pump}

Define

$$
H_{\mathrm{p}}:=h_{\mathrm{p}}+h_{\mathrm{p}}^{*} \in \mathcal{B}\left(\mathbb{C}^{d}\right)
$$

for some $h_{\mathrm{p}} \in \mathcal{B}\left(\mathbb{C}^{d}\right)$ satisfying

$$
\begin{aligned}
\operatorname{ker}\left(h_{\mathrm{p}}\right)^{\perp} & \subseteq \mathcal{H}_{1}:=\operatorname{ran}\left(\mathbf{1}\left[H_{\mathrm{at}}=E_{1}\right]\right) \\
\operatorname{ran}\left(h_{\mathrm{p}}\right) & \subseteq \mathcal{H}_{N}:=\operatorname{ran}\left(\mathbf{1}\left[H_{\mathrm{at}}=E_{N}\right]\right) .
\end{aligned}
$$

Here, $\mathbf{1}\left[H_{\mathrm{at}}=E\right]$ stands for the orthogonal projection on the eigenspace of $H_{\mathrm{at}}$ with corresponding eigenvalue $E$. The coupling of the optical pump to the atom is represented by a time-dependent perturbation of the form

$$
\eta \cos (\varpi t) \mathfrak{L}_{\mathrm{p}}, \quad \varpi:=E_{N}-E_{1}>0, \quad t \in \mathbb{R},
$$

to the atomic Lindbladian $\mathfrak{L}_{\text {at }}$. Here,

$$
\mathfrak{L}_{\mathrm{p}}:=-i\left(\stackrel{H_{\mathrm{p}}}{\longrightarrow}-\underset{H_{\mathrm{p}}}{\longleftarrow}\right)=-i\left[H_{\mathrm{p}}, \cdot\right]=-\mathfrak{L}_{\mathrm{p}}^{*}
$$

and $\eta \in \mathbb{R}$ is a coupling constant.

\subsection{Field form factors of the atom-reservoir interaction}

Let $\mathrm{K}, m \in \mathbb{N}$ and, for any $\kappa=\{1, \ldots, \mathrm{K}\}$, let $\left\{f_{\ell}^{(\kappa)}\right\}_{\ell=1}^{m} \subset \mathfrak{h}_{1}$ be a family of rotationally invariant functions, i.e., $f_{\ell}^{(\kappa)}(p)=\mathrm{f}_{\ell}^{(\kappa)}(|p|)$ for all $p \in \mathbb{R}^{3}$ and $\ell \in\{1, \ldots, m\}$ with $\mathrm{f}_{\ell}^{(\kappa)}: \mathbb{R}_{0}^{+} \rightarrow \mathbb{C}$. For all $\kappa=\{1, \ldots, \mathrm{K}\}$ and $\ell \in\{1, \ldots, m\}$, the complex-valued functions $\mathrm{g}_{\ell}^{(\kappa)}$ and $\mathrm{g}_{\ell}^{(\kappa) \#}$ on $\mathbb{R}$ are respectively defined by

$$
\forall x \in \mathbb{R}: \quad \mathrm{g}_{\ell}^{(\kappa)}(x):=|x|\left(1+\mathrm{e}^{-\beta x}\right)^{-1 / 2} \begin{cases}\mathrm{f}_{\ell}^{(\kappa)}(x) & , x \geq 0, \\ \frac{\mathrm{f}_{\ell}^{(\kappa)}(-x)}{,} & x<0,\end{cases}
$$

and

We assume the following:

$$
\mathrm{g}_{\ell}^{(\kappa) \#}(x):=\overline{i \mathrm{~g}_{\ell}^{(\kappa)}(-x)}, \quad x \in \mathbb{R} .
$$




\section{Assumption 1}

There is $\mathrm{r}_{\max }>0$ such that $\mathrm{g}_{\ell}^{(\kappa)}$ and $\mathrm{g}_{\ell}^{(\kappa) \#}$ have an analytic continuation to the strip $\mathbb{R}+i\left(-\mathrm{r}_{\max }, \mathrm{r}_{\max }\right)$ and satisfy

$$
\sup _{y \in\left(-\mathrm{r}_{\max }, \mathrm{r}_{\max }\right)}\left\{\int_{\mathbb{R}}\left(\left|\mathrm{g}_{\ell}^{(\kappa)}(x+i y)\right|+\left|\mathrm{e}^{-\frac{\beta}{2}(x+i y)} \mathrm{g}_{\ell}^{(\kappa) \#}(x+i y)\right|\right)^{2} \mathrm{~d} x\right\}<\infty .
$$

[The factor $\mathrm{e}^{-\frac{\beta}{2}(x+i y)}$ in this assumption is, by mistake, missing in the condition below [1, Eq. (2.16)].]

To satisfy this condition one may, for instance, choose the functions $\mathrm{f}_{\ell}^{(\kappa)}(x)$ as linear combinations of terms of the form $|x|^{2 p-1} \exp \left(-C x^{2}\right)$ with $p \in \mathbb{N}_{0}$. Finally, at any fixed inverse temperature $\beta \in \mathbb{R}^{+}$of the fermionic reservoir and for any $\kappa=\{1, \ldots, \mathrm{K}\}$, let $\left\{f_{\ell}^{(\kappa, \beta)}\right\}_{\ell=1}^{m}$ be the family of functions $\mathbb{R} \rightarrow \mathbb{R}_{0}^{+}$defined by

$$
f_{\ell}^{(\kappa, \beta)}(x):=4 \pi \frac{\left|x \mathrm{f}_{\ell}^{(\kappa)}(|x|)\right|^{2}}{1+\mathrm{e}^{-\beta x}}=4 \pi\left|\mathrm{g}_{\ell}^{(\kappa)}(x)\right|^{2} .
$$

\subsection{Atom-reservoir interaction}

For all $\kappa \in\{1, \ldots, \mathrm{K}\}$ choose a finite collection $\left\{Q_{\ell_{1}, \ldots, \ell_{k}}^{(\kappa)}\right\}_{\ell_{1}, \ldots, \ell_{k}=1}^{m} \subset \mathcal{B}\left(\mathbb{C}^{d}\right)$ satisfying

$$
\left(Q_{\ell_{1}, \ldots, \ell_{\kappa}}^{(\kappa)}\right)^{*}=Q_{\ell_{\kappa}, \ldots, \ell_{1}}^{(\kappa)}
$$

Then, the atom-reservoir interaction is implemented by the bounded symmetric derivation $\lambda \delta_{\text {at }, \mathcal{R}}$ with coupling constant $\lambda \in \mathbb{R}$ and

$$
\delta_{\mathrm{at}, \mathcal{R}}:=i \sum_{\kappa=1}^{\mathrm{K}} \sum_{\ell_{1}, \ldots, \ell_{\kappa}=1}^{m}\left[Q_{\ell_{1}, \ldots, \ell_{\kappa}}^{(\kappa)} \otimes \Phi\left(f_{\ell_{1}}^{(\kappa)}\right) \cdots \Phi\left(f_{\ell_{\kappa}}^{(\kappa)}\right), \cdot\right]
$$

Here, for all $f \in \mathfrak{h}_{1}$,

$$
\Phi(f):=\frac{1}{\sqrt{2}}\left(a^{+}(f)+a(f)\right)=\Phi(f)^{*} \in \mathcal{B}\left(\mathcal{F}_{-}\left(\mathfrak{h}_{1}\right)\right) .
$$

For any normed space $\mathcal{X}, \mathcal{B}(\mathcal{X})$ stands for the set of all linear bounded operators on $\mathcal{X}$.

To simplify discussions and proofs, as also done by other authors in similar situations (see, for instance, 14 , Section 2.2, Assumption 2.4]), without loss of generality (w.l.o.g.), we assume:

\section{Assumption $2 \quad \mathfrak{P}_{\mathrm{at}}^{(\beta)} \delta_{\mathrm{at}, \mathcal{R} \mathfrak{P}_{\mathrm{at}}^{(\beta)}=0 .}$}

This technical condition is not essential for the results below and does not exclude most physically relevant atom-reservoir couplings. Note that this condition is automatically fulfilled if $\delta_{\mathrm{at}, \mathcal{R}}$ is a linear combination of odd monomials in the fermionic fields $\Phi(f), f \in \mathfrak{h}_{1}$. This is the case for the model considered in [1] and hence, this technical condition is not explicitly imposed there. Moreover, Assumption 2 does not force the component of $\delta_{\mathrm{at}, \mathcal{R}}$ which is even w.r.t. to the fermionic fields to vanish.

For all $\varepsilon \in \mathbb{R}^{+}$, define the linear operator $\mathcal{L}_{\mathcal{R}}^{(\varepsilon)} \in \mathcal{B}\left(\mathfrak{H}_{\mathrm{Hat}}\right)$ by

$$
\mathfrak{L}_{\mathcal{R}}^{(\varepsilon)}:=\sum_{\epsilon \in \sigma\left(i \mathfrak{L}_{\mathrm{at}}\right)}\left(P_{\mathrm{at}}^{(\epsilon)} \mathfrak{P}_{\mathrm{at}}^{(\beta)} \delta_{\mathrm{at}, \mathcal{R}}\left(1-\mathfrak{P}_{\mathrm{at}}^{(\beta)}\right)(\varepsilon+i \epsilon-\delta)^{-1}\left(1-\mathfrak{P}_{\mathrm{at}}^{(\beta)}\right) \delta_{\mathrm{at}, \mathcal{R} \mathfrak{P}_{\mathrm{at}}^{(\beta)}} P_{\mathrm{at}}^{(\epsilon)}\right)^{*}
$$

Recall that we identify the spaces $\operatorname{ran}\left(\mathfrak{P}_{\mathrm{at}}^{(\beta)}\right)=\mathcal{B}\left(\mathbb{C}^{d}\right) \otimes \mathbf{1}_{\mathcal{V}_{\mathcal{R}}} \subset \mathcal{V}$ and $\mathcal{B}\left(\mathbb{C}^{d}\right)$. Note further that $\varepsilon, \epsilon \in \mathbb{R}$ and $\sigma(\delta) \subset i \mathbb{R}$. [ $\delta$ generates a group of contractions, i.e., $\pm \delta$ are generators of semigroups of contractions.] From the analyticity assumptions on the field form factors of the atom-reservoir interactions, one shows that the following limit exists:

$$
\mathfrak{L}_{\mathcal{R}}:=\lim _{\varepsilon \searrow 0} \mathfrak{L}_{\mathcal{R}}^{(\varepsilon)} \in \mathcal{B}\left(\mathfrak{H}_{\mathrm{at}}\right) \equiv \mathcal{B}\left(\mathcal{B}\left(\mathbb{C}^{d}\right)\right)
$$


See for instance Lemma 4.13. It is known that, in this case, the limit $\mathcal{L}_{\mathcal{R}}$ is the generator of a completely positive group, see for instance [14, Section 6.1]. By the Stinespring theorem,

$$
\mathfrak{L}_{\mathcal{R}}=-i\left(\stackrel{H_{\text {Lamb }}}{\stackrel{H_{\text {Lamb }}}{\longleftarrow}}\right)+\mathfrak{L}_{d}
$$

where $H_{\text {Lamb }}=H_{\text {Lamb }}^{*} \in \mathcal{B}\left(\mathbb{C}^{d}\right)$ and $\mathfrak{L}_{d}$ has the form

$$
\mathfrak{L}_{d}(\rho)=\sum_{\alpha \in \mathrm{A}}\left(2 V_{\alpha} \rho V_{\alpha}^{*}-V_{\alpha}^{*} V_{\alpha} \rho-\rho V_{\alpha}^{*} V_{\alpha}\right), \quad \rho \in \mathfrak{H}_{\mathrm{at}},
$$

for some fixed $V_{\alpha} \in \mathcal{B}\left(\mathbb{C}^{d}\right), \alpha \in \mathrm{A},|\mathrm{A}|<\infty$. Here, $H_{\text {Lamb }}$ is the so-called atomic Lamb shift and $\mathfrak{L}_{d} \in \mathcal{B}\left(\mathfrak{H}_{\text {at }}\right)$ is the effective atomic dissipation. The atom-reservoir interaction yields an effective atomic dynamics generated by $\mathfrak{L}_{\text {at }}+\lambda^{2} \mathfrak{L}_{\mathcal{R}}$ (without pump). Notice furthermore that $H_{\text {Lamb }}$ commutes with the atomic Hamiltonian $H_{\text {at }}$.

Explicit expressions for $H_{\text {Lamb }}$ and $\mathfrak{L}_{d}$ in terms of the microscopic couplings $Q_{\ell_{1}, \ldots, \ell_{\kappa}}^{(\kappa)}$ and $f_{\ell}^{(\kappa)}$ are quite cumbersome in the general case but can straightforwardly be obtained. In the simplest non-trivial case, i.e., if $Q_{\ell_{1}, \ldots, \ell_{\kappa}}^{(\kappa)} \equiv 0$ for $\kappa \geq 2$ and the family of functions (form factors) $\left\{f_{\ell}\right\}_{\ell=1}^{m} \equiv\left\{f_{\ell}^{(1)}\right\}_{\ell=1}^{m} \subset \mathfrak{h}_{1}$ is orthogonal, we obtain the following: Let $\left\{\tilde{V}_{j, k}^{(\ell)}\right\}_{j, k, \ell} \subset \mathcal{B}\left(\mathbb{C}^{d}\right)$ be the family of operators defined by

$$
\tilde{V}_{j, k}^{(\ell)}:=\mathbf{1}\left[H_{\mathrm{at}}=E_{j}\right] Q_{\ell} \mathbf{1}\left[H_{\mathrm{at}}=E_{k}\right]
$$

for $j, k \in\{1,2, \ldots, N\}$ and $\ell \in\{1,2, \ldots, m\}$. Then, the atomic Lamb shift $H_{\text {Lamb }} \in \mathcal{B}\left(\mathbb{C}^{d}\right)$ is the self-adjoint operator defined by

$$
H_{\mathrm{Lamb}}=-\frac{1}{2} \sum_{\epsilon \in \sigma\left(i \mathfrak{L}_{\mathrm{at}}\right) \backslash\{0\}} \sum_{(j, k) \in \mathfrak{t}_{\epsilon}} \sum_{\ell=1}^{m} d_{j, k}^{(\ell)} \tilde{V}_{j, k}^{(\ell) *} \tilde{V}_{j, k}^{(\ell)} .
$$

The real coefficient

$$
d_{j, k}^{(\ell)}:=\mathcal{P} \mathcal{P}\left[f_{\ell}^{(1, \beta)}\left(\cdot+\left(E_{k}-E_{j}\right)\right)\right]
$$

is the principal part $\mathcal{P} \mathcal{P}[f]$ of the function $f \equiv f_{\ell}^{(1, \beta)}\left(\cdot+\left(E_{k}-E_{j}\right)\right)$. See (2.16) for the definition of $f_{\ell}^{(1, \beta)}$. Meanwhile,

$$
\mathfrak{L}_{d}:=\frac{1}{2} \sum_{\epsilon \in \sigma\left(i \mathfrak{L}_{\mathrm{at}}\right)} \sum_{(j, k) \in \mathfrak{t}_{\epsilon}} \sum_{\ell=1}^{m} c_{j, k}^{(\ell)} \mathfrak{L}_{j, k}^{(\ell)}
$$

where $c_{j, k}^{(\ell)}:=\pi f_{\ell}^{(1, \beta)}\left(E_{k}-E_{j}\right) \geq 0$ and

$$
\mathfrak{L}_{j, k}^{(\ell)}(\rho):=2 \tilde{V}_{j, k}^{(\ell)} \rho \tilde{V}_{j, k}^{(\ell) *}-\tilde{V}_{j, k}^{(\ell) *} \tilde{V}_{j, k}^{(\ell)} \rho-\rho \tilde{V}_{j, k}^{(\ell) *} \tilde{V}_{j, k}^{(\ell)}, \quad \rho \in \mathfrak{H}_{\text {at }} .
$$

Note that these expressions for $H_{\text {Lamb }}$ and $\mathfrak{L}_{d}$ appear in the heuristic derivation of the time-dependent Lindbladian given in [1, Section 6.1]. See also [15, 1.3. Remarks.].

To control Rabi oscillations (caused by the optical pump, whose strength is of order $\mathcal{O}(\eta)$ ) via the effective atom-reservoir interaction $\lambda^{2} \mathfrak{L}_{\mathcal{R}}$ we assume:

\section{Assumption 3 (Moderate optical pump)}

For a fixed (but arbitrary) constant $C \in \mathbb{R}^{+}$the couplings $\eta, \lambda \in \mathbb{R}$ are chosen such that $|\eta| \leq C \lambda^{2}$.

Moreover, we impose 0 to be a non-degenerated eigenvalue of $\frac{\eta}{2} \mathfrak{L}_{\mathrm{p}}+\lambda^{2} \mathfrak{L}_{\mathcal{R}}$ with some non-trivial real spectral gap, that is, more precisely,

$$
\max \left\{\operatorname{Re}\{w\} \mid w \in \sigma\left(\frac{\eta}{2} \mathfrak{L}_{\mathrm{p}}+\lambda^{2} \mathfrak{L}_{\mathcal{R}}\right) \backslash\{0\}\right\} \leq-\lambda^{2} C<0
$$

with $C \in \mathbb{R}^{+}$being some fixed constant not depending on $\lambda$ and $\eta$. This allows the study of the dynamics of the atom at large times. By the results of [16], the following assumption on the dissipative part $\mathfrak{L}_{d}$ of $\mathfrak{L}_{\mathcal{R}}$ suffices to ensure the above spectral property: 


\section{Assumption 4 (Irreducibility of quantum Markov chains)}

The family $\left\{V_{\alpha}\right\}_{\alpha \in \mathrm{A}} \subset \mathcal{B}\left(\mathbb{C}^{d}\right)$ satisfies

$$
\left(\bigcup_{\alpha \in \mathrm{A}}\left\{V_{\alpha}\right\}\right)^{\prime \prime}=\mathcal{B}\left(\mathbb{C}^{d}\right)
$$

with $M^{\prime \prime}$ being the bicommutant of $M \subset \mathcal{B}\left(\mathbb{C}^{d}\right)$.

See the proof of [1, Lemma 6.3] for the detailed arguments. This last assumption highlights the role played by dissipative effects of the reservoir on the atom in order to get an appropriate asymptotic evolution of populations of atomic levels. For further discussions, see [1, Section 3.2].

From now on, we assume Assumptions 10 to be satisfied.

\section{Microscopic Dynamics}

Let

$$
\delta_{t}^{(\lambda, \eta)}:=\delta+\eta \cos (\varpi t) \delta_{\mathrm{at}, \mathrm{p}}+\lambda \delta_{\mathrm{at}, \mathcal{R}}, \quad t \in \mathbb{R},
$$

with $\lambda, \eta \in \mathbb{R}$ and

$$
\delta_{\mathrm{at}, \mathrm{p}}:=i\left[H_{\mathrm{p}} \otimes \mathbf{1}_{\mathcal{V}_{\mathcal{R}}}, \cdot\right] .
$$

Recall that the generator of the group $\tau:=\left\{\tau_{t}\right\}_{t \in \mathbb{R}}(2.11)$ is the symmetric derivation $\delta$, while $\delta_{\text {at, } \mathcal{R}}$ is defined by (2.17). Therefore, the time-dependent symmetric derivation $\delta_{t}^{(\lambda, \eta)}$ corresponds to a bounded and smooth (w.r.t. $t \in \mathbb{R}$ ) perturbation of $\delta$. For all $\lambda, \eta \in \mathbb{R}$, the (non-autonomous full) microscopic dynamics is defined by the unique strongly continuous two-parameter family $\left\{\tau_{t, s}^{(\lambda, \eta)}\right\}_{t \geq s}$ of $*$-automorphisms of $\mathcal{V}$ satisfying the evolution equation

$$
\forall s, t \in \mathbb{R}, t \geq s: \quad \partial_{t} \tau_{t, s}^{(\lambda, \eta)}=\tau_{t, s}^{(\lambda, \eta)} \circ \delta_{t}^{(\lambda, \eta)}, \quad \tau_{s, s}^{(\lambda, \eta)}:=\mathbf{1}_{\mathcal{V}},
$$

on the (time-constant) domain of $\delta_{t}^{(\lambda, \eta)}$. The existence, uniqueness and strong continuity of the two-parameter family $\left\{\tau_{t, s}^{(\lambda, \eta)}\right\}_{t \geq s}$ is shown by standard arguments, see Proposition 4.1 and Definition 4.2 ,

The time-evolving state of the compound system is then given by

$$
\omega_{t}:=\omega_{0} \circ \tau_{t, 0}^{(\lambda, \eta)}=\left(\omega_{\mathrm{at}} \otimes \omega_{\mathcal{R}}\right) \circ \tau_{t, 0}^{(\lambda, \eta)}, \quad t \in \mathbb{R}_{0}^{+} .
$$

The restriction of this state to the atomic degrees of freedom yields a time-dependent atomic state defined by

$$
\omega_{\text {at }}(t)(A):=\omega_{t}\left(A \otimes \mathbf{1}_{\mathcal{V}_{\mathcal{R}}}\right), \quad A \in \mathcal{B}\left(\mathbb{C}^{d}\right),
$$

for all $t \in \mathbb{R}_{0}^{+}$. The corresponding family of density matrices of $\left\{\omega_{\text {at }}(t)\right\}_{t \in \mathbb{R}_{0}^{+}}$is denoted by $\left\{\rho_{\text {at }}(t)\right\}_{t \in \mathbb{R}_{0}^{+}}$.

The aim of this paper is to prove [1, Theorem 3.3]. This amounts to study the orthogonal projection $P_{\mathfrak{D}}\left(\rho_{\text {at }}(t)\right)$ of the atomic density matrix $\rho_{\text {at }}(t)$ on the subspace

$$
\mathfrak{D} \equiv \mathfrak{D}\left(H_{\text {at }}\right):=\mathcal{B}\left(\mathcal{H}_{1}\right) \oplus \cdots \oplus \mathcal{B}\left(\mathcal{H}_{\mathrm{N}}\right) \subset \mathfrak{H}_{\text {at }}
$$

of block-diagonal matrices. In other words, we analyze the density matrix

$$
P_{\mathfrak{D}}\left(\rho_{\mathrm{at}}(t)\right)=\sum_{k=1}^{N} \mathbf{1}\left[H_{\mathrm{at}}=E_{k}\right] \rho_{\mathrm{at}}(t) \mathbf{1}\left[H_{\mathrm{at}}=E_{k}\right]
$$

for any $t \in \mathbb{R}_{0}^{+}$. In fact, we compare the above time-evolving density matrix with the unique solution of the following (effective) atomic master equation on $\mathfrak{H}_{\text {at }}$ :

$$
\forall t \in \mathbb{R}_{0}^{+}: \quad \frac{\mathrm{d}}{\mathrm{d} t} \rho(t)=\mathfrak{L}_{t}^{(\lambda, \eta)}(\rho(t)), \quad \rho(0)=\rho_{\text {at }}(0) \equiv \rho_{\text {at }} \in \mathfrak{H}_{\text {at }} .
$$

Here, for any $\lambda, \eta, t \in \mathbb{R}, \mathfrak{L}_{t}^{(\lambda, \eta)}$ is a time-dependent Lindbladian defined on $\mathfrak{H}_{\text {at }}$ by

$$
\mathfrak{L}_{t}^{(\lambda, \eta)}:=\mathfrak{L}_{\mathrm{at}}+\eta \cos (\varpi t) \mathfrak{L}_{\mathrm{p}}+\lambda^{2} \mathfrak{L}_{\mathcal{R}},
$$


see (2.8), (2.13) and (2.20)-(2.21).

Since the Lindbladian $\mathfrak{L}_{t}^{(\lambda, \eta)}$ is continuous in time and generates a Markov completely positive (CP) semigroup at any fixed $t \in \mathbb{R}$, there is a continuous two-parameter family denoted by $\left\{\hat{\tau}_{t, s}^{(\lambda, \eta)}\right\}_{t \geq s} \subset \mathcal{B}\left(\mathfrak{H}_{\text {at }}\right)$ of CP tracepreserving maps satisfying the evolution equation:

$$
\forall s, t \in \mathbb{R}, t \geq s: \quad \partial_{t} \hat{\tau}_{t, s}^{(\lambda, \eta)}=\mathfrak{L}_{t}^{(\lambda, \eta)} \circ \hat{\tau}_{t, s}^{(\lambda, \eta)}, \quad \hat{\tau}_{s, s}^{(\lambda, \eta)}:=\mathbf{1}_{\mathfrak{H}_{\mathrm{at}}} .
$$

For any initial condition $\rho_{\text {at }} \in \mathfrak{H}_{\text {at }}$ on has:

$$
\rho(t)=\hat{\tau}_{t, 0}^{(\lambda, \eta)}\left(\rho_{\text {at }}\right), \quad t \in \mathbb{R}_{0}^{+} .
$$

The evolution of the true density matrix $\rho_{\text {at }}(t)$ of the atom at time $t$ depends on the (infinite) degrees of freedom of the reservoir. By contrast, the time evolution of $\rho(t)$ only involves atomic degrees of freedom and hence the effective density matrix $\rho(t)$ evolves in a space of finite dimension. The main interest of the initial value problem (3.6) is that - at small couplings - its solution $\rho(t)$ accurately approximates, for all $t \in \mathbb{R}_{0}^{+}$, the density matrix $\rho_{\text {at }}(t)$ of the time-dependent state $\omega_{\text {at }}(t)$ on the subspace $\mathfrak{D}$ (攵.4), space of populations of the atomic energy levels):

Theorem 3.1 (Validity of the effective atomic master equation)

Assume that $\rho_{\text {at }} \in \mathfrak{D}$ and let $\varepsilon \in(0,1)$. The unique solution $\{\rho(t)\}_{t \geq 0}$ of the effective atomic master equation (3.6) and the atomic density matrix $\left\{\rho_{\text {at }}(t)\right\}_{t \geq 0}$ satisfy the bound

$$
\left\|P_{\mathfrak{D}}\left(\rho_{\text {at }}(t)-\rho(t)\right)\right\|_{\mathfrak{H}_{\text {at }}} \leq C_{\varpi, \varepsilon}|\lambda|^{1-\varepsilon}
$$

for some constant $C_{\varpi, \varepsilon} \in \mathbb{R}^{+}$depending on $\varpi, \varepsilon$, but not on the initial state $\omega_{\text {at }}$ of the atom and the parameters $t, \lambda$, and $\eta$, provided $|\lambda|$ is sufficiently small.

Note that [1, Theorem 3.3] asserts the above bound with $\varepsilon=0$ but for the special case $K=1$. We prove here the slightly weaker bound with $\varepsilon \in(0,1)$, for technical simplicity. [To get the bound for $\varepsilon=0$ one may improve the estimate (4.110).]

\section{Technical Proofs}

The remaining part of the paper is devoted to the proof of Theorem 3.1, which is concluded in Section 4.7 We essentially follow [15, where the notion of $C$-Liouvilleans has been first introduced. Like in [13, 17, 18, the setting of [15] is extended here to allow time-dependent $C$-Liouvilleans.

\subsection{Existence of the non-autonomous dynamics}

Recall that the generator of the group $\tau:=\left\{\tau_{t}\right\}_{t \in \mathbb{R}}$ (2.11) is the symmetric derivation $\delta$. Then, any timedependent and self-adjoint family $\left\{W_{t}\right\}_{t \in \mathbb{R}} \subset \mathcal{V}$ defines a family of symmetric derivations

$$
\delta_{W_{t}}:=\delta+i\left[W_{t}, \cdot\right], \quad t \in \mathbb{R} .
$$

If $\left\{W_{t}\right\}_{t \in \mathbb{R}} \in C^{1}(\mathbb{R}, \mathcal{V})$, then such time-dependent derivations generate a unique fundamental solution $\left\{\mathfrak{W}_{s, t}\right\}_{s, t \in \mathbb{R}}$, which is in our case a family of $*$-automorphisms of $\mathcal{V}$. By fundamental solution, we mean here that the family $\left\{\mathfrak{W}_{s, t}\right\}_{s, t \in \mathbb{R}}$ of bounded operators acting on $\mathcal{V}$ is strongly continuous, preserves the domain

$$
\operatorname{Dom}\left(\delta_{W_{t}}\right)=\operatorname{Dom}(\delta),
$$

satisfies

$$
\begin{aligned}
& \mathfrak{W}_{\cdot, t}(A) \in C^{1}\left(\mathbb{R} ;\left(\operatorname{Dom}(\delta),\|\cdot\|_{\mathcal{V}}\right)\right), \\
& \mathfrak{W}_{s, \cdot}(A) \in C^{1}\left(\mathbb{R} ;\left(\operatorname{Dom}(\delta),\|\cdot\|_{\mathcal{V}}\right)\right),
\end{aligned}
$$

for all $A \in \operatorname{Dom}(\delta)$, and solves the corresponding Cauchy initial value problem

$$
\forall s, t \in \mathbb{R}: \quad \partial_{s} \mathfrak{W}_{s, t}=-\delta_{W_{s}} \circ \mathfrak{W}_{s, t}, \quad \mathfrak{W}_{t, t}=\mathbf{1}_{\mathcal{V}},
$$

in the strong sense on $\left(\operatorname{Dom}(\delta),\|\cdot\|_{\mathcal{V}}\right)$ : 
Proposition 4.1 (Non-autonomous $C^{*}$-dynamics-I)

If $\left\{W_{t}\right\}_{t \in \mathbb{R}} \in C^{1}(\mathbb{R}, \mathcal{V})$, then there is a unique evolution family $\left\{\mathfrak{W}_{s, t}\right\}_{s, t \in \mathbb{R}}$ of $*$-automorphisms with the following properties:

(i) It satisfies the property

$$
\forall t, r, s \in \mathbb{R}: \quad \mathfrak{W}_{s, t}=\mathfrak{W}_{s, r} \mathfrak{W}_{r, t} .
$$

(ii) It is the fundamental solution of (4.2).

(iii) It solves in the strong sense on $\left(\overline{\operatorname{Dom}}(\delta),\|\cdot\|_{\mathcal{V}}\right)$ the abstract Cauchy initial value problem

$$
\forall s, t \in \mathbb{R}: \quad \partial_{t} \mathfrak{W}_{s, t}=\mathfrak{W}_{s, t} \circ \delta_{W_{t}}, \quad \mathfrak{W}_{s, s}=\mathbf{1}_{\mathcal{V}} .
$$

(iv) If $\left\{W_{t}\right\}_{t \in \mathbb{R}}$ is periodic with period $T>0$, then

$$
\forall s, t \in \mathbb{R}, p \in \mathbb{Z}: \quad \mathfrak{W}_{s, t}=\mathfrak{W}_{s+p T, t+p T} .
$$

Proof. The proof of Assertions (i)-(iii) can be done by standard arguments as $\left\{W_{t}\right\}_{t \in \mathbb{R}} \subset \mathcal{V}$. We recommend the proof of [22, Proposition 5.4] where we additionally show [22, Eq. (5.24)], that is,

$$
\mathfrak{W}_{s, t}(A):=\tau_{-s}\left(\mathfrak{V}_{t, s} \tau_{t}(A) \mathfrak{V}_{t, s}^{*}\right)
$$

for any $A \in \mathcal{V}$ and $s, t \in \mathbb{R}$. Here, $\mathfrak{V}_{t, s} \in \mathcal{V}$ is given by the absolutely convergent series

$$
\mathfrak{V}_{t, s}:=\mathbf{1}_{\mathcal{V}}+\sum_{k \in \mathbb{N}} i^{k} \int_{s}^{t} \mathrm{~d} s_{1} \cdots \int_{s}^{s_{k-1}} \mathrm{~d} s_{k} \tau_{s_{k}}\left(W_{s_{k}}\right) \cdots \tau_{s_{1}}\left(W_{s_{1}}\right)
$$

in the Banach space $\left(\operatorname{Dom}(\delta),\|\cdot\|_{\operatorname{Dom}(\delta)}\right)$, where $\|\cdot\|_{\operatorname{Dom}(\delta)}$ stands for the graph norm of the closed operator $\delta$. In particular, if $\left\{W_{t}\right\}_{t \in \mathbb{R}}$ is also periodic with period $T>0, \tau_{-p T}\left(\mathfrak{V}_{t+p T, s+p T}\right)=\mathfrak{V}_{t, s}$ and we obtain Assertion (iv) by using Equation (4.3).

Then, the microscopic dynamics (3.2) corresponds to the following definition:

\section{Definition 4.2 (Non-autonomous $C^{*}$-dynamics-II)}

The non-autonomous dynamics $\left\{\tau_{t, s}\right\}_{t \geq s}$ is defined by $\tau_{t, s}:=\mathfrak{W}_{s, t}$ for $t \geq s$, provided $\left\{W_{t}\right\}_{t \in \mathbb{R}} \in C^{1}(\mathbb{R}, \mathcal{V})$.

Indeed, by construction, this family is the (unique) solution of the abstract Cauchy initial value problem

$$
\forall s, t \in \mathbb{R}: \quad \partial_{t} \tau_{t, s}=\tau_{t, s} \circ \delta_{W_{t}}, \quad \tau_{s, s}=\mathbf{1}_{\mathcal{V}},
$$

in the strong sense on $\left(\operatorname{Dom}(\delta),\|\cdot\|_{\mathcal{V}}\right)$.

\subsection{Time-dependent $C$-Liouvilleans 1}

Assume first that the initial state $\omega_{0}(2.12)$ is of the form

$$
\omega_{0}=\mathfrak{g}_{\mathrm{at}} \otimes \omega_{\mathcal{R}}
$$

where $\mathfrak{g}_{\text {at }}$ is the (Gibbs) state corresponding to the density matrix $\rho_{\mathfrak{g}}(2.3)$. Denote by $\left(\mathfrak{H}_{\text {at }}, \pi_{\mathrm{at}}, \Omega_{\mathrm{at}, \mathfrak{g}}\right)$ and $\left(\mathfrak{H}_{\mathcal{R}}, \pi_{\mathcal{R}}, \Omega_{\mathcal{R}}\right)$ GNS representations of respectively $\mathfrak{g}_{\text {at }}$ and $\omega_{\mathcal{R}}$. Let $\mathfrak{H}:=\mathfrak{H}_{\text {at }} \otimes \mathfrak{H}_{\mathcal{R}}, \pi:=\pi_{\text {at }} \otimes \pi_{\mathcal{R}}$ and $\Omega_{\mathfrak{g}}:=\Omega_{\mathrm{at}, \mathfrak{g}} \otimes \Omega_{\mathcal{R}}$. Observe $\Omega_{\mathfrak{g}}$ is cyclic because it is the tensor product of cyclic vectors and hence, $\left(\mathfrak{H}, \pi, \Omega_{\mathfrak{g}}\right)$ is a GNS representation of $\omega_{0}$. An important property of the initial state $\omega_{0}$ is that it is faithful. In particular, $\pi$ is injective. So, to simplify notation, $\pi(A)$ and $\pi(\mathcal{V})$ are identified with $A$ and $\mathcal{V}$, respectively. The weak closure of the $C^{*}$-algebra $\mathcal{V} \subset \mathcal{B}(\mathfrak{H})$ is the von Neumann algebra $\mathcal{V}^{\prime \prime}$ denoted by $\mathfrak{M}$. The state $\omega_{0}$ is a $(\tau, \beta)-\mathrm{KMS}$ state, where $\left\{\tau_{t}\right\}_{t \in \mathbb{R}}$ is the one-parameter group of $*$-automorphisms on $\mathcal{V}$ defined by (2.11). By [20, Corollary 5.3.9], the cyclic vector $\Omega_{\mathfrak{g}}$ is separating for $\mathfrak{M}$, i.e., $A \Omega_{\mathfrak{g}}=0$ implies $A=0$ for all $A \in \mathfrak{M}$. The state $\omega_{0}$ on $\mathcal{V}$ extends uniquely to a normal state on the von Neumann algebra $\mathfrak{M}$ and $\left\{\tau_{t}\right\}_{t \in \mathbb{R}}$ uniquely extends to a $\sigma$-weakly continuous $*$-automorphism group on $\mathfrak{M}$, see [20, Corollary 5.3.4]. Both extensions are again denoted

\footnotetext{
${ }^{1}$ This section has been partially done in collaboration with M. Westrich during his $\mathrm{PhD}$ [19, Chapter 5].
} 
by $\omega_{0}$ and $\left\{\tau_{t}\right\}_{t \in \mathbb{R}}$, respectively. Because $\omega_{0}$ is invariant w.r.t. $\left\{\tau_{t}\right\}_{t \in \mathbb{R}}$, there is a unique unitary representation $\left\{U_{t}\right\}_{t \in \mathbb{R}}$ of $\left\{\tau_{t}\right\}_{t \in \mathbb{R}}$ by conjugation, i.e.,

$$
\forall t \in \mathbb{R}, A \in \mathfrak{M}: \quad \tau_{t}(A)=U_{t} A U_{t}^{*},
$$

such that $U_{t} \Omega_{\mathfrak{g}}=\Omega_{\mathfrak{g}}$. As $t \mapsto \tau_{t}$ is $\sigma$-weakly continuous, the map $t \mapsto U_{t}$ is strongly continuous. Therefore, there is a a self-adjoint operator $L_{\mathfrak{g}}$ with $U_{t}=\mathrm{e}^{i t L_{\mathfrak{g}}}$ for all $t \in \mathbb{R}$. In particular, $\Omega_{\mathfrak{g}} \in \operatorname{Dom}\left(L_{\mathfrak{g}}\right)$ and $L_{\mathfrak{g}}$ annihilates $\Omega_{\mathfrak{g}}$, i.e.,

$$
L_{\mathfrak{g}} \Omega_{\mathfrak{g}}=0 .
$$

Moreover, $L_{\mathfrak{g}}$ is related to the generator $\delta$ of the group $\left\{\tau_{t}\right\}_{t \in \mathbb{R}}$ by the following relations:

$$
\left\{A \Omega_{\mathfrak{g}}: A \in \operatorname{Dom}(\delta)\right\} \subset \operatorname{Dom}\left(L_{\mathfrak{g}}\right) \subset \mathfrak{H}
$$

and

$$
\forall A \in \operatorname{Dom}(\delta): \quad L_{\mathfrak{g}}\left(A \Omega_{\mathfrak{g}}\right)=\delta(A) \Omega_{\mathfrak{g}} .
$$

The (Tomita-Takesaki) modular objects of the pair $\left(\mathfrak{M}, \Omega_{\mathfrak{g}}\right)$ are important for our further analysis. We write $\Delta_{\mathfrak{g}}=\mathrm{e}^{-\beta L_{\mathfrak{g}}}, J_{\mathfrak{g}}$, and

$$
\mathcal{P}_{\mathfrak{g}}:=\overline{\left\{A J_{\mathfrak{g}} A J_{\mathfrak{g}} \Omega_{\mathfrak{g}}: A \in \mathfrak{M}\right\}}
$$

respectively for the modular operator, the modular conjugation and the natural positive cone of the pair $\left(\mathfrak{M}, \Omega_{\mathfrak{g}}\right)$. For a detailed exposition on the use of the Tomita-Takesaki modular theory in quantum statistical mechanics, see, for instance, the textbook 21. The family $\left\{U_{t}\right\}_{t \in \mathbb{R}}$ of unitaries representing $\left\{\tau_{t}\right\}_{t \in \mathbb{R}}$ by conjugation also satisfies $U_{t} \mathcal{P}_{\mathfrak{g}} \subset \mathcal{P}_{\mathfrak{g}}$ for any $t \in \mathbb{R}$. The self-adjoint operator $L_{\mathfrak{g}}$, generator of the strongly continuous group $\left\{U_{t}\right\}_{t \in \mathbb{R}}$ on $\mathfrak{H}$, is named the standard Liouvillean of the $*$-automorphism group $\left\{\tau_{t}\right\}_{t \in \mathbb{R}}$.

Now, if the faithful state $\omega_{\text {at }}$ is not the Gibbs state $\mathfrak{g}_{\text {at }}$ in $(2.12)$ then, because $\Omega_{\text {at }}$ is cyclic for $\pi_{\text {at }}\left(\mathcal{B}\left(\mathbb{C}^{d}\right)\right)$, a GNS representation of

$$
\omega_{0}:=\omega_{\mathrm{at}} \otimes \omega_{\mathcal{R}}
$$

is also given by $(\mathfrak{H}, \pi, \Omega)$ where $\Omega=\Omega_{\text {at }} \otimes \Omega_{\mathcal{R}}$ for some $\Omega_{\text {at }} \in \mathfrak{H}_{\text {at }}$, see (2.6).

Observe that $\Omega=A J_{\mathfrak{g}} A J_{\mathfrak{g}} \Omega_{\mathfrak{g}} \in \mathcal{P}_{\mathfrak{g}}$ with

$$
A=\rho_{\text {at }}^{1 / 4} \rho_{\mathfrak{g}}^{-1 / 4} \otimes \mathbf{1}_{\mathfrak{H}_{\mathcal{R}}},
$$

where we recall that $\rho_{\mathfrak{g}}$ is the density matrix (2.3) of the Gibbs state $\mathfrak{g}_{\text {at }}$. Additionally, by cyclicity of $\Omega$ and 21. Proposition 2.5.30 (1)], $\Omega$ is also separating for $\mathfrak{M}$. So, we can define the modular operator $\Delta$ and the modular conjugation $J$ of the pair $(\mathfrak{M}, \Omega)$. By [21, Proposition 2.5.30 (2)], $J_{\mathfrak{g}}=J$.

Assume that the density matrix $\rho_{\text {at }}$ of the initial atomic state is block-diagonal in the eigenbasis of the atomic Hamiltonian $H_{\text {at }}$, i.e., $\rho_{\text {at }} \in \mathfrak{D}$ (cf. (3.4) $)$. Then $\rho_{\text {at }}$ commutes with $H_{\text {at }}$ an it is straightforward to verify that

$$
L_{\mathfrak{g}} \Omega=0 \text {. }
$$

In our setting, however, the free dynamics is perturbed by the pump and the atom-reservoir interaction. Altogether, this leads to a perturbation $W_{t}$ of $L_{\mathfrak{g}}$. For autonomous perturbations of the generator $\delta$ of the dynamics $\left\{\tau_{t}\right\}_{t \in \mathbb{R}}($ on $\mathcal{V})$ of the form $i[W, \cdot]$ with some self-adjoint $W \in \mathcal{V}$, one has

$$
\forall t \in \mathbb{R}, A \in \mathcal{V}: \quad \tau_{t}^{W}(A)=\mathrm{e}^{i t\left(L_{\mathfrak{g}}+W\right)} A \mathrm{e}^{-i t\left(L_{\mathfrak{g}}+W\right)} \in \mathfrak{M},
$$

where $\left\{\tau_{t}^{W}\right\}_{t \in \mathbb{R}}$ is the strongly continuous $*$-automorphism group on $\mathcal{V}$ generated by $\delta+i[W, \cdot]$. Analogously as above, $\left\{\tau_{t}^{W}\right\}_{t \in \mathbb{R}}$ defines a $\sigma$-weakly continuous group on whole $\mathfrak{M}$. Nevertheless, in general, the operator $L_{\mathfrak{g}}+W$ does not annihilate anymore $\Omega$. A way to get around this problem is presented in [15, Section 2.2] by introducing the notion of $C$-Liouvilleans $\mathcal{L}$, which is constructed such that $\mathcal{L} \Omega=0$.

Observe that, in our case, the dynamics is non-autonomous. Using the $C$-Liouvilleans construction of [15, Section 2.2] we can design the time-depending $C$-Liouvillean of the non-autonomous dynamics such that $\mathcal{L}_{t} \Omega=0$. This is a very useful property for the analysis of the dynamics. As already done in a few previous 
works (see for instance [13, 17, 18]), we thus extend the definition of $C$-Liouvilleans [15, Section 2.2] to nonautonomous evolutions. Note that we exploit the fact that the density matrix $\rho_{\text {at }}$ of the initial atomic state commutes 2 with the atomic Hamiltonian $H_{\text {at }}$. This simplifies the explicit expression of the $C$-Liouvillean, see Definition 4.3 and (4.27) below.

Following [15, Section 2.2], we define the linear space

$$
\mathcal{O}:=\{A \Omega: A \in \mathcal{V}\} \subset \mathfrak{H}
$$

Let $\iota$ be the map from $\mathcal{V}$ to $\mathcal{O}$ defined by $\iota(A):=A \Omega$. This map is an isomorphism of the linear spaces $\mathcal{V}$ and $\mathcal{O}$ because $\Omega$ is a separating vector for $\mathfrak{M}$. In particular, $\|A \Omega\|_{\mathcal{O}}:=\|A\|_{\mathcal{V}}$ defines a norm on the space $\mathcal{O}, \iota$ is an isometry w.r.t. this norm, and $\left(\mathcal{O},\|\cdot\|_{\mathcal{O}}\right)$ is a Banach space. Any element $A \in \mathcal{V}$ also defines a bounded operator on $\mathcal{O}$ by left multiplication, i.e., $A(B \Omega):=(A B) \Omega$. Moreover, we define a strongly continuous two-parameter family $\left\{T_{s, t}\right\}_{s, t \in \mathbb{R}}$ acting on $\mathcal{O}$ by

$$
T_{s, t}:=\iota \circ \mathfrak{W}_{s, t} \circ \iota^{-1}, \quad s, t \in \mathbb{R}
$$

Note that, since $\left\{\mathfrak{W}_{s, t}\right\}_{s, t \in \mathbb{R}}$ is a family of $*$-automorphisms, the operator $T_{s, t}$ has a bounded inverse. Moreover,

$$
\forall s, t \in \mathbb{R}, A \in \mathcal{V}: \quad T_{s, t}(\Omega)=\Omega \quad \text { and } \quad T_{s, t} A T_{s, t}^{-1}=\mathfrak{W}_{s, t}(A) .
$$

Here, $A, \mathfrak{W}_{s, t}(A) \in \mathcal{V}$ are seen as bounded operators on $\mathcal{O}$ defined by left multiplication, as explained above. We would like now to extend the two-parameter family $\left\{T_{s, t}\right\}_{s, t \in \mathbb{R}}$ to whole $\mathfrak{H}$. To this end, similarly as done in [15. Eq. (2.5)] for the autonomous case, we define the time-dependent $C$-Liouvillean as follows:

\section{Definition 4.3 (Time-dependent $C$-Liouvilleans)}

For any self-adjoint family $\left\{W_{t}\right\}_{t \in \mathbb{R}} \subset \mathcal{V}$, the time-dependent $C$-Liouvillean is the family of operators acting on $\mathfrak{H}$ defined by

$$
\mathcal{L}_{t}:=L_{\mathfrak{g}}+W_{t}-J \Delta^{1 / 2} W_{t} \Delta^{-1 / 2} J, \quad t \in \mathbb{R}
$$

This time-dependent operator is, in general, not anymore self-adjoint. Note also that the term

$$
V_{t}:=W_{t}-J \Delta^{1 / 2} W_{t} \Delta^{-1 / 2} J \in \mathcal{B}(\mathcal{O})
$$

implements the commutator $\left[W_{t}, \cdot\right]$ on $\mathcal{O}$ for any $t \in \mathbb{R}$. Indeed, for any $A \in \mathcal{V}$,

$$
\left[W_{t}, A\right] \Omega=W_{t} A \Omega-\left(W_{t} A^{*}\right)^{*} \Omega
$$

and using $J \Delta^{1 / 2} A \Omega=A^{*} \Omega, \Delta^{-1 / 2}=J \Delta^{1 / 2} J$, and $J^{2}=1$,

$$
J \Delta^{1 / 2} W_{t} \Delta^{-1 / 2} J A \Omega=\left(W_{t} A^{*}\right)^{*} \Omega
$$

In particular,

$$
\left\|J \Delta^{1 / 2} W_{t} \Delta^{-1 / 2} J\right\|_{\mathcal{B}(\mathcal{O})}=\left\|W_{t}\right\|_{\mathcal{V}}<\infty
$$

and

$$
\forall t \in \mathbb{R}: \quad \mathcal{L}_{t} \Omega=0
$$

Observe that the norm $\|\cdot\|_{\mathcal{O}}$ on $\mathcal{O}$ is not equivalent to the Hilbert space norm on this subspace of $\mathfrak{H}$. In particular, the boundedness of the operator

$$
J \Delta^{1 / 2} W_{t} \Delta^{-1 / 2} J
$$

as an operator on $\mathfrak{H}$ is unclear, in spite of (4.13). Therefore, for every $t \in \mathbb{R}$, we assume some sufficient conditions on the operator family $\left\{V_{t}\right\}_{t \in \mathbb{R}}$, like the boundedness of its elements as linear operators on the Hilbert space $\mathfrak{H}$, in order to extend the elements of the two-parameter family $\left\{T_{s, t}\right\}_{s, t \in \mathbb{R}}$ to whole $\mathfrak{H} \supset \mathcal{O}$.

\footnotetext{
${ }^{2}$ The mp_arc preprint of this paper gives this construction without this assumption.
} 
Proposition 4.4 (Extension of $\left.\left\{T_{s, t}\right\}_{s, t \in \mathbb{R}}-\mathbf{I}\right)$

Assume that $\left\{V_{t}\right\}_{t \in \mathbb{R}} \in C^{1}(\mathbb{R}, \mathcal{B}(\mathfrak{H}))$. Then, there is a unique evolution family $\left\{U_{s, t}\right\}_{s, t \in \mathbb{R}} \subset \mathcal{B}(\mathfrak{H})$ with the following properties:

(i) It satisfies the property

$$
\forall t, r, s \in \mathbb{R}: \quad U_{s, t}=U_{s, r} U_{r, t} .
$$

(ii) It is the unique fundamental solution of the abstract Cauchy initial value problem

$$
\forall s, t \in \mathbb{R}: \quad \partial_{s} U_{s, t}=-i \mathcal{L}_{s} U_{s, t}, \quad U_{t, t}=\mathbf{1}_{\mathfrak{H}} .
$$

(iii) It solves, in the strong sense on $\operatorname{Dom}\left(L_{\mathfrak{g}}\right)$, the Cauchy initial value problem

$$
\forall s, t \in \mathbb{R}: \quad \partial_{t} U_{s, t}=i U_{s, t} \mathcal{L}_{t}, \quad U_{s, s}=\mathbf{1}_{\mathfrak{H}} .
$$

(iv) For any $s, t \in \mathbb{R}, U_{t, s}$ has a bounded inverse $U_{t, s}^{-1}$.

(v) If $\left\{W_{t}\right\}_{t \in \mathbb{R}}$ is periodic with period $T>0$, then

$$
\forall s, t \in \mathbb{R}, p \in \mathbb{Z}: \quad U_{s, t}=U_{s+p T, t+p T} .
$$

Proof. This result corresponds to [13, Lemma 3.1]. The arguments of its proof are standard and thus only partially indicated in [13. We prove the proposition here for completeness. Since $\mathcal{L}_{t}=L_{\mathfrak{g}}+V_{t}$, Assertions (i)-(iii) can easily be deduced from [23, Sect. 5.4, Theorem 4.8.]. To prove that $U_{t, s}$ has a bounded inverse $U_{s, t}^{-1}$, it suffices to prove that $U_{s, t}^{-1} \in \mathcal{B}(\mathfrak{H})$ exists for small time differences $|t-s|$, by the property (i). To this aim, we observe that

$$
\forall s, t \in \mathbb{R}: \quad U_{s, t}=\mathrm{e}^{i(t-s) L_{\mathfrak{g}}}+i \int_{s}^{t} U_{s, r} V_{r} \mathrm{e}^{i(r-t) L_{\mathfrak{g}}} \mathrm{d} r,
$$

in the strong sense. See, e.g., 23. Sect. 5.4, Lemma 4.5. and Theorem 4.6.]. Using that $\left\{V_{t}\right\}_{t \in \mathbb{R}} \in C^{1}(\mathbb{R}, \mathcal{B}(\mathfrak{H}))$, it follows by standard arguments that $\left\|U_{r, r^{\prime}}\right\|_{\mathcal{B}(\mathfrak{H})}$ is uniformly bounded for $r, r^{\prime}$ in compact subsets of $\mathbb{R}$. [To show this, $U_{s, t}$ can, for instance, be represented by Dyson-Phillips series.] Therefore, by unitarity of $\mathrm{e}^{i(t-s) L_{\mathfrak{g}}}$, for any $t \in \mathbb{R}$ and sufficiently small $|t-s|$ one has

$$
\left\|\int_{s}^{t} U_{s, r} V_{r} \mathrm{e}^{i(r-t) L_{\mathfrak{g}}} \mathrm{d} r\right\|_{\mathcal{B}(\mathfrak{H})} \leq \frac{1}{2}
$$

By using Neumann series to construct $U_{s, t}^{-1} \in \mathcal{B}(\mathfrak{H})$ for such times, Assertion (iv) holds for all $s, t \in \mathbb{R}$ because of (i). If $\left\{W_{t}\right\}_{t \in \mathbb{R}}$ is $T$-periodic, then both $\left\{U_{s, t}\right\}_{s, t \in \mathbb{R}}$ and $\left\{U_{s+p T, t+p T}\right\}_{s, t \in \mathbb{R}}$ satisfy the integral equation (4.15). By uniqueness of solution of (4.15) (cf. 23, Sect. 5.4, Lemma 4.5.]), it follows that $\left\{U_{s, t}\right\}_{s, t \in \mathbb{R}}$ is $T$-periodic in this case.

Combining this with Proposition 4.1 we deduce that the evolution family $\left\{U_{s, t}\right\}_{s, t \in \mathbb{R}}$ is the unique continuous extension of the two-parameter family $\left\{T_{s, t}\right\}_{s, t \in \mathbb{R}}$ to the Hilbert space $\mathfrak{H}$ :

Proposition 4.5 (Extension of $\left\{T_{s, t}\right\}_{s, t \in \mathbb{R}^{-}} \mathbf{I I}$ )

Assume that $\left\{W_{t}\right\}_{t \in \mathbb{R}} \in C^{1}(\mathbb{R}, \mathcal{V})$ and $\left\{V_{t}\right\}_{t \in \mathbb{R}} \in C^{1}(\mathbb{R}, \mathcal{B}(\mathfrak{H}))$. Then, the evolution family of Proposition 4.4 satisfies $U_{s, t} \Omega=\Omega$ and

$$
\forall s, t \in \mathbb{R}, A \in \mathcal{V}: \quad \mathfrak{W}_{s, t}(A)=U_{s, t} A U_{s, t}^{-1} .
$$

Here, $A \equiv \pi(A)$ and $\mathfrak{W}_{s, t}(A) \equiv \pi\left(\mathfrak{W}_{s, t}(A)\right)$ are seen as bounded operators on $\mathfrak{H}$, by left multiplication.

Proof. This proposition corresponds to [13, Theorem 3.2]. Here we propose an alternative proof which is somewhat simpler. Note first that $U_{s, t} \Omega=\Omega$ is a direct consequence of (4.14) and Proposition 4.4 (iii). Using the isometry between $\mathcal{O}$ and $\mathcal{V}$,

$$
T_{\cdot, t}(A \Omega) \in C^{1}(\mathbb{R} ; \mathcal{O}), \quad T_{s, .}(A \Omega) \in C^{1}(\mathbb{R} ; \mathcal{O})
$$


for any $A \in \operatorname{Dom}(\delta)$ and $s, t \in \mathbb{R}$, by Proposition 4.1 (ii). By using Proposition 4.1 (ii)-(iii), (4.1), (4.6)-(4.7) with $\Omega$ replacing $\Omega_{\mathfrak{g}}$, (4.9), and (4.11)-(4.12), it follows that

$$
\begin{aligned}
& \partial_{s} T_{s, t}(A \Omega)=-\delta_{W_{s}}\left(\mathfrak{W}_{s, t}(A)\right) \Omega=-i \mathcal{L}_{s}\left(T_{s, t}(A \Omega)\right) \\
& \partial_{t} T_{s, t}(A \Omega)=\mathfrak{W}_{s, t}\left(\delta_{W_{t}}(A)\right) \Omega=i T_{s, t}\left(\mathcal{L}_{t}(A \Omega)\right)
\end{aligned}
$$

for all $s, t \in \mathbb{R}$ and $A \in \operatorname{Dom}(\delta)$. Meanwhile, since

$$
\forall A \in \mathcal{V}: \quad\|A \Omega\|_{\mathfrak{H}} \leq\|A\|_{\mathcal{V}}=\|A \Omega\|_{\mathcal{O}},
$$

we have

$$
T_{\cdot, t}(A \Omega) \in C^{1}\left(\mathbb{R} ;\left(\operatorname{Dom}\left(L_{\mathfrak{g}}\right),\|\cdot\|_{\mathfrak{H}}\right)\right), \quad T_{s, \cdot}(A \Omega) \in C^{1}\left(\mathbb{R} ;\left(\operatorname{Dom}\left(L_{\mathfrak{g}}\right),\|\cdot\|_{\mathfrak{H}}\right)\right),
$$

and (4.16)-(4.17) also holds in the sense of $\mathfrak{H}$. By Proposition 4.4 (ii),

$$
\forall A \in \operatorname{Dom}(\delta): \quad U_{s, t}(A \Omega)=T_{s, t}(A \Omega):=\mathfrak{W}_{s, t}(A) \Omega .
$$

By density of $\operatorname{Dom}(\delta)$ in $\mathcal{V}$, for any $A \in \mathcal{V}$, there is a sequence $\left\{A_{n}\right\}_{n=1}^{\infty} \subset \operatorname{Dom}(\delta)$ converging in $\mathcal{V}$ to $A$. We infer from (4.18) that this sequence $\left\{A_{n}\right\}_{n=1}^{\infty}$ also converges to $A$ in the sense of $\mathfrak{H}$. On the one hand, by the boundedness of $U_{s, t}$ in $\mathfrak{H}$,

$$
\lim _{n \rightarrow \infty} U_{s, t}\left(A_{n} \Omega\right)=U_{s, t}(A \Omega) .
$$

On the other hand, using (4.19) and the boundedness of $T_{t, s}$ in $\mathcal{O}$, one gets

$$
\lim _{n \rightarrow \infty} U_{s, t}\left(A_{n} \Omega\right)=\lim _{n \rightarrow \infty} T_{s, t}\left(A_{n} \Omega\right)=T_{s, t}(A \Omega) .
$$

As a consequence, $\left.U_{s, t}\right|_{\mathcal{O}}=T_{s, t}$. In particular, from the uniqueness of the inverse, one has $\left.U_{s, t}^{-1}\right|_{\mathcal{O}}=T_{s, t}^{-1}$. We then use (4.10) to deduce that

$$
\forall s, t \in \mathbb{R}, A \in \mathcal{V}, x \in \mathcal{O}: \mathfrak{W}_{s, t}(A) x=U_{s, t} A U_{s, t}^{-1}(x)
$$

By density of $\mathcal{O}$ in $\mathfrak{H}$, we arrive at the assertion.

The use of $C$-Liouvilleans is advantageous because of the following identity:

$$
\forall s, t \in \mathbb{R}, A \in \mathfrak{M}: \quad U_{s, t} A U_{s, t}^{-1} \Omega=U_{s, t} A \Omega .
$$

\subsection{Dynamics in an explicit GNS representation}

To obtain the atomic Lindbladians (3.7) from the time-dependant $C$-Liouvilleans (Definition 4.3), we use a convenient explicit GNS representation of the initial state (2.12). As already mentioned, this GNS representation includes the GNS representation defined in Section 2.2 for the atomic initial state $\omega_{\text {at. }}$ So, it remains to give an explicit GNS representation $\left(\mathfrak{H}_{\mathcal{R}}, \pi_{\mathcal{R}}, \Omega_{\mathcal{R}}\right)$ for the $\left(\tau^{\mathcal{R}}, \beta\right)$-KMS state $\omega_{\mathcal{R}}$ of the fermionic reservoir at inverse temperature $\beta \in \mathbb{R}^{+}$. As briefly discussed in [1, Section 6.1], we use the so-called Jakšić-Pillet glued representation [15, because it is well-adapted to the application of spectral deformation methods, see Section 4.5 .

Consider the Hilbert space

$$
\mathfrak{h}_{2}:=L^{2}\left(\mathbb{R} \times S^{2}, \mathbb{C}\right),
$$

where $S^{2} \subset \mathbb{R}^{3}$ is the two-dimensional unit sphere centered at the origin and $\mathbb{R} \times S^{2}$ (spherical coordinates of $\mathbb{R}^{3} \times \mathbb{R}^{3}$ ) is equipped with the measure $\mathrm{d} \lambda \otimes \mathrm{d}^{2} s$. Here, $\mathrm{d}^{2} s$ is the usual rotation invariant measure induced by the Euclidean norm of $\mathbb{R}^{3}$ on $S^{2}$ and $\mathrm{d} \lambda$ is the Lebesgue measure. The Hilbert space of the Jakšić-Pillet glued representation is the antisymmetric Fock space

$$
\mathfrak{H}_{\mathcal{R}}:=\mathcal{F}_{-}\left(\mathfrak{h}_{2}\right)
$$


The cyclic vector $\Omega_{\mathcal{R}}$ is the vacuum of $\mathcal{F}_{-}\left(\mathfrak{h}_{2}\right)$. The representation map $\pi_{\mathcal{R}}$ of the $C^{*}$-algebra $\mathcal{V}_{\mathcal{R}}$ is the $C^{*}$-homomorphism uniquely defined by

$$
\forall f \in \mathfrak{h}_{1}: \quad \pi_{\mathcal{R}}(\Phi(f))=\tilde{\Phi}\left(g_{f}\right),
$$

with $\Phi, \tilde{\Phi}$ being the field operators defined by (2.18) respectively on $\mathcal{F}_{-}\left(\mathfrak{h}_{1}\right)$ and $\mathcal{F}_{-}\left(\mathfrak{h}_{2}\right)$, and where $g_{f} \in \mathfrak{h}_{2}$ is given, for $(p, \vartheta) \in \mathbb{R} \times S^{2}$ a.e., by

$$
g_{f}(p, \vartheta):=|p|\left(1+\mathrm{e}^{-\beta p}\right)^{-1 / 2} \begin{cases}f(p \vartheta) & , \quad p \geq 0, \\ \overline{f(-p \vartheta)}, & p<0 .\end{cases}
$$

Compare with (2.14).

Note that $\omega_{\mathcal{R}}$ is faithful and we thus identify $\pi_{\mathcal{R}}(A)$ and $\pi_{\mathcal{R}}\left(\mathcal{V}_{\mathcal{R}}\right)$ with $A$ and $\mathcal{V}_{\mathcal{R}}$, respectively. The weak closure of the $C^{*}$-algebra $\pi_{\mathcal{R}}\left(\mathcal{V}_{\mathcal{R}}\right) \equiv \mathcal{V}_{\mathcal{R}}$ is the von Neumann algebra $\mathfrak{M}_{\mathcal{R}}:=\mathcal{V}_{\mathcal{R}}^{\prime \prime}$. The family $\left\{\tau_{t}^{\mathcal{R}}\right\}_{t \in \mathbb{R}}$ of Bogoliubov automorphisms on the algebra $\mathcal{V}_{\mathcal{R}}$ analogously defined as in (2.1) uniquely extends to an automorphism group of the von Neumann algebra $\mathfrak{M}_{\mathcal{R}}$, again denoted by $\tau^{\mathcal{R}}:=\left\{\tau_{t}^{\mathcal{R}}\right\}_{t \in \mathbb{R}} .\left(\mathfrak{M}_{\mathcal{R}}, \tau^{\mathcal{R}}\right)$ defines a $W^{*}$-dynamical system and $\omega_{\mathcal{R}}$ is a $\left(\tau^{\mathcal{R}}, \beta\right)-\mathrm{KMS}$ state. Hence, $\Omega_{\mathcal{R}}$ is cyclic and separating for $\mathfrak{M}_{\mathcal{R}}$.

As above, there is a unique unitary representation of $\tau^{\mathcal{R}}$ by conjugation, the generator $i L_{\mathcal{R}}$ of which satisfies $L_{\mathcal{R}} \Omega_{\mathcal{R}}=0$ and $\tau_{t}^{\mathcal{R}}(\cdot)=\mathrm{e}^{i t L_{\mathfrak{g}}}(\cdot) \mathrm{e}^{-i t L_{\mathfrak{g}}}$ for all $t \in \mathbb{R}$. The standard Liouvillean $L_{\mathcal{R}}$ is the second quantization

$$
L_{\mathcal{R}}=\mathrm{d} \Gamma(p)
$$

of the multiplication operator by $p \in \mathbb{R}$, that is, the operator acting on $\mathfrak{h}_{2}$ as $(p f)(p, \vartheta)=p f(p, \vartheta)$.

An explicit GNS representation of the initial state $\omega_{0}$ of the composite system is now easy to derive. Using indeed the representations $\left(\mathfrak{H}_{\mathrm{at}}, \pi_{\mathrm{at}}, \Omega_{\mathrm{at}}\right)$ (see Section 2.2) and $\left(\mathfrak{H}_{\mathcal{R}}, \pi_{\mathcal{R}}, \Omega_{\mathcal{R}}\right.$ ) of the states $\omega_{\mathrm{at}}$ and $\omega_{\mathcal{R}}$, a GNS representation $(\mathfrak{H}, \pi, \Omega)$ of $\omega_{0}$ is given by

$$
\mathfrak{H}:=\mathfrak{H}_{\text {at }} \otimes \mathfrak{H}_{\mathcal{R}}, \quad \pi:=\pi_{\text {at }} \otimes \pi_{\mathcal{R}}, \quad \Omega:=\Omega_{\text {at }} \otimes \Omega_{\mathcal{R}} .
$$

Since $\mathfrak{H}_{\text {at }}$ is finite dimensional and $\mathcal{V}:=\mathcal{B}\left(\mathbb{C}^{d}\right) \otimes \mathcal{V}_{\mathcal{R}}$, we do not have to specify the meaning of the tensor product. Recall that, for simplicity of notation, $\pi(A)$ and $\pi(\mathcal{V})$ are respectively identified with $A$ and $\mathcal{V}$. $\mathfrak{M}:=\mathcal{V}^{\prime \prime}$ is the weak closure of the $C^{*}$-algebra $\pi(\mathcal{V}) \equiv \mathcal{V}$. In this explicit representation, $\mathfrak{M}=\mathcal{B}\left(\mathbb{C}^{d}\right) \otimes \mathfrak{M}_{\mathcal{R}}$, where $\mathfrak{M}_{\mathcal{R}}:=\mathcal{V}_{\mathcal{R}}^{\prime \prime}$.

As explained in Section 4.2, (2.11) defines a one-parameter group $\left\{\tau_{t}\right\}_{t \in \mathbb{R}}$ of $*$-automorphisms on $\mathfrak{M}$. The standard Liouvillean of $\left\{\tau_{t}\right\}_{t \in \mathbb{R}}$ reads

$$
L_{\mathfrak{g}}=i \mathfrak{L}_{\mathrm{at}} \otimes \mathbf{1}_{\mathfrak{H}_{\mathcal{R}}}+\mathbf{1}_{\mathfrak{H}_{\mathrm{at}}} \otimes L_{\mathcal{R}}=\left(\stackrel{H_{\mathrm{at}}}{\longrightarrow}-\underset{H_{\mathrm{at}}}{\longleftarrow}\right) \otimes \mathbf{1}_{\mathfrak{H}_{\mathcal{R}}}+\mathbf{1}_{\mathfrak{H}_{\mathrm{at}}} \otimes \mathrm{d} \Gamma(p)
$$

in the representation given above and satisfies (4.5). See also (2.8) and (4.22). For any $A \in \mathfrak{H}_{\mathrm{Hat}} \equiv \mathcal{B}\left(\mathbb{C}^{d}\right)$ and $B \in \operatorname{Dom}\left(\mathrm{e}^{-\beta L_{\mathcal{R}} / 2}\right) \subset \mathfrak{H}_{\mathcal{R}}$,

$$
\Delta^{1 / 2}(A \otimes B)=\left(\rho_{\mathrm{at}}^{1 / 2} A \rho_{\mathrm{at}}^{-1 / 2}\right) \otimes\left(\mathrm{e}^{-\beta L_{\mathcal{R}} / 2} B\right), \quad J(A \otimes B)=A^{*} \otimes\left(J_{\mathcal{R}} B\right)
$$

with $J_{\mathcal{R}}$ being the modular conjugation associated with the pair $\left(\mathfrak{M}_{\mathcal{R}}, \Omega_{\mathcal{R}}\right)$. In fact,

$$
J_{\mathcal{R}} \tilde{\Phi}\left(g_{f}\right) J_{\mathcal{R}}=(-1)^{\mathrm{d} \Gamma\left(\mathbf{1}_{\mathfrak{h}_{2}}\right)} \tilde{\Phi}\left(g_{f}^{\#}\right), \quad f \in \mathfrak{h}_{1},
$$

where

$$
g_{f}^{\#}(p, \vartheta):=i \overline{g_{f}(-p, \vartheta)}, \quad(p, \vartheta) \in \mathbb{R} \times S^{2} \text { (a.e.) } .
$$

Compare with (2.15). For more details, see [15, Theorem 3.3. and Proposition 3.4.].

In the same representation, the time-dependent $C$-Liouvillean $\mathcal{L}_{t}$ (Definition 4.3) then equals

$$
\mathcal{L}_{t} \equiv \mathcal{L}_{t}^{(\lambda, \eta)}:=L_{\mathfrak{g}}+W_{t}-J \Delta^{1 / 2} W_{t} \Delta^{-1 / 2} J
$$


with

$$
\begin{aligned}
W_{t}:=\eta \cos (\varpi t) & \stackrel{H_{\mathrm{p}}}{\longrightarrow} \otimes \mathbf{1}_{\mathcal{V}_{\mathcal{R}}}+\lambda \sum_{\kappa=1}^{\mathrm{K}} \sum_{\ell_{1}, \ldots, \ell_{\kappa}=1}^{m} \stackrel{Q_{\ell_{1}, \ldots, \ell_{\kappa}}^{(\kappa)}}{\longrightarrow} \otimes \\
& \left(\frac{1}{\sqrt{2}}\right)^{\kappa}\left(a^{+}\left(g_{\ell_{1}}^{(\kappa)}\right)+a\left(g_{\ell_{1}}^{(\kappa)}\right)\right) \cdots\left(a^{+}\left(g_{\ell_{\kappa}}^{(\kappa)}\right)+a\left(g_{\ell_{\kappa}}^{(\kappa)}\right)\right)
\end{aligned}
$$

and, by (4.25) (4.26),

$$
\begin{aligned}
J \Delta^{1 / 2} W_{t} \Delta^{-1 / 2} J= & \eta \cos (\varpi t) \rho_{\rho_{\mathrm{at}}^{-1 / 2} H_{\mathrm{p}} \rho_{\mathrm{at}}^{1 / 2}}^{\longleftarrow} \mathbf{1}_{\mathcal{V}_{\mathcal{R}}}-\lambda \sum_{\kappa=1}^{\mathrm{K}} \sum_{\ell_{1}, \ldots, \ell_{\kappa}=1}^{m} \stackrel{\rho_{\mathrm{at}}^{-1 / 2} Q_{\ell_{1}, \ldots, \ell_{\kappa}}^{(\kappa)} \rho_{\mathrm{at}}^{1 / 2}}{{ }^{\prime}} \otimes \\
& \left(\frac{-i}{\sqrt{2}}\right)^{\kappa}(-1)^{\mathrm{d} \Gamma\left(\mathbf{1}_{\mathfrak{h}_{2}}\right)}\left(a\left(i \mathrm{e}^{-\beta p} g_{\ell_{1}}^{(\kappa)}\right)+a^{+}\left(i g_{\ell_{1}}^{(\kappa)}\right)\right) \\
& \cdots(-1)^{\mathrm{d} \Gamma\left(\mathbf{1}_{\mathfrak{h}_{2}}\right)}\left(a\left(i \mathrm{e}^{-\beta p} g_{\ell_{\kappa}}^{(\kappa)}\right)+a^{+}\left(i g_{\ell_{\kappa}}^{(\kappa)}\right)\right) .
\end{aligned}
$$

Recall that $\rho_{\text {at }} \in \mathcal{B}\left(\mathbb{C}^{d}\right)$ is a (invertible) density matrix of the (faithful) state $\omega_{\text {at }}$ with $\left[H_{\text {at }}, \rho_{\text {at }}\right]=0$ and, for any $\kappa=\{1, \ldots, \mathrm{K}\}$ and $\ell \in\{1, \ldots, m\}$ with $\mathrm{K}, m \in \mathbb{N}$,

$$
g_{\ell}^{(\kappa)}(p, \vartheta):=\mathrm{g}_{\ell}^{(\kappa)}(p), \quad(p, \vartheta) \in \mathbb{R} \times S^{2},
$$

where $\left\{\mathrm{g}_{\ell}^{(\kappa)}\right\}_{\ell=1}^{m}$ is the family of complex-valued functions defined on $\mathbb{R}$ by (2.14). Note that $\mathrm{d} \Gamma\left(\mathbf{1}_{\mathfrak{h}_{2}}\right)$, the second quantization of $\mathbf{1}_{\mathfrak{h}_{2}}$, is the particle number operator acting on the antisymmetric Fock space $\mathfrak{H}_{\mathcal{R}}:=\mathcal{F}_{-}\left(\mathfrak{h}_{2}\right)$.

$\left\{W_{t}\right\}_{t \in \mathbb{R}} \in C^{\infty}(\mathbb{R}, \mathcal{V})$ is $2 \pi \varpi^{-1}$-periodic and the operators

$$
V_{t}:=W_{t}-J \Delta^{1 / 2} W_{t} \Delta^{-1 / 2} J
$$

defines a smooth family $\left\{V_{t}\right\}_{t \in \mathbb{R}} \in C^{\infty}(\mathbb{R}, \mathcal{B}(\mathfrak{H}))$. Therefore, the assumptions of Proposition 4.5 are satisfied for this explicit example. The evolution family $\left\{U_{s, t}\right\}_{s, t \in \mathbb{R}}$ is in this case $2 \pi \varpi^{-1}$-periodic, by Proposition 4.4 (v).

\subsection{Evolution group of $\left\{U_{s, t}^{(\lambda, \eta)}\right\}_{t \geq s}$}

We now represent the non-autonomous evolution family $\left\{U_{s, t} \equiv U_{s, t}^{(\lambda, \eta)}\right\}_{s, t \in \mathbb{R}}$ of Proposition 4.4 as an autonomous dynamics on the enlarged Hilbert space

$$
\mathcal{H}:=L^{2}\left(\left[0,2 \pi \varpi^{-1}\right), \mathfrak{H}\right)
$$

of $2 \pi \varpi^{-1}$-periodic $\mathfrak{H}$-valued functions. The same procedure is used in [13, Section 3.4] and thus there is some overlap between the latter and the present section. However, as the time/coupling/frequency regime considered here is completely different to the one studied in 13. (see Introduction), various important technical aspects are different. See, for instance, discussion after Lemma 4.8, In spite of such an overlap with [13, Section 3.4], for the reader's convenience, we prove all statements we use in this section, because their proofs are rather short. The scalar product on $\mathcal{H}$ is naturally defined by

$$
\langle f, g\rangle_{\mathcal{H}}:=\frac{\varpi}{2 \pi} \int_{0}^{\frac{2 \pi}{\varpi}}\langle f(t), g(t)\rangle_{\mathfrak{H}} \mathrm{d} t, \quad f, g \in \mathcal{H}
$$

In the sequel we identify $\mathfrak{H}$ with the subspace of constant functions on $\left[0,2 \pi \varpi^{-1}\right)$. See also (4.23).

From the strongly continuous two-parameter family $\left\{U_{s, t}\right\}_{s, t \in \mathbb{R}}$ on $\mathfrak{H}$ we define a strongly continuous oneparameter group $\left\{\mathfrak{T}_{\alpha}\right\}_{\alpha \in \mathbb{R}}$ on $\mathcal{H}$ by the condition

$$
\forall t \in\left[0,2 \pi \varpi^{-1}\right) \text { (a.e.) : } \quad \mathfrak{T}_{\alpha}(f)(t)=U_{t, t+\alpha} f(t+\alpha),
$$

for all $\alpha \in \mathbb{R}$ and $f \in \mathcal{H}$. Because of (4.28) and Proposition $4.4(\mathrm{v}), \mathfrak{T}_{\alpha}$ is an operator acting on $\mathcal{H}$ for any $\alpha \in \mathbb{R}$. The strong continuity of $\alpha \mapsto \mathfrak{T}_{\alpha}$ follows from the strong continuity of $t \mapsto U_{s, t}$, and the group property of $\left\{\mathfrak{T}_{\alpha}\right\}_{\alpha \in \mathbb{R}}$ from Proposition 4.4 (i). 
The Howland operator of the non-autonomous dynamics $\left\{U_{s, t}\right\}_{s, t \in \mathbb{R}}$ is, by definition, the generator $\mathcal{G}$ of the strongly continuous group $\left\{\mathfrak{T}_{\alpha}\right\}_{\alpha \in \mathbb{R}}$. It is a closed unbounded operator acting on $\mathcal{H}$. It is not a priori clear whether the group $\left\{\mathfrak{T}_{\alpha}\right\}_{\alpha \in \mathbb{R}}$ is contractive. In fact, it is quasi-contractive. Such a property can be useful to analyze the domain of generators of semigroups.

We show that $( \pm \mathcal{G}-C)$ is dissipative, i.e.,

$$
\forall f \in \operatorname{Dom}(\mathcal{G}): \quad \operatorname{Re}\left\{\langle f,( \pm \mathcal{G}-C) f\rangle_{\mathcal{H}}\right\} \leq 0,
$$

for a sufficiently large positive constant $C$. By the Lumer-Phillips theorem, $( \pm \mathcal{G}-C)$ generate contraction semigroups.

\section{Lemma 4.6 (Quasi-Contractivity of $\left\{\mathfrak{T}_{\alpha}\right\}_{\alpha \in \mathbb{R}}$ )}

There is $C \in \mathbb{R}_{0}^{+}$such that $( \pm \mathcal{G}-C)$ is dissipative. In particular, $\left\{\mathrm{e}^{-C \alpha} \mathfrak{T}_{\alpha}\right\}_{\alpha \geq 0}$ and $\left\{\mathrm{e}^{\left.-C \alpha \mathfrak{T}_{-\alpha}\right\}_{\alpha \geq 0}}\right.$ are contraction semigroups.

Proof. For any positive constant $C \in \mathbb{R}_{0}^{+}$, we define the operators

$$
U_{s, t}^{(C)}:=U_{s, t} \mathrm{e}^{(s-t) C}, \quad s, t \in \mathbb{R} .
$$

By Proposition 4.4 and Equations (4.24) and (4.27), $\left\{U_{s, t}^{(C)}\right\}_{s, t \in \mathbb{R}}$ is the fundamental solution on $\operatorname{Dom}\left(L_{\mathfrak{g}}\right)$ of the Cauchy initial value problem

$$
\forall s, t \in \mathbb{R}: \quad \partial_{s} U_{s, t}^{(C)}=-\left(i \mathcal{L}_{s}-C\right) U_{s, t}^{(C)}, \quad U_{t, t}^{(C)}=\mathbf{1}_{\mathfrak{H}},
$$

while it solves on $\operatorname{Dom}\left(L_{\mathfrak{g}}\right)$ the Cauchy initial value problem

$$
\forall s, t \in \mathbb{R}: \quad \partial_{t} U_{s, t}^{(C)}=U_{s, t}^{(C)}\left(i \mathcal{L}_{t}-C\right), \quad U_{s, s}^{(C)}=\mathbf{1}_{\mathfrak{H}} .
$$

Choose

$$
C:=\max _{t \in \mathbb{R}}\left\|V_{t}\right\|_{\mathcal{B}(\mathfrak{H})}<\infty
$$

where we recall that $V_{t}$ is defined by (4.31). As $L_{\mathfrak{g}}$ is self-adjoint, $\left(i \mathcal{L}_{t}-C\right)$ is dissipative for all $t \in \mathbb{R}$ and 23. Theorem 4.8.] implies that $\left\|U_{s, t}^{(C)}\right\|_{\mathcal{B}(\mathfrak{H})} \leq 1$ for all $t \geq s$. It follows that $\left\|\mathrm{e}^{-C \alpha \mathfrak{T}_{\alpha}}\right\|_{\mathcal{B}(\mathcal{H})} \leq 1$ for all $\alpha \geq 0$. Similarly, we show that $\left\|\mathrm{e}^{-C \alpha} \mathfrak{T}_{-\alpha}\right\|_{\mathcal{B}(\mathcal{H})} \leq 1$ for all $\alpha \geq 0$. To finish the proof, one uses the fact that $( \pm \mathcal{G}-C)$ is the generator of the contraction semigroup $\left\{\mathrm{e}^{-C \alpha} \mathfrak{T}_{ \pm \alpha}\right\}_{\alpha \geq 0}$ together with the Lumer-Phillips theorem.

Observe that $\mathcal{H}$ is unitarily equivalent - via the Fourier transform $\mathfrak{F}-$ to the Hilbert space $\hat{\mathcal{H}}:=\ell^{2}(\mathbb{Z}, \mathfrak{H})$ with scalar product

$$
\langle\hat{f}, \hat{g}\rangle_{\hat{\mathcal{H}}}:=\sum_{k \in \mathbb{Z}}\langle\hat{f}(k), \hat{g}(k)\rangle_{\mathfrak{H}}, \quad \hat{f}, \hat{g} \in \hat{\mathcal{H}}
$$

It is indeed more convenient to analyze the Fourier transform $\mathfrak{F G F} \mathfrak{F}^{*}$ instead of $\mathcal{G}$ directly. To this end, we define the dense subspace

$$
\hat{\mathcal{D}}:=\left\{\hat{f} \in \hat{\mathcal{H}}: \hat{f}(\mathbb{Z}) \subset \operatorname{Dom}\left(L_{\mathfrak{g}}\right), \sum_{k \in \mathbb{Z}}\left(k^{2}\|\hat{f}(k)\|_{\mathfrak{H}}^{2}+\left\|L_{\mathfrak{g}}(\hat{f}(k))\right\|_{\mathfrak{H}}^{2}\right)<\infty\right\}
$$

of the Hilbert space $\hat{\mathcal{H}}$ as well as the (unbounded) operators $k_{\hat{\mathcal{H}}}$ and $L_{\mathfrak{g}, \hat{\mathcal{H}}}$ on $\hat{\mathcal{D}}$ by

$$
\forall k \in \mathbb{Z}: \quad\left(k_{\hat{\mathcal{H}}} \hat{f}\right)(k):=k \hat{f}(k), \quad\left(L_{\mathfrak{g}, \hat{\mathcal{H}}} \hat{f}\right)(k):=L_{\mathfrak{g}} \hat{f}(k),
$$

see (4.24). By abuse of notation, we denote $k_{\hat{\mathcal{H}}}$ and $L_{\mathfrak{g}, \hat{\mathcal{H}}}$ respectively by $k$ and $L_{\mathfrak{g}}$. In the same way, let $V_{\mathcal{H}} \equiv V$ be the bounded operator acting on $\mathcal{H}$ defined by

$$
\forall t \in\left[0,2 \pi \varpi^{-1}\right)(\text { a.e. }): \quad\left(V_{\mathcal{H}} f\right)(t):=V_{t}(f(t)) .
$$

We prove below that the unbounded operator

$$
\hat{\mathcal{G}}:=i\left(\varpi k+L_{\mathfrak{g}}+\hat{V}\right)
$$

defined on $\hat{\mathcal{D}}$ with $\hat{V}:=\mathfrak{F} V \mathfrak{F}^{*}$ is the Fourier transform $\mathfrak{F G} \mathfrak{F}^{*}$ of $\mathcal{G}$ : 
Theorem 4.7 (Explicit form of the Howland operator in Fourier space)

The Fourier transform of the generator $\mathcal{G}$ of the strongly continuous group $\left\{\mathfrak{T}_{\alpha}\right\}_{\alpha \in \mathbb{R}}$ equals

$$
\mathfrak{F G \mathfrak { F } ^ { * }}=\hat{\mathcal{G}} .
$$

Proof. The operator $\varpi k+L_{\mathfrak{g}}$ can be viewed as a tensor sum of self-adjoint operators acting on

$$
\hat{\mathcal{H}}=\bigoplus_{k \in \mathbb{Z}} \mathfrak{H}_{k}, \quad \mathfrak{H}_{k} \equiv \mathfrak{H}
$$

It is essentially self-adjoint on

$$
\hat{\mathcal{D}}_{0}:=\left\{\hat{f} \in \hat{\mathcal{H}}: \hat{f}(k)=0 \text { for } k \text { outside a finite set and } \hat{f}(\mathbb{Z}) \subset \operatorname{Dom}\left(L_{\mathfrak{g}}\right)\right\} \subset \hat{\mathcal{D}}
$$

and $\hat{\mathcal{D}}$ is the graph norm closure of $\hat{\mathcal{D}}_{0}$ w.r.t. the operator $\varpi k+L_{\mathfrak{g}}$. Hence, $\varpi k+L_{\mathfrak{g}}$ is self-adjoint on $\hat{\mathcal{D}}$. Since $\left\{V_{t}\right\}_{t \in \mathbb{R}} \in C^{\infty}(\mathbb{R}, \mathcal{B}(\mathfrak{H}))$, the operator $\hat{V} \in \mathcal{B}(\hat{\mathcal{H}})$ is bounded. Thus, $\hat{\mathcal{G}}$ is closed on $\hat{\mathcal{D}}$. The unbounded part $i\left(\varpi k+L_{\mathfrak{g}}\right)$ of $\hat{\mathcal{G}}$ is dissipative, as $\varpi k+L_{\mathfrak{g}}$ is self-adjoint. Hence, by adding a sufficiently large constant $C \geq\|\hat{V}\|_{\mathcal{B}(\hat{\mathcal{H}})}$, the operator $(\hat{\mathcal{G}}-C)$ defined on the dense set $\hat{\mathcal{D}}$ is the dissipative generator of a strongly continuous semigroup. On the other hand, as $\mathfrak{F} \mathcal{G} \mathfrak{F}^{*}=\hat{\mathcal{G}}$ on the core $\hat{\mathcal{D}}_{0}$ of $(\hat{\mathcal{G}}-C),\left(\mathfrak{F} \mathcal{G} \mathfrak{F}^{*}-C\right)$ is a closed extension of $(\hat{\mathcal{G}}-C)$ and generates a strongly continuous semigroup. Choosing $C$ sufficiently large, both generators $(\hat{\mathcal{G}}-C)$ and $\left(\mathfrak{F G} \mathfrak{F}^{*}-C\right)$ are dissipative, by Lemma 4.6. Using the fact that generators of contraction semigroups have no proper dissipative extensions, it follows that $\hat{\mathcal{G}}=\mathfrak{F} \mathcal{G} \mathfrak{F}^{*}$.

We show next that - at small couplings - the quantity

$$
\left\langle\Omega, U_{0, t}\left(A \otimes \mathbf{1}_{\mathfrak{H}_{\mathcal{R}}} \Omega\right)\right\rangle_{\mathfrak{H}},
$$

which gives the time evolution of the atomic state, are well-approximated by

$$
\left\langle\Omega, \mathfrak{T}_{t}\left(A \otimes \mathbf{1}_{\mathfrak{H}_{\mathcal{R}}} \Omega\right)\right\rangle_{\mathcal{H}},
$$

provided the density matrix $\rho_{\text {at }}$ of the initial atomic state as well as the atomic observable $A$ are block diagonal (cf. (3.4)):

Theorem 4.8 (Effective behavior of $\rho(t)$ )

For any initial faithful state $\omega_{\text {at }}$ with density matrix $\rho_{\text {at }} \in \mathfrak{D} \subset \mathcal{B}\left(\mathbb{C}^{d}\right)$, any observable $A \in \mathfrak{D}$, and $\alpha \in \mathbb{R}$,

$$
\left|\left\langle\Omega, U_{0, \alpha}\left(A \otimes \mathbf{1}_{\mathfrak{H}_{\mathcal{R}}} \Omega\right)\right\rangle_{\mathfrak{H}}-\left\langle\Omega, \mathfrak{T}_{\alpha}\left(A \otimes \mathbf{1}_{\mathfrak{H}_{\mathcal{R}}} \Omega\right)\right\rangle_{\mathcal{H}}\right| \leq C\|A\|_{\mathcal{B}\left(\mathbb{C}^{d}\right)}|\lambda|(1+|\lambda|) \varpi^{-1} \mathrm{e}^{D(1+|\lambda|)^{2}},
$$

where $C, D \in \mathbb{R}_{0}^{+}$are finite constants not depending on $\omega_{\mathrm{at}}, A, \lambda, \eta, \varpi$, and $\alpha$.

Proof. Since

$$
\left\langle\Omega, U_{0, \alpha}\left(A \otimes \mathbf{1}_{\mathfrak{H}_{\mathcal{R}}} \Omega\right)\right\rangle_{\mathfrak{H}}-\left\langle\Omega, \mathfrak{T}_{\alpha}\left(A \otimes \mathbf{1}_{\mathfrak{H}_{\mathcal{R}}} \Omega\right)\right\rangle_{\mathcal{H}}=\frac{\varpi}{2 \pi} \int_{0}^{\frac{2 \pi}{\varpi}}\left\langle\Omega,\left(U_{0, \alpha}-U_{t, t+\alpha}\right)\left(A \otimes \mathbf{1}_{\mathfrak{H}_{\mathcal{R}}} \Omega\right)\right\rangle_{\mathfrak{H}} \mathrm{d} t
$$

we need to estimate the difference

$$
\left\langle\Omega,\left(U_{0, \alpha}-U_{t, t+\alpha}\right)\left(A \otimes \mathbf{1}_{\mathfrak{H}_{\mathcal{R}}} \Omega\right)\right\rangle_{\mathfrak{H}}
$$

for any $\alpha \in \mathbb{R}$ and $t \in\left[0,2 \pi \varpi^{-1}\right)$. Using Proposition 4.4 (i), note that

$$
U_{0, \alpha}-U_{t, t+\alpha}=U_{0, \alpha}\left(\mathbf{1}_{\mathfrak{H}}-U_{\alpha, t+\alpha}\right)+\left(U_{0, t}-\mathbf{1}_{\mathfrak{H}}\right) U_{t, t+\alpha} .
$$

By Proposition 4.5

$$
\begin{aligned}
\left\langle\Omega,\left(U_{0, \alpha}-U_{t, t+\alpha}\right)\left(A \otimes \mathbf{1}_{\mathfrak{H}_{\mathcal{R}}} \Omega\right)\right\rangle_{\mathfrak{H}}= & \left\langle\Omega, \mathfrak{W}_{0, \alpha}\left(A \otimes \mathbf{1}_{\mathfrak{H}_{\mathcal{R}}}-\mathfrak{W}_{\alpha, t+\alpha}\left(A \otimes \mathbf{1}_{\mathfrak{H}_{\mathcal{R}}}\right)\right) \Omega\right\rangle_{\mathfrak{H}} \\
& +\left\langle\Omega,\left(U_{0, t}-\mathbf{1}_{\mathfrak{H}}\right) U_{t, t+\alpha}\left(A \otimes \mathbf{1}_{\mathfrak{H}_{\mathcal{R}}} \Omega\right)\right\rangle_{\mathfrak{H}}
\end{aligned}
$$


for any $t \in\left[0,2 \pi \varpi^{-1}\right)$. On the one hand, for any $B \in \mathcal{V}$,

$$
\left|\left\langle\Omega, \mathfrak{W}_{0, \alpha}\left(B-\mathfrak{W}_{\alpha, t+\alpha}(B)\right) \Omega\right\rangle_{\mathfrak{H}}\right| \leq\left\|\mathfrak{W}_{0, \alpha}\right\|_{\mathcal{B}(\mathcal{V})}\left\|B-\mathfrak{W}_{\alpha, t+\alpha}(B)\right\|_{\mathcal{V}}=\left\|B-\mathfrak{W}_{\alpha, t+\alpha}(B)\right\|_{\mathcal{V}} .
$$

By (4.3)-4.4),

$$
\begin{aligned}
\left\|B-\mathfrak{W}_{\alpha, t+\alpha}(B)\right\|_{\mathcal{V}} \leq & \left\|\mathfrak{V}_{t+\alpha, \alpha}-\mathbf{1}_{\mathcal{V}}\right\|_{\mathcal{V}}\|B\|_{\mathcal{V}}\left\|\mathfrak{V}_{t+\alpha, \alpha}^{*}\right\|_{\mathcal{V}}+\|B\|_{\mathcal{V}}\left\|\mathfrak{V}_{t+\alpha, \alpha}^{*}-\mathbf{1}_{\mathcal{V}}\right\|_{\mathcal{V}} \\
& +\left\|\tau_{t}(B)-B\right\|_{\mathcal{V}}\left\|\mathfrak{V}_{t+\alpha, \alpha}^{*}\right\|_{\mathcal{V}}
\end{aligned}
$$

with

$$
\mathfrak{V}_{t+\alpha, \alpha}=\mathbf{1}_{\mathcal{V}}+\sum_{k \in \mathbb{N}} i^{k} \int_{0}^{t} \mathrm{~d} s_{1} \cdots \int_{0}^{s_{k-1}} \mathrm{~d} s_{k} \tau_{s_{k}+\alpha}\left(\tau_{\alpha}\left(W_{s_{k}+\alpha}\right)\right) \cdots \tau_{s_{1}}\left(\tau_{\alpha}\left(W_{s_{1}+\alpha}\right)\right) .
$$

Since $\tau_{t}\left(A \otimes \mathbf{1}_{\mathfrak{H}_{\mathcal{R}}}\right)=A \otimes \mathbf{1}_{\mathfrak{H}_{\mathcal{R}}}$ for $A \in \mathfrak{D} \subset \mathcal{B}\left(\mathbb{C}^{d}\right)$, it follows from (4.40)-(4.42) together with (4.28) and Assumption 3 that

$$
\left|\left\langle\Omega, \mathfrak{W}_{0, \alpha}\left(A \otimes \mathbf{1}_{\mathfrak{H}_{\mathcal{R}}}-\mathfrak{W}_{\alpha, t+\alpha}\left(A \otimes \mathbf{1}_{\mathfrak{H}_{\mathcal{R}}}\right)\right) \Omega\right\rangle_{\mathfrak{H}}\right| \leq \frac{|\lambda|(1+|\lambda|)}{\varpi} C\|A\|_{\mathcal{V}} .
$$

On the other hand, for any $B \in \mathcal{V}$,

$$
\left|\left\langle\Omega,\left(U_{0, t}-\mathbf{1}_{\mathfrak{H}}\right) U_{t, t+\alpha}(B \Omega)\right\rangle_{\mathfrak{H}}\right| \leq\left\|\left(U_{0, t}^{*}-\mathbf{1}_{\mathfrak{H}}\right) \Omega\right\|_{\mathfrak{H}}\|B\|_{\mathcal{V}} .
$$

Meanwhile, we deduce from (4.15) that

$$
\forall s, t \in \mathbb{R}: \quad U_{s, t}^{*}=\mathrm{e}^{i(s-t) L_{\mathfrak{g}}}-i\left(\int_{s}^{t} U_{s, r} V_{r} \mathrm{e}^{i(r-t) L_{\mathfrak{g}}} \mathrm{d} r\right)^{*}
$$

while

$$
\forall s, t \in \mathbb{R}: \quad \mathrm{e}^{i(s-t) L_{\mathfrak{g}}} \Omega=\Omega+i \int_{t}^{s} \mathrm{e}^{-i r L_{\mathfrak{g}}} L_{\mathfrak{g}} \Omega \mathrm{d} r,
$$

because $\Omega \in \operatorname{Dom}\left(L_{\mathfrak{g}}\right)$. If $\rho_{\text {at }} \in \mathfrak{D}$ then $L_{\mathfrak{g}} \Omega=0$. So, we can combine (4.44) with (4.45)-(4.46) and the upper bound [23, Theorem 4.8.]

$$
\left\|U_{t, s}\right\|_{\mathcal{B}(\mathfrak{H})} \leq C \mathrm{e}^{D(t-s)}
$$

to arrive at

$$
\left|\left\langle\Omega,\left(U_{0, t}-\mathbf{1}_{\mathfrak{H}}\right) U_{t, t+\alpha}\left(A \otimes \mathbf{1}_{\mathfrak{H}_{\mathcal{R}}} \Omega\right)\right\rangle_{\mathfrak{H}}\right| \leq C\|A\|_{\mathcal{V}}|\lambda|(1+|\lambda|) \varpi^{-1} \mathrm{e}^{D(1+|\lambda|)^{2}} .
$$

Finally, using (4.38), (4.39), (4.43), and (4.47) we obtain the assertion.

The above estimate is similar to [13, Lemma 3.3]. But, in contrast to the latter, the above lemma gives a bound which is uniform in time. Recall that, as explained in Section 1, [13] and the present work consider completely different regimes of couplings and times. Note also that we use, in an essential way, the equality $\left[\rho_{\text {at }}, H_{\text {at }}\right]=0$ in the proof of Theorem 4.8 .

\subsection{Resonances of the Howland operator}

Similar to [15], we now perform an analytic deformation of the Howland operator in Fourier space (cf. see (4.35) and Theorem 4.7) and study its spectrum after deformation. Note again that the same technique was also used in [13] and there are some common issues between the present Section and [13, Section 3.5]. Nevertheless, as already stressed many times, the considered regimes are very different and the studies of spectral properties of the deformed Howland operator are, from a technical point of view, not the same.

Let

$$
\hat{\mathcal{G}}_{0}(\theta):=i\left(\varpi k+L_{\mathfrak{g}}+\theta \hat{N}\right), \quad \theta \in \mathbb{C},
$$

where, for any $\hat{f} \in \hat{\mathcal{H}}$ and $k \in \mathbb{Z}$,

$$
\hat{N}(\hat{f})(k):=\mathbf{1}_{\mathfrak{H}_{\text {at }}} \otimes \mathrm{d} \Gamma\left(\mathbf{1}_{\mathfrak{h}_{2}}\right)(\hat{f}(k)) .
$$


Recall that $\mathrm{d} \Gamma\left(\mathbf{1}_{\mathfrak{h}_{2}}\right)$ is the second quantization of $\mathbf{1}_{\mathfrak{h}_{2}}$, i.e., the particle number operator acting on $\mathfrak{H}_{\mathcal{R}}:=\mathcal{F}_{-}\left(\mathfrak{h}_{2}\right)$. For all $\theta \in \mathbb{C}$ such that $\operatorname{Im}\{\theta\}>0, \hat{\mathcal{G}}_{0}$ is a normal operator with domain

$$
\hat{\mathcal{D}} \cap \operatorname{Dom}(\hat{N})=\operatorname{Dom}\left(\hat{\mathcal{G}}_{0}(\theta)\right)
$$

and spectrum in the left half-plane. In particular, by the spectral theorem for normal operators, $\hat{\mathcal{G}}_{0}(\theta)$ is the generator of a strongly continuous contraction semigroup for all $\theta \in \mathbb{C}$ such that $\operatorname{Im}\{\theta\} \geq 0$. [It cannot be extended to a group, as the (negative) real part of the spectrum of $\hat{\mathcal{G}}_{0}(\theta)$ is unbounded.]

Similarly, we define the operator $\hat{V}(\theta)$ by replacing in Equations (4.28)-(4.31) the functions $g_{\ell}^{(\kappa)}$ with

$$
\tilde{g}_{\ell, \theta}^{(\kappa)}(p, \vartheta):=g_{\ell}^{(\kappa)}(p+\theta, \vartheta), \quad(p, \vartheta) \in \mathbb{R} \times S^{2},
$$

in the creation operators and with $\tilde{g}_{\ell, \bar{\theta}}^{(\kappa)}$ in the annihilation operators for every $\kappa \in\{1, \ldots, \mathrm{K}\}$ and $\ell \in\{1, \ldots, m\}$, see (4.30). Indeed, for real parameters $\theta \in \mathbb{R} \subset \mathbb{C}$, it is easy to see that

$$
\hat{V}(\theta)=U(\theta) \hat{V} U(\theta)^{*},
$$

where $U(\theta), \theta \in \mathbb{R}$, is the unitary operator defined on $\hat{\mathcal{H}}$ by

$$
(U(\theta) \hat{f})(k):=\left(\mathbf{1}_{\mathfrak{H}_{\mathrm{at}}} \otimes \Gamma(u(\theta))(\hat{f}(k))\right.
$$

for any $\hat{f} \in \hat{\mathcal{H}}$ and $k \in \mathbb{Z}$. Here, $\Gamma(u(\theta))$ is the second quantization of the unitary (translation) operator $u(\theta)$ from $\mathfrak{h}_{2}$ to $\mathfrak{h}_{2}$ defined by

$$
(u(\theta) f)(p, \vartheta):=f(p+\theta, \vartheta)
$$

for any $f \in \mathfrak{h}_{2}$ and $(p, \vartheta) \in \mathbb{R} \times S^{2}$ (a.e.). By Assumption 1, there is $\mathrm{r}_{\max } \in \mathbb{R}^{+}$such that, for all $\theta \in \mathbb{C}$ such that $|\operatorname{Im}\{\theta\}|<\mathrm{r}_{\max }, \hat{V}(\theta)$ is a well-defined bounded operator. The deformed Howland operator

$$
\hat{\mathcal{G}}(\theta):=\hat{\mathcal{G}}_{0}(\theta)+i \hat{V}(\theta), \quad \theta \in \mathbb{S}, \quad \mathbb{S}:=\mathbb{R}+i\left[0, \mathrm{r}_{\max }\right)
$$

is thus the generator of a strongly continuous semigroup $\left\{\mathrm{e}^{\hat{\mathcal{G}}(\theta) \alpha}\right\}_{\alpha \geq 0}$. Let $C_{\theta} \in \mathbb{R}_{0}^{+}$and $D_{\theta} \in[1, \infty)$ be the stability constants of $\hat{\mathcal{G}}(\theta)$, i.e.,

$$
\left\|\mathrm{e}^{\hat{\mathcal{G}}(\theta) \alpha}\right\|_{\hat{\mathcal{H}}} \leq D_{\theta} \mathrm{e}^{\alpha C_{\theta}}
$$

The family $\{\hat{\mathcal{G}}(\theta)\}_{\theta \in \mathbb{S} \backslash \mathbb{R}}$ of closed operators is of type A (Definition 5.1). See also 24]. This property is an obvious consequence of the following lemma:

Lemma 4.9 (Analyticity of $\hat{V}(\cdot)$ )

The map $\theta \mapsto \hat{V}(\theta)$ from $\mathbb{R}+i\left(-\mathrm{r}_{\max }, \mathrm{r}_{\max }\right) \subset \mathbb{C}$ to $\mathcal{B}(\hat{\mathcal{H}})$ is analytic.

Proof. Define, for all $\kappa=\{1, \ldots, \mathrm{K}\}$ and $\ell \in\{1, \ldots, m\}$, the maps

$$
\psi_{\ell}^{(\kappa)}, \psi_{\ell,+}^{(\kappa)}, \psi_{\ell,-}^{(\kappa)}: \mathbb{R}+i\left(-\mathrm{r}_{\max }, \mathrm{r}_{\max }\right) \rightarrow \mathfrak{h}_{2}:=L^{2}\left(\mathbb{R} \times S^{2}, \mathbb{C}\right)
$$

by

$$
\begin{aligned}
& {\left[\psi_{\ell}^{(\kappa)}(\theta)\right](p, \vartheta):=\mathrm{g}_{\ell}^{(\kappa)}(p+\theta),} \\
& {\left[\psi_{\ell,+}^{(\kappa)}(\theta)\right](p, \vartheta):=\mathrm{e}^{\frac{\beta}{2}(p+\theta)} \mathrm{g}_{\ell}^{(\kappa) \#}(p+\theta, \vartheta),} \\
& {\left[\psi_{\ell,-}^{(\kappa)}(\theta)\right](p, \vartheta):=\mathrm{e}^{-\frac{\beta}{2}(p+\theta)} \mathrm{g}_{\ell}^{(\kappa) \#}(p+\theta, \vartheta)}
\end{aligned}
$$

These three maps are weakly continuous. Indeed, by analyticity of $\mathrm{g}_{\ell}^{(\kappa)}$, for any fixed $\psi \in \mathfrak{h}_{2}, \theta, \theta^{\prime} \in$ $\mathbb{R}+i\left(-\mathrm{r}_{\max }, \mathrm{r}_{\max }\right)$ and some sufficiently small radius $\mathfrak{r}>0$ with $\left|\theta^{\prime}-\theta\right|<\mathfrak{r}$,

$$
\left\langle\psi, \psi_{\ell}^{(\kappa)}\left(\theta^{\prime}\right)\right\rangle_{\mathfrak{h}_{2}}=\frac{1}{2 \pi} \int_{\mathbb{R}} \mathrm{d} p \int_{S^{2}} \mathrm{~d} \vartheta \int_{-\pi}^{\pi} \mathrm{d} \varphi \psi(p, \vartheta) \mathrm{g}_{\ell}^{(\kappa)}\left(p+\theta+\mathrm{re}^{i \varphi}, \vartheta\right) \frac{\mathfrak{r e}^{i \varphi}}{\mathfrak{r e}^{i \varphi}+\theta-\theta^{\prime}} .
$$


By Assumption 1 and the Cauchy-Schwarz inequality,

$$
\int_{-\pi}^{\pi} \mathrm{d} \varphi \int_{S^{2}} \mathrm{~d} \vartheta \int_{\mathbb{R}} \mathrm{d} p\left|\psi(p, \vartheta) \mathrm{g}_{\ell}^{(\kappa)}\left(p+\theta+\mathfrak{r e}^{i \varphi}, \vartheta\right)\right|<\infty
$$

Thus, by the Fubini theorem and the Lebesgue dominated convergence theorem, the map $\theta \mapsto\left\langle\psi, \psi_{\ell}^{(\kappa)}(\theta)\right\rangle_{\mathfrak{h}_{2}}$ is continuous on $\mathbb{R}+i\left(-\mathrm{r}_{\max }, \mathrm{r}_{\max }\right)$. The weak continuity of $\psi_{\ell,+}^{(\kappa)}$ and $\psi_{\ell,-}^{(\kappa)}$ is shown in the same way. Let $\gamma$ be any closed contour in the domain $\mathbb{R}+i\left(-\mathrm{r}_{\max }, \mathrm{r}_{\max }\right) \subset \mathbb{C}$ (with finite length). For any fixed $\psi \in \mathfrak{h}_{2}$, we infer from a similar estimate as (4.53), the Fubini theorem and the analyticity of $\mathrm{g}_{\ell}^{(\kappa)}(\cdot, \vartheta)$ that

$$
\begin{aligned}
\int_{\gamma} \mathrm{d} z\left\langle\psi, \psi_{\ell}^{(\kappa)}(z)\right\rangle_{\mathfrak{h}_{2}} & =\int_{\gamma} \mathrm{d} z \int_{\mathbb{R}} \mathrm{d} p \int_{S^{2}} \mathrm{~d} \vartheta \psi(p, \vartheta) \mathrm{g}_{\ell}^{(\kappa)}(p+z, \vartheta) \\
& =\int_{\mathbb{R}} \mathrm{d} p \int_{S^{2}} \mathrm{~d} \vartheta \psi(p, \vartheta) \int_{\gamma} \mathrm{d} z \mathrm{~g}_{\ell}^{(\kappa)}(p+z, \vartheta)=0 .
\end{aligned}
$$

Thus, by Morera's lemma, the map $\theta \mapsto\left\langle\psi, \psi_{\ell}^{(\kappa)}(\theta)\right\rangle_{\mathfrak{h}_{2}}$ is analytic on $\mathbb{R}+i\left(-\mathrm{r}_{\max }, \mathrm{r}_{\max }\right)$. In other words, $\psi_{\ell}^{(\kappa)}$ is weakly analytic and hence, by [12, Chap. III, Theorem 1.37], it is strongly analytic. The (strong) analyticity of $\psi_{\ell,+}^{(\kappa)}, \psi_{\ell,-}^{(\kappa)}$ is shown by the same arguments.

Finally, observe that

$$
i \mathrm{e}^{-\beta p} \mathrm{~g}_{\ell}^{(\kappa)}(p)=\mathrm{e}^{-\frac{\beta}{2} p} \mathrm{~g}_{\ell}^{(\kappa) \#}(p), \quad i \mathrm{~g}_{\ell}^{(\kappa)}(p)=\mathrm{e}^{\frac{\beta}{2} p} \mathrm{~g}_{\ell}^{(\kappa) \#}(p) .
$$

From the analyticity of $\psi_{\ell}^{(\kappa)}, \psi_{\ell,+}^{(\kappa)}, \psi_{\ell,-}^{(\kappa)}$ together with the bounds

$$
\|a(f)\|_{\mathcal{H}},\left\|a^{+}(f)\right\|_{\mathcal{H}} \leq\|f\|_{\mathfrak{h}_{2}}, \quad f \in \mathfrak{h}_{2},
$$

the linearity of $f \mapsto a^{+}(f)$, the antilinearity of $f \mapsto a(f)$, and Equations (4.28) and (4.29), it then follows that the map $\theta \mapsto \hat{V}(\theta)$ is analytic on the domain $\mathbb{R}+i\left(-\mathrm{r}_{\max }, \mathrm{r}_{\max }\right)$, in the sense of $\mathcal{B}(\hat{\mathcal{H}})$.

The subspace $\hat{\mathcal{D}}_{0} \subset \operatorname{Dom}\left(\hat{\mathcal{G}}_{0}(\theta)\right)$ defined by (4.36) is a core of $\hat{\mathcal{G}}_{0}(\theta)$ for all $\theta \in \mathbb{C}$. Hence, for all $\theta \in \mathbb{S}$, by the boundedness of $\hat{V}(\theta), \hat{\mathcal{D}}_{0}$ is also core of $\hat{\mathcal{G}}(\theta)$. This fact implies the following:

\section{Lemma 4.10 (Limit of semigroups)}

For all $\hat{f} \in \hat{\mathcal{H}}, \alpha \in \mathbb{R}_{0}^{+}$, and $\theta \in \mathbb{S}$,

$$
\mathrm{e}^{\hat{\mathcal{G}}(\theta) \alpha} \hat{f}=\lim _{\theta^{\prime} \rightarrow \theta}\left\{\mathrm{e}^{\hat{\mathcal{G}}\left(\theta^{\prime}\right) \alpha} \hat{f}\right\} .
$$

In particular, for all $\hat{f} \in \hat{\mathcal{H}}$ and $\zeta \in \mathbb{C}$ with $\operatorname{Re}\{\zeta\}>C_{\theta}$,

$$
(\zeta-\hat{\mathcal{G}}(\theta))^{-1} \hat{f}=\lim _{\theta^{\prime} \rightarrow \theta}\left\{(\zeta-\hat{\mathcal{G}}(\theta))^{-1} \hat{f}\right\} .
$$

Proof. The first assertion follows from the Trotter-Kato approximation theorem [25, Chap. III, Theorem 4.8] as $\hat{\mathcal{G}}\left(\theta^{\prime}\right)$ converges strongly on the common core $\hat{\mathcal{D}}_{0}$ when $\theta^{\prime} \rightarrow \theta$, by Lemma 4.9. The second assertion results from the integral representation of the resolvent [25, Chap. III, Eq. (5.18)]:

$$
(\zeta-\hat{\mathcal{G}}(\theta))^{-1} \hat{f}=\int_{0}^{\infty} \mathrm{e}^{\hat{\mathcal{G}}(\theta) \alpha} \mathrm{e}^{-\zeta \alpha} \hat{f} \mathrm{~d} \alpha
$$

for all $\zeta \in \mathbb{C}$ with $\operatorname{Re}\{\zeta\}>C_{\theta}$.

Recall that $\mathfrak{H}_{\text {at }}$ is defined by (2.4), while $\Omega_{\mathcal{R}}$ is the vacuum of $\mathfrak{H}_{\mathcal{R}}:=\mathcal{F}_{-}\left(\mathfrak{h}_{2}\right)$ and $\mathfrak{F}$ denotes the Fourier transform. Using Lemma 4.10, we prove now that the dynamics given by $\left\{\mathrm{e}^{\hat{\mathcal{G}}(\theta) \alpha}\right\}_{\alpha \geq 0}$ restricted to the atomic space

$$
\hat{\mathfrak{H}}_{\text {at }}:=\mathfrak{F}\left(\mathfrak{H}_{\text {at }} \otimes \Omega_{\mathcal{R}}\right) \subset \hat{\mathcal{H}}
$$

does not depend on the choice of $\theta \in \mathbb{S}$, in the following sense: 
Theorem 4.11 (Invariance of the evolution semigroup under analytic translations)

For all $\alpha \in \mathbb{R}_{0}^{+}, \hat{\psi}_{1}, \hat{\psi}_{2} \in \hat{\mathfrak{H}}_{\text {at }}$ and $\theta, \theta^{\prime} \in \mathbb{S}$,

$$
\left\langle\hat{\psi}_{1}, \mathrm{e}^{\hat{\mathcal{G}}(\theta) \alpha} \hat{\psi}_{2}\right\rangle_{\hat{\mathcal{H}}}=\left\langle\hat{\psi}_{1}, \mathrm{e}^{\hat{\mathcal{G}}\left(\theta^{\prime}\right) \alpha} \hat{\psi}_{2}\right\rangle_{\hat{\mathcal{H}}} .
$$

Proof. Using (4.54) applied to any vector $\hat{f} \in \hat{\mathfrak{H}}_{\text {at }}$ and the injectivity of the Laplace transform, we only need to show that

$$
\left\langle\hat{\psi}_{1},(\zeta-\hat{\mathcal{G}}(\theta))^{-1} \hat{\psi}_{2}\right\rangle_{\hat{\mathcal{H}}}=\left\langle\hat{\psi}_{1},\left(\zeta-\hat{\mathcal{G}}\left(\theta^{\prime}\right)\right)^{-1} \hat{\psi}_{2}\right\rangle_{\hat{\mathcal{H}}}
$$

for any $\hat{\psi}_{1}, \hat{\psi}_{2} \in \hat{\mathfrak{H}}_{\text {at }}, \theta, \theta^{\prime} \in \mathbb{S}$, and $\zeta \in(D, \infty)$ with

$$
D>\sup _{\theta \in \mathbb{S}} C_{\theta}
$$

see (4.52). Indeed,

$$
\sup _{\theta \in \mathbb{S}} C_{\theta}<\infty
$$

For any real parameter $\theta \in \mathbb{R}$, let the unitary operator $U(\theta)$ be defined by (4.50). Clearly $U(\theta)=U(-\theta)^{*}$ for all $\theta \in \mathbb{R}$ and

$$
\forall \theta \in \mathbb{R}: \quad(\zeta-\hat{\mathcal{G}}(\theta))^{-1}=U(\theta)(\zeta-\hat{\mathcal{G}}(0))^{-1} U(\theta)^{*},
$$

while

$$
\forall \theta \in \mathbb{R}, \hat{\psi} \in \hat{\mathfrak{H}}_{\text {at }}: \quad U(\theta)(\hat{\psi})=\hat{\psi}
$$

It follows that the function

$$
g(\theta):=\left\langle\hat{\psi}_{1},(\zeta-\hat{\mathcal{G}}(\theta))^{-1} \hat{\psi}_{2}\right\rangle_{\hat{\mathcal{H}}}
$$

is constant on $\mathbb{R}$, i.e.,

$$
\forall \theta \in \mathbb{R}: \quad g(\theta)=g(0) .
$$

By Lemma 4.9, the family $\{\hat{\mathcal{G}}(\theta)\}_{\theta \in \mathbb{S} \backslash \mathbb{R}}$ of closed operators is of type A, see Definition [5.1. Therefore, we infer from Lemma 5.2 that the function $g$ is analytic on $\mathbb{S} \backslash \mathbb{R}$. Finally, using the Schwarz reflection principle, we deduce that $g$ is constant on $\mathbb{S}$.

Therefore, as soon as the restricted dynamics on the atom is concerned, we can analyze the evolution given by the strongly continuous semigroup $\left\{\mathrm{e}^{\hat{\mathcal{G}}(i r) \alpha}\right\}_{\alpha \geq 0}$ at a fixed $r \in\left(0, \mathrm{r}_{\max }\right)$. The main advantage of studying $\left\{\mathrm{e}^{\hat{\mathcal{G}}(i r) \alpha}\right\}_{\alpha \geq 0}$ instead of $\left\{\mathrm{e}^{\hat{\mathcal{G}}(0) \alpha}=\mathrm{e}^{\hat{\mathcal{G}} \alpha}\right\}_{\alpha \geq 0}$ is that the continuous spectrum of $\hat{\mathcal{G}}$ (coming from the reservoir) is shifted to the left half plane. Indeed, in contrast to $\hat{\mathcal{G}}$, if $r>0$ is sufficiently large, the generator $\hat{\mathcal{G}}(i r)$ has discrete spectrum, as explained below. The effective atomic Lindbladian defined in (2.20) is related to Kato's perturbation theory at second order for the discrete spectrum of $\hat{\mathcal{G}}(i r)$ near the origin.

From now on, let $r \in\left(0, \mathrm{r}_{\max }\right)$. For $\lambda=\eta=0$, i.e., in absence of pump and atom-reservoir interaction, the discrete spectrum of the operator $\hat{\mathcal{G}}_{0}(i r)$ equals

$$
\sigma_{d}\left(\hat{\mathcal{G}}_{0}(i r)\right)=i\left(\varpi \mathbb{Z}+\sigma\left(i \mathfrak{L}_{\mathrm{at}}\right)\right)
$$

see (2.10) and (4.48). The full spectrum of $\hat{\mathcal{G}}_{0}(i r)$ is

$$
\sigma\left(\hat{\mathcal{G}}_{0}(i r)\right)=\sigma_{d}\left(\hat{\mathcal{G}}_{0}(i r)\right) \cup\{i \mathbb{R}-r \mathbb{N}\}
$$

In particular, there is a strictly positive gap between the discrete and essential spectra of $\hat{\mathcal{G}}_{0}(\mathrm{ir})$ :

$$
\operatorname{dist}\left(\sigma_{d}\left(\hat{\mathcal{G}}_{0}(i r)\right),\{i \mathbb{R}-r \mathbb{N}\}\right)=r>0 .
$$

For $r \in\left[0, \mathrm{r}_{\max }\right)$, let $\mathcal{J} \in \mathcal{B}(\hat{\mathcal{H}})$ and $\mathcal{I}(i r) \in \mathcal{B}(\hat{\mathcal{H}})$ be such that

$$
\hat{\mathcal{G}}(i r):=\hat{\mathcal{G}}_{0}(i r)+i \hat{V}(i r)=\hat{\mathcal{G}}_{0}(i r)+\eta \mathcal{J}+\lambda \mathcal{I}(i r)
$$


for any $\lambda, \eta \in \mathbb{R}$, see (4.51). I.e., $\eta \mathcal{J}:=\left.\hat{V}(i r)\right|_{\lambda=0}$ and $\lambda \mathcal{I}(i r):=\left.\hat{V}(i r)\right|_{\eta=0}$ are the interaction parts of $\hat{\mathcal{G}}(i r)$ respectively related to the pump and the atom-reservoir interaction. By Kato's perturbation theory for the discrete spectrum, if $|\lambda|,|\eta|$ are small, the deformed Howland generator $\hat{\mathcal{G}}(\mathrm{ir})$ has discrete spectrum.

Indeed, for $r \in\left(0, \mathrm{r}_{\max }\right)$, let $\mathfrak{r} \in(0,1 / 2) \cap(0, r)$ be such that

$$
\forall p \in \mathbb{Z}, \epsilon \in \sigma\left(i \mathfrak{L}_{\text {at }}\right): \quad \operatorname{dist}\left(i(p \varpi+\epsilon), \sigma\left(\hat{\mathcal{G}}_{0}(i r)\right) \backslash\{i(p \varpi+\epsilon)\}\right) \geq 2 \mathfrak{r}
$$

and, for $p \in \mathbb{Z}, \epsilon \in \sigma\left(i \mathfrak{L}_{\text {at }}\right)$, the contour $\gamma_{p, \epsilon}$ be defined by

$$
\gamma_{p, \epsilon}(y):=i(p \varpi+\epsilon)+\mathfrak{r} \mathrm{e}^{2 \pi i y} \in \mathbb{C}, \quad y \in[0,1]
$$

Then, for every $\eta \in \mathbb{R}, p \in \mathbb{Z}, \epsilon \in \sigma\left(i \mathfrak{L}_{\text {at }}\right)$, and sufficiently small $|\lambda|$, the operator

$$
\mathcal{P}_{p, \epsilon}^{(\lambda, \eta)}:=\frac{1}{2 \pi i} \oint_{\gamma_{p, \epsilon}}(\zeta-\hat{\mathcal{G}}(i r))^{-1} \mathrm{~d} \zeta
$$

is the well-known Riesz projection associated with $\hat{\mathcal{G}}(i r)$ and the discrete eigenvalue $i(p \varpi+\epsilon)$ of $\hat{\mathcal{G}}$ ( $(i r)$. Define also

$$
\overline{\mathcal{P}}_{p, \epsilon}^{(\lambda, \eta)}:=\mathbf{1}_{\hat{\mathcal{H}}}-\mathcal{P}_{p, \epsilon}^{(\lambda, \eta)}, \quad \lambda, \eta \in \mathbb{R}, p \in \mathbb{Z}, \epsilon \in \sigma\left(i \mathfrak{L}_{\text {at }}\right)
$$

If $\zeta \in \mathbb{C}$ is in the spectrum of $\hat{\mathcal{G}}_{0}(i r)$ and thus $\left(\zeta-\hat{\mathcal{G}}_{0}(i r)\right)$ is not a bijective map from $\operatorname{Dom}(\hat{\mathcal{G}} 0(i r))$ to $\hat{\mathcal{H}}$, the inverse $\left(\zeta-\hat{\mathcal{G}}_{0}(i r)\right)^{-1}$ will be understood below in the sense of multi-valued functions. In fact, observe that with this convention expressions of the form

$$
\overline{\mathcal{P}}_{p, \epsilon}^{(0,0)}\left(i(p \varpi+\epsilon)-\hat{\mathcal{G}}_{0}(i r)\right)^{-1} \overline{\mathcal{P}}_{p, \epsilon}^{(0,0)}
$$

with $p \in \mathbb{Z}, \epsilon \in \sigma\left(i \mathfrak{L}_{\text {at }}\right)$ define single-valued linear maps. Recall that $p \in \mathbb{Z}, \epsilon \in \sigma\left(i \mathfrak{L}_{\text {at }}\right)$ are eigenvalues of $\hat{\mathcal{G}}_{0}(i r)$ and thus $\left(i(p \varpi+\epsilon)-\hat{\mathcal{G}}_{0}(i r)\right)^{-1}$ is not single-valued in this case.

Lemma 4.12 (Perturbative expansions of the deformed Howland operator)

Let $r \in\left(0, \mathrm{r}_{\max }\right)$. For all $p \in \mathbb{Z}, \epsilon \in \sigma\left(i \mathfrak{L}_{\text {at }}\right)$ and sufficiently small $|\lambda|$,

$$
\begin{aligned}
\mathcal{P}_{p, \epsilon}^{(\lambda, \eta)} \hat{\mathcal{G}}(i r) \mathcal{P}_{p, \epsilon}^{(\lambda, \eta)}= & i(p \varpi+\epsilon) \mathcal{P}_{p, \epsilon}^{(0,0)}+\eta \mathcal{P}_{p, \epsilon}^{(0,0)} \mathcal{J} \mathcal{P}_{p, \epsilon}^{(0,0)} \\
& +\lambda^{2} \mathcal{P}_{p, \epsilon}^{(0,0)} \mathcal{I}(i r) \overline{\mathcal{P}}_{p, \epsilon}^{(0,0)}\left(i(p \varpi+\epsilon)-\hat{\mathcal{G}}_{0}(i r)\right)^{-1} \overline{\mathcal{P}}_{p, \epsilon}^{(0,0)} \mathcal{I}(i r) \mathcal{P}_{p, \epsilon}^{(0,0)} \\
& +\lambda^{3} \mathrm{R}
\end{aligned}
$$

with $\mathrm{R} \equiv \mathrm{R}^{(p, \epsilon, \lambda, \eta)}$ being an operator with norm $\|\mathrm{R}\|_{\mathcal{B}(\hat{\mathcal{H}})} \leq C$ for some finite constant $C \in \mathbb{R}^{+}$not depending on $p, \epsilon, \lambda$ and $\eta$.

Proof. We fix w.l.o.g. $p=\epsilon=0$. To simplify notation, in all the proof we denote by $\mathrm{R} \equiv \mathrm{R}^{(\lambda, \eta)}$ any operator with norm $\|\mathrm{R}\| \leq C$ for some fixed constant $C \in \mathbb{R}^{+}$not depending on $\lambda, \eta$. Note that the operator $\mathrm{R}$ does not need to be the same from one statement to another.

Assumption 3 yields, at small $|\lambda|,\|\hat{V}(i r)\| \leq C|\lambda|$, see (4.59). Hence, if $|\lambda|$ is sufficiently small then the resolvent $(\zeta-\hat{\mathcal{G}}(i r))^{-1}$ equals the absolutely convergent Neumann series

$$
(\zeta-\hat{\mathcal{G}}(i r))^{-1}=\sum_{n=0}^{\infty}\left(\zeta-\hat{\mathcal{G}}_{0}(i r)\right)^{-1}\left\{(\eta \mathcal{J}+\lambda \mathcal{I}(i r))\left(\zeta-\hat{\mathcal{G}}_{0}(i r)\right)^{-1}\right\}^{n}
$$

for all $\zeta \in \gamma_{0,0}$. By (4.62) and Assumption 3, it follows that

$$
\mathcal{P}_{0,0}^{(\lambda, \eta)}=\mathcal{P}_{0,0}^{(0,0)}+\eta P_{\mathrm{p}}^{(1)}+\lambda P_{\mathrm{at}, \mathcal{R}}^{(1)}+\lambda^{2} P_{\mathrm{at}, \mathcal{R}}^{(2)}+\lambda^{3} \mathrm{R},
$$


where

$$
\begin{aligned}
P_{\mathrm{p}}^{(1)} & :=\frac{1}{2 \pi i} \oint_{\gamma_{0,0}}\left(\zeta-\hat{\mathcal{G}}_{0}(i r)\right)^{-1} \mathcal{J}\left(\zeta-\hat{\mathcal{G}}_{0}(i r)\right)^{-1} \mathrm{~d} \zeta \\
P_{\mathrm{at}, \mathcal{R}}^{(1)} & :=\frac{1}{2 \pi i} \oint_{\gamma_{0,0}}\left(\zeta-\hat{\mathcal{G}}_{0}(i r)\right)^{-1} \mathcal{I}(i r)\left(\zeta-\hat{\mathcal{G}}_{0}(i r)\right)^{-1} \mathrm{~d} \zeta \\
P_{\mathrm{at}, \mathcal{R}}^{(2)} & :=\frac{1}{2 \pi i} \oint_{\gamma_{0,0}}\left(\zeta-\hat{\mathcal{G}}_{0}(i r)\right)^{-1}\left\{\mathcal{I}(i r)\left(\zeta-\hat{\mathcal{G}}_{0}(i r)\right)^{-1}\right\}^{2} \mathrm{~d} \zeta .
\end{aligned}
$$

We infer from (4.63)- (4.64) that

$$
\begin{aligned}
& \mathcal{P}_{0,0}^{(\lambda, \eta)} \hat{\mathcal{G}}(i r) \mathcal{P}_{0,0}^{(\lambda, \eta)} \\
= & \left(\mathcal{P}_{0,0}^{(0,0)}+\eta P_{\mathrm{p}}^{(1)}+\lambda P_{\mathrm{at}, \mathcal{R}}^{(1)}+\lambda^{2} P_{\mathrm{at}, \mathcal{R}}^{(2)}\right) \hat{\mathcal{G}}(i r)\left(\mathcal{P}_{0,0}^{(0,0)}+\eta P_{\mathrm{p}}^{(1)}+\lambda P_{\mathrm{at}, \mathcal{R}}^{(1)}+\lambda^{2} P_{\mathrm{at}, \mathcal{R}}^{(2)}\right) \\
& +\lambda^{3} \mathrm{R} .
\end{aligned}
$$

Note that, by Assumption 3, $\eta=O\left(\lambda^{2}\right)$. Thus, using the equality $\mathcal{P}_{0,0}^{(0,0)} \hat{\mathcal{G}}_{0}(i r)=0$ and (4.59) we obtain

$$
\begin{aligned}
\left(\mathcal{P}_{0,0}^{(0,0)}+\eta P_{\mathrm{p}}^{(1)}+\lambda P_{\mathrm{at}, \mathcal{R}}^{(1)}+\lambda^{2} P_{\mathrm{at}, \mathcal{R}}^{(2)}\right) \hat{\mathcal{G}}(i r)= & \mathcal{P}_{0,0}^{(0,0)}(\eta \mathcal{J}+\lambda \mathcal{I}(i r)) \\
& +\left(\eta P_{\mathrm{p}}^{(1)}+\lambda P_{\mathrm{at}, \mathcal{R}}^{(1)}+\lambda^{2} P_{\mathrm{at}, \mathcal{R}}^{(2)}\right) \hat{\mathcal{G}}_{0}(i r) \\
& +\lambda^{2} P_{\mathrm{at}, \mathcal{R}}^{(1)} \mathcal{I}(i r)+\lambda^{3} \mathrm{R} .
\end{aligned}
$$

Furthermore, from Assumption 2 and similar analyticity arguments as used in the proof of Theorem 4.11, one gets that

$$
\hat{\mathcal{G}}_{0}(i r) \mathcal{P}_{0,0}^{(0,0)}=0 \quad \text { and } \quad \mathcal{P}_{0,0}^{(0,0)} \mathcal{I}(\text { ir }) \mathcal{P}_{0,0}^{(0,0)}=0 .
$$

We deduce from (4.65) (4.67) that

$$
\begin{aligned}
\mathcal{P}_{0,0}^{(\lambda, \eta)} \hat{\mathcal{G}}(i r) \mathcal{P}_{0,0}^{(\lambda, \eta)}= & \eta \mathcal{P}_{0,0}^{(0,0)} \mathcal{J} \mathcal{P}_{0,0}^{(0,0)}+\lambda^{2} \mathcal{P}_{0,0}^{(0,0)} \mathcal{I}(i r) P_{\mathrm{at}, \mathcal{R}}^{(1)} \\
& +\lambda^{2} P_{\mathrm{at}, \mathcal{R}}^{(1)} \mathcal{I}(i r) \mathcal{P}_{0,0}^{(0,0)}+\lambda^{2} P_{\mathrm{at}, \mathcal{R}}^{(1)} \hat{\mathcal{G}}_{0}(i r) P_{\mathrm{at}, \mathcal{R}}^{(1)} \\
& +\lambda^{3} \mathrm{R}
\end{aligned}
$$

Since $\mathcal{P}_{0,0}^{(\lambda, \eta)}$ is a projection, obviously,

$$
\mathcal{P}_{0,0}^{(\lambda, \eta)} \hat{\mathcal{G}}(i r) \mathcal{P}_{0,0}^{(\lambda, \eta)}=\mathcal{P}_{0,0}^{(\lambda, \eta)}\left(\mathcal{P}_{0,0}^{(\lambda, \eta)} \hat{\mathcal{G}}(i r) \mathcal{P}_{0,0}^{(\lambda, \eta)}\right) \mathcal{P}_{0,0}^{(\lambda, \eta)}
$$

and, by (4.64) and (4.68),

$$
\begin{aligned}
\mathcal{P}_{0,0}^{(\lambda, \eta)} \hat{\mathcal{G}}(i r) \mathcal{P}_{0,0}^{(\lambda, \eta)}= & \eta \mathcal{P}_{0,0}^{(0,0)} \mathcal{J} \mathcal{P}_{0,0}^{(0,0)}+\lambda^{2} \mathcal{P}_{0,0}^{(0,0)} \mathcal{I}(i r) P_{\mathrm{at}, \mathcal{R}}^{(1)} \mathcal{P}_{0,0}^{(0,0)}+\lambda^{2} \mathcal{P}_{0,0}^{(0,0)} P_{\mathrm{at}, \mathcal{R}}^{(1)} \mathcal{I}(i r) \mathcal{P}_{0,0}^{(0,0)} \\
& +\lambda^{2} \mathcal{P}_{0,0}^{(0,0)} P_{\mathrm{at}, \mathcal{R}}^{(1)} \hat{\mathcal{G}}_{0}(i r) P_{\mathrm{at}, \mathcal{R}}^{(1)} \mathcal{P}_{0,0}^{(0,0)}+\lambda^{3} \mathrm{R}
\end{aligned}
$$

Now, using again $\mathcal{P}_{0,0}^{(0,0)} \hat{\mathcal{G}}_{0}(i r)=0$ and (4.67) we observe that

$$
\begin{aligned}
& \mathcal{P}_{0,0}^{(0,0)} P_{\mathrm{at}, \mathcal{R}}^{(1)} \hat{\mathcal{G}}_{0}(i r) P_{\mathrm{at}, \mathcal{R}}^{(1)} \mathcal{P}_{0,0}^{(0,0)} \\
= & \frac{1}{2 \pi i} \oint_{\gamma_{0,0}}\left\{\zeta_{1}^{-1} \mathcal{P}_{0,0}^{(0,0)} \mathcal{I}(i r)\left(\zeta_{1}-\hat{\mathcal{G}}_{0}(i r)\right)^{-1} \frac{1}{2 \pi i} \oint_{\gamma_{0,0}} \hat{\mathcal{G}}_{0}(i r)\left(\zeta_{2}-\hat{\mathcal{G}}_{0}(i r)\right)^{-1} \mathcal{I}(i r) \mathcal{P}_{0,0}^{(0,0)} \zeta_{2}^{-1} \mathrm{~d} \zeta_{2}\right\} \mathrm{d} \zeta_{1},
\end{aligned}
$$

while, by (4.67),

$$
\mathcal{I}(i r) \mathcal{P}_{0,0}^{(0,0)}=\left(\mathbf{1}_{\hat{\mathcal{H}}}-\mathcal{P}_{0,0}^{(0,0)}\right) \mathcal{I}(\text { ir }) \mathcal{P}_{0,0}^{(0,0)} \quad \text { and } \quad \mathcal{P}_{0,0}^{(0,0)} \mathcal{I}(\text { ir })=\mathcal{P}_{0,0}^{(0,0)} \mathcal{I}(\text { ir })\left(\mathbf{1}_{\hat{\mathcal{H}}}-\mathcal{P}_{0,0}^{(0,0)}\right)
$$


By analyticity of the map

$$
\zeta_{2} \mapsto \hat{\mathcal{G}}_{0}(i r)\left(\zeta_{2}-\hat{\mathcal{G}}_{0}(i r)\right)^{-1}\left(\mathbf{1}_{\hat{\mathcal{H}}}-\mathcal{P}_{0,0}^{(0,0)}\right) \mathcal{I}(i r) \mathcal{P}_{0,0}^{(0,0)},
$$

Equations (4.70) and (4.71) together imply that

$$
\begin{aligned}
& \mathcal{P}_{0,0}^{(0,0)} P_{\mathrm{at}, \mathcal{R}}^{(1)} \hat{\mathcal{G}}_{0}(i r) P_{\mathrm{at}, \mathcal{R}}^{(1)} \mathcal{P}_{0,0}^{(0,0)} \\
= & -\frac{1}{2 \pi i} \oint_{\gamma_{0,0}}\left\{\zeta^{-1} \mathcal{P}_{0,0}^{(0,0)} \mathcal{I}(i r)\left(\mathbf{1}_{\hat{\mathcal{H}}}-\mathcal{P}_{0,0}^{(0,0)}\right)\left(\zeta-\hat{\mathcal{G}}_{0}(i r)\right)^{-1}\left(\mathbf{1}_{\hat{\mathcal{H}}}-\mathcal{P}_{0,0}^{(0,0)}\right) \mathcal{I}(i r) \mathcal{P}_{0,0}^{(0,0)}\right\} \mathrm{d} \zeta .
\end{aligned}
$$

We also remark that (4.71) together with $\mathcal{P}_{0,0}^{(0,0)} \hat{\mathcal{G}}_{0}(i r)=0$ and (4.67) yields

$$
\begin{aligned}
\mathcal{P}_{0,0}^{(0,0)} P_{\mathrm{at}, \mathcal{R}}^{(1)} \mathcal{I}(i r) \mathcal{P}_{0,0}^{(0,0)} & =\frac{1}{2 \pi i} \oint_{\gamma_{0,0}}\left\{\zeta^{-1} \mathcal{P}_{0,0}^{(0,0)} \mathcal{I}(i r)\left(\mathbf{1}_{\hat{\mathcal{H}}}-\mathcal{P}_{0,0}^{(0,0)}\right)\left(\zeta-\hat{\mathcal{G}}_{0}(i r)\right)^{-1}\left(\mathbf{1}_{\hat{\mathcal{H}}}-\mathcal{P}_{0,0}^{(0,0)}\right) \mathcal{I}(i r) \mathcal{P}_{0,0}^{(0,0)}\right\} \mathrm{d} \zeta \\
& =\mathcal{P}_{0,0}^{(0,0)} \mathcal{I}(i r) P_{\mathrm{at}, \mathcal{R}}^{(1)} \mathcal{P}_{0,0}^{(0,0)}
\end{aligned}
$$

By analyticity of the map

$$
\zeta \mapsto \mathcal{P}_{0,0}^{(0,0)} \mathcal{I}(i r)\left(\mathbf{1}_{\hat{\mathcal{H}}}-\mathcal{P}_{0,0}^{(0,0)}\right)\left(\zeta-\hat{\mathcal{G}}_{0}(i r)\right)^{-1}\left(\mathbf{1}_{\hat{\mathcal{H}}}-\mathcal{P}_{0,0}^{(0,0)}\right) \mathcal{I}(i r) \mathcal{P}_{0,0}^{(0,0)},
$$

we then infer from (4.72)-(4.73) that

$$
\begin{aligned}
& \mathcal{P}_{0,0}^{(0,0)} \mathcal{I}(i r) P_{\mathrm{at}, \mathcal{R}}^{(1)} \mathcal{P}_{0,0}^{(0,0)}+\mathcal{P}_{0,0}^{(0,0)} P_{\mathrm{at}, \mathcal{R}}^{(1)} \mathcal{I}(i r) \mathcal{P}_{0,0}^{(0,0)}+\mathcal{P}_{0,0}^{(0,0)} P_{\mathrm{at}, \mathcal{R}}^{(1)} \hat{\mathcal{G}}_{0}(i r) P_{\mathrm{at}, \mathcal{R}}^{(1)} \mathcal{P}_{0,0}^{(0,0)} \\
= & \mathcal{P}_{0,0}^{(0,0)} \mathcal{I}(i r)\left(\mathbf{1}_{\hat{\mathcal{H}}}-\mathcal{P}_{0,0}^{(0,0)}\right)\left(i 0-\hat{\mathcal{G}}_{0}(i r)\right)^{-1}\left(\mathbf{1}_{\hat{\mathcal{H}}}-\mathcal{P}_{0,0}^{(0,0)}\right) \mathcal{I}(i r) \mathcal{P}_{0,0}^{(0,0)} .
\end{aligned}
$$

Using this and (4.69) we arrive at the assertion for $p=\epsilon=0$.

Up to some obvious changes in the above arguments, the general case with $p \in \mathbb{Z}$ and $\epsilon \in \sigma\left(i \mathfrak{L}_{\text {at }}\right)$ is proven in the same way. Note only that $\mathrm{R}$ is an operator with norm $\|\mathrm{R}\|_{\mathcal{B}(\hat{\mathcal{H}})} \leq C$ for some finite constant $C$ that does not depend on $p, \epsilon$ because of (4.60)- 4.61).

Similar to the atom-reservoir Lindbladian $\mathfrak{L}_{\mathcal{R}}^{(\varepsilon)} \in \mathcal{B}\left(\mathfrak{H}_{\text {at }}\right)(2.20)$, we define the operator $\mathcal{L}_{\mathcal{R}}^{(\varepsilon, r, p)} \in \mathcal{B}(\hat{\mathcal{H}})$ by

$$
\mathcal{L}_{\mathcal{R}}^{(\varepsilon, r, p)}:=\sum_{\epsilon \in \sigma\left(i \mathfrak{L}_{\mathrm{at}}\right)}\left[\mathcal{P}_{p, \epsilon}^{(0,0)} \mathcal{I}(i r) \overline{\mathcal{P}}_{p, \epsilon}^{(0,0)}\left(\varepsilon+i(p \varpi+\epsilon)-\hat{\mathcal{G}}_{0}(i r)\right)^{-1} \overline{\mathcal{P}}_{p, \epsilon}^{(0,0)} \mathcal{I}(i r) \mathcal{P}_{p, \epsilon}^{(0,0)}\right]^{*}
$$

for any $\varepsilon \in \mathbb{R}_{0}^{+}, r \in\left[0, \mathrm{r}_{\max }\right)$ and $p \in \mathbb{Z}$. This operator has the following important properties:

Lemma 4.13 (Properties of the operator $\mathcal{L}_{\mathcal{R}}^{(\varepsilon, r, p)}$ )

For any $p \in \mathbb{Z}, \varepsilon \in \mathbb{R}_{0}^{+}$and $r_{1}, r_{2} \in\left[0, r_{\max }\right)$,

$$
\mathcal{L}_{\mathcal{R}}^{\left(\varepsilon, r_{1}, p\right)}=\mathcal{L}_{\mathcal{R}}^{\left(\varepsilon, r_{2}, p\right)} .
$$

Moreover, in the sense of $\mathcal{B}(\hat{\mathcal{H}})$,

$$
\mathcal{L}_{\mathcal{R}}^{(p)}:=\mathcal{L}_{\mathcal{R}}^{(0,0, p)}=\lim _{\varepsilon \searrow 0} \mathcal{L}_{\mathcal{R}}^{(\varepsilon, 0, p)}
$$

Proof. Fix $p \in \mathbb{Z}$ and $\varepsilon \in \mathbb{R}_{0}^{+}$. By using similar analyticity arguments to those used in the proof of Theorem 4.11, one shows that $\left(\mathcal{L}_{\mathcal{R}}^{\left(\varepsilon, r_{1}, p\right)}\right)^{*}=\left(\mathcal{L}_{\mathcal{R}}^{\left(\varepsilon, r_{2}, p\right)}\right)^{*}$ for all $r_{1}, r_{2} \in\left[0, r_{\text {max }}\right)$, which is equivalent to the first assertion. Choose now $r \in\left(0, \mathrm{r}_{\max }\right)$. Then, by the first part of the lemma, $\mathcal{L}_{\mathcal{R}}^{(\varepsilon, 0, p)}=\mathcal{L}_{\mathcal{R}}^{(\varepsilon, r, p)}$ for all $\varepsilon \in \mathbb{R}_{0}^{+}$. For $r>0$,

$$
\lim _{\varepsilon \searrow 0} \overline{\mathcal{P}}_{p, \epsilon}^{(0,0)}\left(\varepsilon+i(p \varpi+\epsilon)-\hat{\mathcal{G}}_{0}(i r)\right)^{-1} \overline{\mathcal{P}}_{p, \epsilon}^{(0,0)}=\overline{\mathcal{P}}_{p, \epsilon}^{(0,0)}\left(i(p \varpi+\epsilon)-\hat{\mathcal{G}}_{0}(i r)\right)^{-1} \overline{\mathcal{P}}_{p, \epsilon}^{(0,0)},
$$

in the sense of $\mathcal{B}(\hat{\mathcal{H}})$ and the second assertion follows. 
We show below that $\mathcal{L}_{\mathcal{R}}^{(p)}$ acts - up to an equivalence transformation - as the Lindbladian $\mathfrak{L}_{\mathcal{R}}$ defined in (2.20).

Recall that the eigenspaces of the atomic Hamiltonian $H_{\text {at }} \in \mathcal{B}\left(\mathbb{C}^{d}\right)$ associated with the eigenvalues $E_{k}$, for $k \in\{1, \ldots, N\}$, and their dimensions are denoted by $\mathcal{H}_{k} \subset \mathbb{C}^{d}$ and $n_{k} \in \mathbb{N}$, respectively. By taking any arbitrary orthonormal basis $\left\{e_{n}^{(k)}\right\}_{n=1}^{n_{k}}$ of $\mathcal{H}_{k}$ for each $k \in\{1, \ldots, N\}$, we define the elements

$$
W_{(k, n)}^{\left(k^{\prime}, n^{\prime}\right)} \in \mathfrak{H}_{\mathrm{at}} \equiv \mathcal{B}\left(\mathbb{C}^{d}\right)
$$

for any $k, k^{\prime} \in\{1, \ldots, N\}, n \in\left\{1, \ldots, n_{k}\right\}$ and $n^{\prime} \in\left\{1, \ldots, n_{k^{\prime}}\right\}$ by the condition

$$
\forall k^{\prime \prime} \in\{1, \ldots, N\}, n^{\prime \prime} \in\left\{1, \ldots, n_{k^{\prime \prime}}\right\}: \quad W_{(k, n)}^{\left(k^{\prime}, n^{\prime}\right)}\left(e_{n^{\prime \prime}}^{\left(k^{\prime \prime}\right)}\right)=\delta_{n, n^{\prime \prime}} \delta_{k, k^{\prime \prime}} e_{n^{\prime}}^{\left(k^{\prime}\right)} .
$$

Then, for any $p \in \mathbb{Z}$ and $\epsilon \in \sigma\left(i \mathfrak{L}_{\text {at }}\right)$, straightforward computations show that

$$
\operatorname{ran}\left(\mathcal{P}_{p, \epsilon}^{(0,0)}\right)=\operatorname{span}\left\{\mathfrak{F}\left(\mathrm{e}^{i t \varpi m}\left(W_{(k, n)}^{\left(k^{\prime}, n^{\prime}\right)} \rho_{\text {at }}^{1 / 2}\right) \otimes \Omega_{\mathcal{R}}\right):\left(m, k, k^{\prime}\right) \in \mathbb{O}_{p, \epsilon}, n \in\left\{1, \ldots, n_{k}\right\}, n^{\prime} \in\left\{1, \ldots, n_{k^{\prime}}\right\}\right\}
$$

with

$$
\mathbb{O}_{p, \epsilon}:=\left\{\left(m, k, k^{\prime}\right) \in \mathbb{Z} \times\{1, \ldots, N\}^{2}: m \varpi+E_{k^{\prime}}-E_{k}=\varpi p+\epsilon\right\} .
$$

Recall that $\rho_{\text {at }} \in \mathcal{B}\left(\mathbb{C}^{d}\right)$ is the density matrix of the initial state of the atom. The range $\operatorname{ran}\left(\mathcal{P}_{p, \epsilon}^{(0,0)}\right)$ of the Riesz projection $\mathcal{P}_{p, \epsilon}^{(0,0)}$ does obviously not belong to the atomic space $\hat{\mathfrak{H}}_{\text {at }}(4.55)$. We can remove oscillating terms by using a unitary map $\mathrm{U}_{p, \epsilon}$ from $\operatorname{ran}\left(\mathcal{P}_{p, \epsilon}^{(0,0)}\right)$ to the atomic subspace

$$
\begin{array}{r}
\mathfrak{H}_{\mathrm{at}}^{(p, \epsilon)}:=\operatorname{span}\left\{W_{(k, n)}^{\left(k^{\prime}, n^{\prime}\right)} \rho_{\mathrm{at}}^{1 / 2}:\left(k, k^{\prime}\right) \in\{1, \ldots, N\}^{2}, n \in\left\{1, \ldots, n_{k}\right\}, n^{\prime} \in\left\{1, \ldots, n_{k^{\prime}}\right\},\right. \\
\left.\exists m \text { with }\left(m, k, k^{\prime}\right) \in \mathbb{O}_{p, \epsilon}\right\} \subset \mathfrak{H}_{\mathrm{at}}
\end{array}
$$

for $p \in \mathbb{Z}$ and $\epsilon \in \sigma\left(i \mathfrak{L}_{\text {at }}\right)$ as follows:

$$
\mathrm{U}_{p, \epsilon}\left(\mathfrak{F}\left(\mathrm{e}^{i t \varpi m}\left(W_{(k, n)}^{\left(k^{\prime}, n^{\prime}\right)} \rho_{\mathrm{at}}^{1 / 2}\right) \otimes \Omega_{\mathcal{R}}\right)\right):=W_{(k, n)}^{\left(k^{\prime}, n^{\prime}\right)} \rho_{\mathrm{at}}^{1 / 2} \in \mathfrak{H}_{\mathrm{at}}^{(p, \epsilon)}
$$

for any $k, k^{\prime} \in\{1, \ldots, N\}, n \in\left\{1, \ldots, n_{k}\right\}$ and $n^{\prime} \in\left\{1, \ldots, n_{k^{\prime}}\right\}$. Let $\mathfrak{S}$ be the bounded self-adjoint operator on $\mathcal{B}(\hat{\mathcal{H}})$ defined by

$$
(\mathfrak{S} \hat{f})(k):=\frac{1}{2}(\hat{f}(k+1)+\hat{f}(k-1)), \quad k \in \mathbb{Z} .
$$

Using the identification above of $\operatorname{ran}\left(\mathcal{P}_{p, \epsilon}^{(0,0)}\right)$ and $\mathfrak{H}_{\text {at }}^{(p, \epsilon)} \subset \mathfrak{H}_{\text {at }}$, we can establish a relation between the deformed Howland operator $\hat{\mathcal{G}}($ ir $)$ and the Lindbladians $\mathfrak{L}_{\mathrm{p}}(2.13)$ and $\mathfrak{L}_{\mathcal{R}}(2.20)$ via the adjoints $\mathfrak{L}_{\mathrm{p}}^{*}$, $\mathfrak{L}_{\mathcal{R}}^{*}$ w.r.t. the scalar product of $\mathfrak{H}_{\text {at }} \equiv \mathcal{B}\left(\mathbb{C}^{d}\right)$ defined by (2.5) and the operator $\mathfrak{Z}_{\text {at }}$ on $\mathfrak{H}_{\text {at }}$,

$$
\mathfrak{Z}_{\mathrm{at}}(A):=A \rho_{\mathrm{at}}^{1 / 2}, \quad A \in \mathfrak{H}_{\mathrm{Hat}} \equiv \mathcal{B}\left(\mathbb{C}^{d}\right) .
$$

Observe that, because $\rho_{\text {at }}$ is the density matrix of a faithful state, $\mathfrak{Z}_{\text {at }}$ has an inverse. By abuse of notation, $\mathfrak{L}_{\mathrm{p}}^{*}$ and $\mathfrak{L}_{\mathcal{R}}^{*}$ can be seen as operators defined on $\operatorname{ran}\left(\mathcal{P}_{p, \epsilon}^{(0,0)}\right)$ like in (4.33) or (4.49): Let

$$
\hat{\mathfrak{H}}_{\mathrm{im}}:=\left\{\hat{f}:=\{\hat{f}(k)\}_{k \in \mathbb{Z}}: \hat{f}(k) \in \mathfrak{H}_{\mathrm{at}} \otimes \Omega_{\mathcal{R}}, \sum_{k=-\infty}^{\infty}\|\hat{f}(k)\|_{\mathfrak{H}_{\mathrm{at}}}^{2}<\infty\right\} \subset \hat{\mathcal{H}} .
$$

For any linear operator $\mathfrak{L} \in \mathcal{B}\left(\hat{\mathfrak{H}}_{\text {im }}\right)$ and $\hat{f}:=\hat{u} \otimes \Omega_{\mathcal{R}} \in \hat{\mathfrak{H}}_{\text {im }}$, we define $\tilde{\mathfrak{L}} \equiv \mathfrak{L} \in \mathcal{B}\left(\hat{\mathfrak{H}}_{\text {im }}\right)$ by

$$
(\tilde{\mathfrak{L}} \hat{f})(k):=(\mathfrak{L} \hat{u}(k)) \otimes \Omega_{\mathcal{R}}, \quad k \in \mathbb{Z} .
$$

Note that $\operatorname{ran}\left(\mathcal{P}_{p, \epsilon}^{(0,0)}\right) \subset \hat{\mathfrak{H}}_{\text {im }}$ is an invariant subspace of $\mathfrak{L}_{\mathcal{R}}^{*} \equiv \tilde{\mathfrak{L}}_{\mathcal{R}}^{*}$ 
Theorem 4.14 (Effective microscopic dynamics)

For all $p \in \mathbb{Z}, \epsilon \in \sigma\left(i \mathfrak{L}_{\mathrm{at}}\right), A \in \mathfrak{H}_{\mathrm{at}}^{(p, \epsilon)}$ and sufficiently small $|\lambda|$,

$$
\begin{aligned}
\mathcal{P}_{p, \epsilon}^{(\lambda, \eta)} \hat{\mathcal{G}}(i r) \mathcal{P}_{p, \epsilon}^{(\lambda, \eta)} \mathrm{U}_{p, \epsilon}^{*}(A)= & i(p \varpi+\epsilon) \mathrm{U}_{p, \epsilon}^{*}(A)+\eta \mathcal{P}_{p, \epsilon}^{(0,0)} \mathfrak{S} \mathfrak{Z}_{\mathrm{at}} \mathfrak{L}_{\mathrm{p}}^{*} \mathfrak{Z}_{\mathrm{at}}^{-1} \mathrm{U}_{p, \epsilon}^{*}(A) \\
& +\lambda^{2} \mathfrak{Z}_{\mathrm{at}} \mathfrak{L}_{\mathcal{R}}^{*} \mathfrak{Z}_{\mathrm{at}}^{-1} \mathrm{U}_{p, \epsilon}^{*}(A)+\mathrm{RU}_{p, \epsilon}^{*}(A)
\end{aligned}
$$

where $\mathrm{R} \equiv \mathrm{R}^{(p, \epsilon, \lambda, \eta)}$ is an operator with norm $\|\mathrm{R}\|_{\mathcal{B}(\hat{\mathcal{H}})} \leq C|\lambda|^{3}$ for some finite constant $C \in \mathbb{R}^{+}$not depending on $p, \epsilon, \lambda, \eta$ and $A$.

Proof. By Lemma 4.13, if $A \in \mathfrak{H}_{\mathrm{at}}^{(p, \epsilon)}$ then

$$
\begin{aligned}
& \mathcal{P}_{p, \epsilon}^{(0,0)} \mathcal{I}(i r) \overline{\mathcal{P}}_{p, \epsilon}^{(0,0)}\left(i(p \varpi+\epsilon)-\hat{\mathcal{G}}_{0}(i r)\right)^{-1} \overline{\mathcal{P}}_{p, \epsilon}^{(0,0)} \mathcal{I}(i r) \mathcal{P}_{p, \epsilon}^{(0,0)} \mathrm{U}_{p, \epsilon}^{*}(A) \\
= & \left(\mathcal{L}_{\mathcal{R}}^{(0, r, p)}\right)^{*} \mathrm{U}_{p, \epsilon}^{*}(A)=\lim _{\varepsilon \searrow 0}\left(\mathcal{L}_{\mathcal{R}}^{(\varepsilon, 0, p)}\right)^{*} \mathrm{U}_{p, \epsilon}^{*}(A),
\end{aligned}
$$

see (4.74). Straightforward computations show that, for all $A \in \mathfrak{H}_{\mathrm{at}}^{(p, \epsilon)}$ and $\varepsilon \in \mathbb{R}^{+}$

$$
\mathcal{L}_{\mathcal{R}}^{(\varepsilon, 0, p) *} \mathrm{U}_{p, \epsilon}^{*}(A)=\mathfrak{Z}_{\text {at }} \mathfrak{L}_{\mathcal{R}}^{(\varepsilon) *} \mathfrak{Z}_{\text {at }}^{-1} \mathrm{U}_{p, \epsilon}^{*}(A)
$$

Hence, by the definition (2.20) of $\mathfrak{L}_{\mathcal{R}}$ together with (4.83),

$$
\mathcal{P}_{p, \epsilon}^{(0,0)} \mathcal{I}(i r) \overline{\mathcal{P}}_{p, \epsilon}^{(0,0)}\left(i(p \varpi+\epsilon)-\hat{\mathcal{G}}_{0}(i r)\right)^{-1} \overline{\mathcal{P}}_{p, \epsilon}^{(0,0)} \mathcal{I}(i r) \mathcal{P}_{p, \epsilon}^{(0,0)} \mathrm{U}_{p, \epsilon}^{*}(A)=\mathfrak{Z}_{\mathrm{at}} \mathfrak{L}_{\mathcal{R}}^{*} \mathfrak{Z}_{\mathrm{at}}^{-1} \mathrm{U}_{p, \epsilon}^{*}(A)
$$

for all $A \in \mathfrak{H}_{\mathrm{at}}^{(p, \epsilon)}$. Similarly, from straightforward computations, one gets that, for all $A \in \mathfrak{H}_{\mathrm{at}}^{(p, \epsilon)}$,

$$
\mathcal{P}_{p, \epsilon}^{(0,0)} \mathcal{J} \mathcal{P}_{p, \epsilon}^{(0,0)} \mathrm{U}_{p, \epsilon}^{*}(A)=\mathcal{P}_{p, \epsilon}^{(0,0)} \mathfrak{S} \mathfrak{Z}_{\mathrm{at}} \mathfrak{L}_{\mathrm{p}}^{*} \mathfrak{Z}_{\mathrm{at}}^{-1} \mathrm{U}_{p, \epsilon}^{*}(A)
$$

The theorem then follows from Lemma 4.12 combined with (4.83) and (4.84).

We are now in position to analyze the resonances of the Howland operator:

\section{Theorem 4.15 (Resonances of the Howland operator)}

Let $r \in\left(0, \mathrm{r}_{\max }\right)$. There are constants $\lambda_{0}, C_{1}, C_{2}, C_{3}, C_{4}, C_{5} \in \mathbb{R}^{+}$such that the following properties holds for all $|\lambda| \leq \lambda_{0}$ :

(i) For any $p \in \mathbb{Z}$, ip $\varpi$ is a non-degenerated eigenvalue of $\hat{\mathcal{G}}($ ir $)$ with eigenvector $\hat{f}_{p}$ defined by

$$
\hat{f}_{p}\left(p^{\prime}\right):=\delta_{p, p^{\prime}} \Omega, \quad p^{\prime} \in \mathbb{Z},
$$

where $\delta_{p, p^{\prime}}$ is the Kronecker symbol.

(ii) $\left\{i \mathbb{R}+\left(-C_{1} \lambda^{2}, \infty\right)\right\} \backslash i \varpi \mathbb{Z} \subset \varrho(\hat{\mathcal{G}}($ ir $))$. Here, $\varrho(\hat{\mathcal{G}}($ ir $))$ stands for the resolvent set of $\hat{\mathcal{G}}($ ir $)$.

(iii) The spectrum of $\hat{\mathcal{G}}($ ir $)$ in $i \mathbb{R}+\left(-C_{2}|\lambda|,-C_{1} \lambda^{2}\right)$ is discrete with algebraic multiplicity at most $d^{2}$, where $C_{2}>C_{1}|\lambda|$.

(iv) $i \mathbb{R}+\left(-C_{3},-C_{2}|\lambda|\right) \subset \varrho(\hat{\mathcal{G}}($ ir $))$, where $C_{3}>C_{2}|\lambda|$.

(v) For all $\zeta \in i \mathbb{R}+\left(-C_{3},-C_{4}\right)$, where $C_{3}>C_{4}$,

$$
\left\|(\zeta-\hat{\mathcal{G}}(i r))^{-1}\right\|_{\mathcal{B}(\hat{\mathcal{H}})} \leq C_{5}
$$

(vi) For any $D \in \mathbb{R}^{+}$, there is $C_{D} \in \mathbb{R}^{+}$such that

$$
\sup _{\operatorname{Re} \zeta>D}\left\|(\zeta-\hat{\mathcal{G}}(i r))^{-1}\right\|_{\mathcal{B}(\hat{\mathcal{H}})} \leq C_{D}
$$

Proof. (i) For $p_{1} \in \mathbb{Z}$, assume that $i p_{1} \varpi$ is an eigenvalue of $\hat{\mathcal{G}}(i r)$ with eigenvector $\hat{f}_{p_{1}} \in \hat{\mathcal{H}}$. Then, for $p_{2} \in \mathbb{Z}$, the vector $\hat{f}_{p_{2}}$ defined by

$$
\hat{f}_{p_{2}}(p):=\hat{f}_{p_{1}}\left(p-p_{2}+p_{1}\right), \quad p \in \mathbb{Z},
$$


is an eigenvector of $\hat{\mathcal{G}}(i r)$ with eigenvalue $i p_{2} \varpi$. In fact, the map $\hat{f}(p) \mapsto \hat{f}\left(p-p_{2}+p_{1}\right)$ is a unitary transformation on $\hat{\mathcal{H}}$, whose conjugation with $\hat{\mathcal{G}}(i r)$ is $\hat{\mathcal{G}}(i r)+i\left(p_{2}-p_{1}\right)$. Consequently, it suffices to prove that $\hat{\mathcal{G}}(i r) \hat{f}_{0}=0$ and 0 is a non-degenerated eigenvalue of $\hat{\mathcal{G}}(i r)$.

Remark that the vector $\hat{f}_{0}$ defined by (4.85) is an eigenvector of $\hat{\mathcal{G}}(0)$ with eigenvalue 0 . Using (4.57) (4.58) we then deduce that $\hat{\mathcal{G}}(\theta) \hat{f}_{0}=0$ for all $\theta \in \mathbb{R}$. Recall that $\{\hat{\mathcal{G}}(\theta)\}_{\theta \in \mathbb{S} \backslash \mathbb{R}}$ is of type A (cf. Lemma 4.9) and $\hat{f}_{0} \in \operatorname{Dom}(\hat{\mathcal{G}}(0))$. It follows that, for all $\hat{\psi} \in \hat{\mathcal{H}}$, the continuous function

$$
\left.g(\theta):=\langle\hat{\psi}, \hat{\mathcal{G}}(\theta)) \hat{f}_{0}\right\rangle_{\hat{\mathcal{H}}}, \quad \theta \in \mathbb{S},
$$

is analytic on $\mathbb{S} \backslash \mathbb{R}$ and is zero on the real line. We thus infer from the Schwarz reflection principle that $g=0$ on $\mathbb{S}$. In other words, $\hat{\mathcal{G}}(i r) \hat{f}_{0}=0 \in \hat{\mathcal{H}}$ for $r \in\left(0, \mathrm{r}_{\text {max }}\right)$ and so, the vectors $\hat{f}_{p}$ defined by (4.85) are, for any $p \in \mathbb{Z}$, eigenvector of $\hat{\mathcal{G}}($ ir $)$ with eigenvalue ip $\varpi$. It remains to prove that 0 is a non-degenerated eigenvalue.

By Assumption 3, $|\eta| \leq C \lambda^{2}$ for some fixed constant $C \in \mathbb{R}^{+}$and we can infer from (4.28), (4.29) and (4.31) that, at small $|\lambda|$,

$$
\forall \theta \in \mathbb{S}: \quad\|\hat{V}(\theta)\|_{\mathcal{B}(\hat{\mathcal{H}})} \leq C|\lambda| .
$$

By (4.24) and (4.48), we observe that 0 is also an isolated eigenvalue of the unperturbed Howland operator $\hat{\mathcal{G}}_{0}($ ir $)$ and by Kato's perturbation theory, it is an isolated eigenvalue of $\hat{\mathcal{G}}($ ir $)$ for sufficiently small $|\lambda|$ at any $r \in\left(0, \mathrm{r}_{\mathrm{max}}\right)$. However, 0 is still a degenerated eigenvalue of $\hat{\mathcal{G}}_{0}(i r)$. The interaction part of $\hat{\mathcal{G}}(i r)$ removes this degeneracy.

Indeed, by (4.48),

$$
\forall \hat{f} \in \operatorname{Dom}(\hat{\mathcal{G}}(i r)): \quad\langle\hat{f}, \hat{\mathcal{G}}(i r) \hat{f}\rangle_{\hat{\mathcal{H}}}=i\left\{\langle\hat{f}, \varpi k \hat{f}\rangle_{\hat{\mathcal{H}}}+\left\langle\hat{f}, L_{\mathfrak{g}} \hat{f}\right\rangle_{\hat{\mathcal{H}}}+\langle\hat{f}, \hat{V}(i r) \hat{f}\rangle\right\}-r\langle\hat{f}, \hat{N} \hat{f}\rangle_{\hat{\mathcal{H}}}
$$

The operators $\varpi k, L_{\mathfrak{g}}, \mathcal{J}$ (cf. (4.59) ) are self-adjoint and thus,

$$
i\left\{\langle\hat{f}, \varpi k \hat{f}\rangle_{\hat{\mathcal{H}}}+\left\langle\hat{f}, L_{\mathfrak{g}} \hat{f}\right\rangle_{\hat{\mathcal{H}}}+\langle\hat{f}, \mathcal{J} \hat{f}\rangle_{\hat{\mathcal{H}}}\right\} \in i \mathbb{R}
$$

Then, using Assumption 2, we conclude that, for some finite constant $C$ not depending on $\lambda$ and $r \in\left[0, r_{\max }\right)$,

$$
\operatorname{Re}\left\{\lambda\langle\hat{f}, \mathcal{I}(i r) \hat{f}\rangle-r\langle\hat{f}, \hat{N} \hat{f}\rangle_{\hat{\mathcal{H}}}\right\}<0
$$

whenever $r>C|\lambda|$ and $\hat{f}(k) \notin \mathfrak{H}_{\text {at }} \otimes \Omega_{\mathcal{R}}$ for some $k \in \mathbb{Z}$. This implies that, for fixed $r \in\left(0, r_{\max }\right)$ and sufficiently small $|\lambda|$, each eigenvector of $\hat{\mathcal{G}}(i r)$ associated with a purely imaginary eigenvalue $y \in i \mathbb{R}$ must be an element of the subspace $\hat{\mathfrak{H}}_{\text {im }}$ defined by (4.81).

Now, we need several definitions. Let

$$
\hat{\mathcal{D}}_{\mathrm{im}}:=\left\{\hat{f} \in \hat{\mathfrak{H}}_{\mathrm{im}}: \sum_{k=-\infty}^{\infty} k^{2}\|\hat{f}(k)\|_{\mathfrak{H}_{\text {at }}}^{2}<\infty\right\}
$$

and the (unbounded) operator $k_{\text {im }}$ be defined on $\hat{\mathcal{D}}_{\text {im }}$ by

$$
\left(k_{\mathrm{im}} \hat{f}\right)(k):=k \hat{f}(k), \quad k \in \mathbb{Z} .
$$

To simplify notation, we denote the operator $k_{\text {im }}$ by $k$. Define further the bounded operator $\hat{\mathcal{L}}_{\text {im }}^{(\lambda, \eta)} \in \mathcal{B}\left(\hat{\mathfrak{H}}_{\text {im }}\right)$ by

$$
\hat{\mathcal{L}}_{\mathrm{im}}^{(\lambda, \eta)}:=\mathfrak{Z}_{\mathrm{at}}\left(\mathfrak{L}_{\mathrm{at}}^{*}+\lambda^{2} \mathfrak{L}_{\mathcal{R}}^{*}\right) \mathfrak{Z}_{\mathrm{at}}^{-1}+\eta \mathfrak{S} \mathfrak{Z}_{\mathrm{at}} \mathfrak{L}_{\mathrm{p}}^{*} \mathfrak{Z}_{\mathrm{at}}^{-1}
$$

where $\mathfrak{L}_{\text {at }}^{*}, \mathfrak{L}_{\mathrm{p}}^{*}$ and $\mathfrak{L}_{\mathcal{R}}^{*}$ the adjoints of $\mathfrak{L}_{\text {at }}, \mathfrak{L}_{\mathrm{p}}$ and $\mathfrak{L}_{\mathcal{R}}$, respectively. See (2.8), (2.13), (2.21) and (4.80) as well as (4.81)-(4.82).

Similar to [1, Eq. (4.8)], define

$$
\hat{G}_{\mathrm{im}}^{(\lambda, \eta)}:=i \varpi k+\hat{\mathcal{L}}_{\mathrm{im}}^{(\lambda, \eta)}
$$


with dense domain $\hat{\mathcal{D}}_{\text {im }} \subset \hat{\mathfrak{H}}_{\text {im }}$. We denote by

$$
P_{0}^{(\lambda, \eta)}:=\frac{1}{2 \pi i} \oint_{\gamma_{0,0}}\left(z-\hat{G}_{\mathrm{im}}^{(\lambda, \eta)}\right)^{-1} \mathrm{~d} z
$$

the Riesz projection associated with the closed operator $\hat{G}_{\mathrm{im}}^{(\lambda, \eta)}$ defined by (4.89), for a sufficiently small enough parameter $\mathfrak{r}>0$ (cf. (4.60)-(4.61)).

From Theorem 4.14 ,

$$
\left\|\mathcal{P}_{0,0}^{(\lambda, \eta)} \hat{\mathcal{G}}(i r) \mathcal{P}_{0,0}^{(\lambda, \eta)} \mathcal{P}_{0,0}^{(0,0)}-\mathcal{P}_{0,0}^{(0,0)} \hat{G}_{\mathrm{im}}^{(\lambda, \eta)} \mathcal{P}_{0,0}^{(0,0)}\right\|_{\mathcal{B}(\hat{\mathfrak{H}})} \leq C|\lambda|^{3}
$$

Note that $\operatorname{ran}\left(\mathcal{P}_{0,0}^{(0,0)}\right) \subset \hat{\mathfrak{H}}_{\text {im }}$. Define

$$
\Lambda_{\mathrm{im}}^{(\lambda, \eta)}:=P_{0}^{(0,0)} \hat{G}_{\mathrm{im}}^{(\lambda, \eta)} P_{0}^{(0,0)}
$$

By observing that

$$
\mathcal{P}_{0,0}^{(0,0)}=P_{0}^{(0,0)} \mathcal{P}_{0,0}^{(0,0)},\left.\quad \mathcal{P}_{0,0}^{(0,0)}\right|_{\hat{\mathfrak{H}}_{\text {im }}}=\mathcal{P}_{0,0}^{(0,0)} P_{0}^{(0,0)}
$$

and using the bounds

$$
\left\|\mathcal{P}_{0,0}^{(\lambda, \eta)}-\mathcal{P}_{0,0}^{(0,0)}\right\|_{\mathcal{B}(\hat{\mathcal{H}})} \leq C|\lambda|, \quad\left\|\mathcal{P}_{0,0}^{(\lambda, \eta)} \hat{\mathcal{G}}(i r) \mathcal{P}_{0,0}^{(\lambda, \eta)}\right\|_{\mathcal{B}(\hat{\mathcal{H}})} \leq C \lambda^{2}
$$

we deduce that

$$
\left\|\mathcal{P}_{0,0}^{(\lambda, \eta)} \hat{\mathcal{G}}(i r) \mathcal{P}_{0,0}^{(\lambda, \eta)}-\mathcal{P}_{0,0}^{(0,0)} \Lambda_{\mathrm{im}}^{(\lambda, \eta)} \mathcal{P}_{0,0}^{(0,0)}\right\|_{\mathcal{B}(\hat{\mathcal{H}})} \leq C|\lambda|^{3}
$$

If Assumption 4 holds then, exactly like in [1, Lemma 6.3], for all $(\lambda, \eta) \in \mathbb{R} \times \mathbb{R}$,

$$
\min \left\{|\operatorname{Re}\{p\}|: p \in \sigma\left(\Lambda_{\mathrm{im}}^{(\lambda, \eta)}\right) \backslash\{0\}\right\} \geq C_{\varpi} \lambda^{2}
$$

with $C_{\varpi} \in \mathbb{R}^{+}$being a constant depending on $\varpi$ but not on $\lambda$, $\eta$. Similar to [1, Lemma 6.1] one proves, moreover, that 0 is a non-degenerated eigenvalue of $\Lambda_{\mathrm{im}}^{(\lambda, \eta)}$. Using this, the relations (4.91) and Kato's perturbation theory together with the bound (4.93), for small enough $|\lambda|, \hat{\mathcal{G}}($ ir $)$ has a non-degenerated eigenvalue $\varepsilon(\lambda, \eta)=\mathcal{O}\left(\lambda^{3}\right)$ with

$$
\min \{|\operatorname{Re}\{p\}|: p \in \sigma(\hat{\mathcal{G}}(i r)) \backslash\{\varepsilon(\lambda, \eta)\}\} \geq C_{\varpi} \lambda^{2} .
$$

[Lemma 5.4 applied to the pair of projections $\mathcal{P}_{0,0}^{(0,0)}$ and $\mathcal{P}_{0,0}^{(\lambda, \eta)}$ can be useful in this context.] As 0 is an eigenvalue of $\hat{\mathcal{G}}($ ir $)$, it follows that $\varepsilon(\lambda, \eta)=0$ and 0 is a non-degenerated eigenvalue of $\hat{\mathcal{G}}($ ir $)$, provided $|\lambda|$ is sufficiently small. As a consequence, the vectors $\hat{f}_{p}$ defined by (4.85) for $p \in \mathbb{Z}$ are eigenvectors of $\hat{\mathcal{G}}(i r)$ associated with the non-degenerate eigenvalues $i p \varpi$, for small enough $|\lambda|$.

(ii) As explained in the beginning of the proof, it suffices to prove this statement for the spectrum near 0 . Therefore, the assertion is a direct consequence of the estimate (4.94).

(iii) Recall that 0 is an isolated eigenvalue of the unperturbed Howland operator $\hat{\mathcal{G}}_{0}(i r)$ with algebraic multiplicity $n \in \mathbb{N}, n \leq d^{2}$. By Kato's perturbation theory and (4.87), there are at most $n$ eigenvalues of $\hat{\mathcal{G}}(i r)$ within a ball of radius $|\lambda|$ with algebraic multiplicity at most $d^{2}$. Therefore, we arrive at the third assertion by combining this observation with (i)-(ii).

(iv) and (v) are easy to verify for the case $V=0$ because the operator $\hat{\mathcal{G}}_{0}(i r)$ is equivalent to a normal operator with explicitly known spectrum. The general case is proved by using simple power expansion for the resolvents of $\hat{\mathcal{G}}($ ir $)$ as $\hat{V}$ is a bounded operator of order $\mathcal{O}(\lambda)$.

(vi) To prove the last assertion, use the fact that the eigenvalues ip $\varpi, p \in \mathbb{Z}$, are non-degenerated. By Kato's perturbation theory, its resolvent near such spectral points behaves in the limit $\zeta \rightarrow i p \varpi$ as

$$
\left\|(\zeta-i p \varpi-\hat{\mathcal{G}}(i r))^{-1}\right\|_{\mathcal{B}(\hat{\mathcal{H}})} \leq C|\zeta-i p \varpi|^{-1},
$$

where $C \in \mathbb{R}^{+}$is a constant not depending on $p \in \mathbb{Z}$. The uniformity of this last estimate is related to the fact that the spectral spaces associated with the eigenvalues $i p \varpi$ are all unitarily equivalent, see Equation (4.86). 


\subsection{Time-uniform approximations of $\left\{\mathrm{e}^{\alpha \hat{\mathcal{G}}(i r)}\right\}_{\alpha \geq 0}$}

Let $r \in\left(0, \mathrm{r}_{\text {max }}\right)$ (see Section 3.1). The strongly continuous one-parameter semigroup $\left\{\mathrm{e}^{\alpha \hat{\mathcal{G}}(i r)}\right\}_{\alpha \geq 0}$ can be represented as the inverse Laplace transform of the resolvent of $\hat{\mathcal{G}}(i r)$. Indeed, by Theorem 4.15 (vi) and the Gearhart-Prüss-Greiner Theorem [25, Chap. V, 1.11], for any $D \in \mathbb{R}^{+}$, there is $C_{D} \in \mathbb{R}^{+}$such that

$$
\forall \alpha \in \mathbb{R}_{0}^{+}: \quad\left\|\mathrm{e}^{\alpha \hat{\mathcal{G}}(i r)}\right\|_{\mathcal{B}(\hat{\mathcal{H}})} \leq C_{D} \mathrm{e}^{D \alpha},
$$

i.e., the growth bound of the semigroup $\left\{\mathrm{e}^{\alpha \hat{\mathcal{G}}(i r)}\right\}_{\alpha \geq 0}$ is zero. Hence, by Lemma 5.3 ,

$$
\mathrm{e}^{\alpha \hat{\mathcal{G}}(i r)} \hat{f}=\lim _{N \rightarrow \infty}\left\{\frac{1}{2 \pi i} \int_{w-i N}^{w+i N} \mathrm{e}^{\alpha \zeta}(\zeta-\hat{\mathcal{G}}(i r))^{-1} \hat{f} \mathrm{~d} \zeta\right\}
$$

for all $\hat{f} \in \operatorname{Dom}(\hat{\mathcal{G}}(i r)), w, \alpha \in \mathbb{R}^{+}$. Next, we modify the contour of integration to make Riesz projections appear.

To this end, define

$$
\tilde{\mathcal{P}}_{p}^{(\lambda, \eta)}:=\frac{1}{2 \pi i} \oint_{\tilde{\gamma}_{p}}(\zeta-\hat{\mathcal{G}}(i r))^{-1} \mathrm{~d} \zeta, \quad p \in \mathbb{Z},
$$

where, for any $p \in \mathbb{Z}$, the contour $\tilde{\gamma}_{p}$ is defined by

$$
\tilde{\gamma}_{p}(y):= \begin{cases}i(p \varpi+\mathfrak{r})+i(\varpi-2 \mathfrak{r}) y & \text { for } y \in[0,1] . \\ i((p+1) \varpi-\mathfrak{r})-C_{4}(y-1) & \text { for } y \in[1,2] . \\ -C_{4}+i((p+1) \varpi-\mathfrak{r})-i(\varpi-2 \mathfrak{r})(y-2) & \text { for } y \in[2,3] . \\ -C_{4}+i(p \varpi+\mathfrak{r})+C_{4}(y-3) & \text { for } y \in[3,4]\end{cases}
$$

Here, $\mathfrak{r} \in(0,1 / 2) \cap\left(0, r_{\max }\right)$ is a sufficiently small parameter, see (4.60)-(4.62), while $C_{4} \in \mathbb{R}^{+}$is the constant of Theorem 4.15 (v). For all $\hat{f} \in \operatorname{Dom}(\hat{\mathcal{G}}(i r)), w, \alpha \in \mathbb{R}^{+}$, and any negative real number $v \in\left(-C_{3},-C_{4}\right)$ (cf. Theorem 4.15) we now observe that, for $N \in \varpi \mathbb{N}+\mathfrak{r}$ and sufficiently small $|\lambda|$,

$$
\begin{aligned}
\frac{1}{2 \pi i} \int_{w-i N}^{w+i N} \mathrm{e}^{\alpha \zeta}(\zeta-\hat{\mathcal{G}}(i r))^{-1} \hat{f} \mathrm{~d} \zeta= & \sum_{p \in \mathbb{Z}:|p| \varpi<N} \mathrm{e}^{\alpha \mathcal{K}_{p}^{(\lambda, \eta)}} \mathcal{P}_{p, 0}^{(\lambda, \eta)} \hat{f} \\
& +\sum_{p \in \mathbb{Z}: p \varpi,(p+1) \varpi \in[-N, N]} \mathrm{e}^{\alpha \tilde{\mathcal{K}}_{p}^{(\lambda, \eta)}} \tilde{\mathcal{P}}_{p}^{(\lambda, \eta)} \hat{f} \\
& -\frac{1}{2 \pi i} \int_{w+i N}^{v+i N} \mathrm{e}^{\alpha \zeta}(\zeta-\hat{\mathcal{G}}(i r))^{-1} \hat{f} \mathrm{~d} \zeta \\
& -\frac{1}{2 \pi i} \int_{v+i N}^{v-i N} \mathrm{e}^{\alpha \zeta}(\zeta-\hat{\mathcal{G}}(i r))^{-1} \hat{f} \mathrm{~d} \zeta \\
& -\frac{1}{2 \pi i} \int_{v-i N}^{w-i N} \mathrm{e}^{\alpha \zeta}(\zeta-\hat{\mathcal{G}}(i r))^{-1} \hat{f} \mathrm{~d} \zeta,
\end{aligned}
$$

where

$$
\begin{array}{lll}
\mathcal{K}_{p}^{(\lambda, \eta)}:=\mathcal{P}_{p, 0}^{(\lambda, \eta)} \hat{\mathcal{G}}(i r) \mathcal{P}_{p, 0}^{(\lambda, \eta)}, & p \in \mathbb{Z}, \\
\tilde{\mathcal{K}}_{p}^{(\lambda, \eta)}:=\tilde{\mathcal{P}}_{p}^{(\lambda, \eta)} \hat{\mathcal{G}}(i r) \tilde{\mathcal{P}}_{p}^{(\lambda, \eta)}, & p \in \mathbb{Z} .
\end{array}
$$

We analyze in the three next lemmata each term of the right hand side (r.h.s.) of Equation (4.98), in the limit $N \rightarrow \infty$.

\section{Lemma 4.16}

For all $\hat{\psi}, \hat{\psi}^{\prime} \in \hat{\mathfrak{H}}_{\text {at }} \subset \operatorname{Dom}(\hat{\mathcal{G}}($ ir $)), w, \alpha \in \mathbb{R}^{+}$, and $v \in\left(-C_{3},-C_{4}\right)$,

$$
\lim _{N \rightarrow \infty} \int_{w \pm i N}^{v \pm i N} \mathrm{e}^{\alpha \zeta}(\zeta-\hat{\mathcal{G}}(i r))^{-1} \hat{\psi} \mathrm{d} \zeta=0
$$


while

$$
\limsup _{N \rightarrow \infty}\left|\left\langle\hat{\psi}, \int_{v+i N}^{v-i N} \mathrm{e}^{\alpha \zeta}(\zeta-\hat{\mathcal{G}}(i r))^{-1} \hat{\psi}^{\prime} \mathrm{d} \zeta\right\rangle_{\hat{\mathcal{H}}}\right| \leq C \lambda^{2} \mathrm{e}^{v \alpha}\|\hat{\psi}\|_{\hat{\mathcal{H}}}\left\|\hat{\psi}^{\prime}\right\|_{\hat{\mathcal{H}}}
$$

Proof. Using the equality

$$
\forall \hat{f} \in \operatorname{Dom}(\hat{\mathcal{G}}(i r)), \zeta \in \varrho(\hat{\mathcal{G}}(i r)): \quad(\zeta-\hat{\mathcal{G}}(i r))^{-1} \hat{f}=\zeta^{-1}\left((\zeta-\hat{\mathcal{G}}(i r))^{-1} \hat{\mathcal{G}}(i r) \hat{f}+\hat{f}\right)
$$

we deduce that, for any $\hat{\psi} \in \hat{\mathfrak{H}}_{\text {at }}$,

$$
\int_{w \pm i N}^{v \pm i N} \mathrm{e}^{\alpha \zeta}(\zeta-\hat{\mathcal{G}}(i r))^{-1} \hat{\psi} \mathrm{d} \zeta=\int_{w \pm i N}^{v \pm i N} \zeta^{-1} \mathrm{e}^{\alpha \zeta}(\zeta-\hat{\mathcal{G}}(i r))^{-1} \hat{\mathcal{G}}(i r) \hat{\psi} \mathrm{d} \zeta+\int_{w \pm i N}^{v \pm i N} \zeta^{-1} \mathrm{e}^{\alpha \zeta} \hat{\psi} \mathrm{d} \zeta
$$

Recall that $\varrho(\hat{\mathcal{G}}(i r))$ stands for the resolvent set of $\hat{\mathcal{G}}(i r)$. In the limit $N \rightarrow \infty$, both integrals in the r.h.s. of this last equality vanish.

We prove now the second inequality. Observe first that, for any $\zeta \in \varrho(\hat{\mathcal{G}}(i r))$ and sufficiently small $|\lambda|$,

$$
\begin{aligned}
(\zeta-\hat{\mathcal{G}}(i r))^{-1}= & \left(\zeta-\hat{\mathcal{G}}_{0}(i r)\right)^{-1}+\lambda\left(\zeta-\hat{\mathcal{G}}_{0}(i r)\right)^{-1} \mathcal{I}(i r)\left(\zeta-\hat{\mathcal{G}}_{0}(i r)\right)^{-1} \\
& +\eta\left(\zeta-\hat{\mathcal{G}}_{0}(i r)\right)^{-1} \mathcal{J}\left(\zeta-\hat{\mathcal{G}}_{0}(i r)\right)^{-1} \\
& +\left(\zeta-\hat{\mathcal{G}}_{0}(i r)\right)^{-1}(\eta \mathcal{J}+\lambda \mathcal{I}(i r))\left(\zeta-\hat{\mathcal{G}}_{0}(i r)\right)^{-1}(\eta \mathcal{J}+\lambda \mathcal{I}(i r))(\zeta-\hat{\mathcal{G}}(i r))^{-1}
\end{aligned}
$$

Because of Assumption 2 for all $\hat{\psi}, \hat{\psi}^{\prime} \in \hat{\mathfrak{H}}_{\text {at }}, \zeta \in \varrho(\hat{\mathcal{G}}(i r))$ and sufficiently small $|\lambda|$,

$$
\left\langle\hat{\psi},\left(\zeta-\hat{\mathcal{G}}_{0}(i r)\right)^{-1} \mathcal{I}(i r)\left(\zeta-\hat{\mathcal{G}}_{0}(i r)\right)^{-1} \hat{\psi}^{\prime}\right\rangle_{\hat{\mathcal{H}}}=0,
$$

while, by using (4.75),

$$
\begin{aligned}
& \lim _{N \rightarrow \infty}\left\langle\hat{\psi}, \int_{v+i N}^{v-i N}\left(\zeta-\hat{\mathcal{G}}_{0}(i r)\right)^{-1} \hat{\psi}^{\prime} \mathrm{d} \zeta\right\rangle_{\hat{\mathcal{H}}} \\
= & \sum_{(n, k),\left(n^{\prime}, k^{\prime}\right)}\left\langle\hat{\psi}, \mathfrak{F}\left(\left(W_{(k, n)}^{\left(k^{\prime}, n^{\prime}\right)} \rho_{\mathrm{at}}^{1 / 2}\right) \otimes \Omega_{\mathcal{R}}\right)\right\rangle_{\hat{\mathcal{H}}}\left\langle\mathfrak{F}\left(\left(W_{(k, n)}^{\left(k^{\prime}, n^{\prime}\right)} \rho_{\mathrm{at}}^{1 / 2}\right) \otimes \Omega_{\mathcal{R}}\right), \hat{\psi}^{\prime}\right\rangle_{\hat{\mathcal{H}}} \\
& \times \lim _{N \rightarrow \infty} \int_{v+i N}^{v-i N} \mathrm{e}^{\alpha \zeta}\left(\zeta-i\left(E_{k^{\prime}}-E_{k}\right)\right)^{-1} \mathrm{~d} \zeta \\
= & 0
\end{aligned}
$$

for all $\alpha \in \mathbb{R}^{+}$and $v<0$. Clearly, for $\zeta \in v+i \mathbb{R}$ and $\hat{\psi}^{\prime} \in \hat{\mathfrak{H}}_{\mathrm{at}}$,

$$
\left\|\left(\zeta-\hat{\mathcal{G}}_{0}(i r)\right)^{-1} \mathcal{J}\left(\zeta-\hat{\mathcal{G}}_{0}(i r)\right)^{-1} \hat{\psi}^{\prime}\right\|_{\hat{\mathcal{H}}} \leq \frac{C}{|\zeta|^{2}}\left\|\hat{\psi}^{\prime}\right\|_{\hat{\mathcal{H}}}
$$

with $C \in \mathbb{R}^{+}$being some finite constant that only depends on $v$. Thus, using Assumption 3 ,

$$
\left|\left\langle\hat{\psi}, \int_{v+i N}^{v-i N} \mathrm{e}^{\alpha \zeta} \eta\left(\zeta-\hat{\mathcal{G}}_{0}(i r)\right)^{-1} \mathcal{J}\left(\zeta-\hat{\mathcal{G}}_{0}(i r)\right)^{-1} \hat{\psi}^{\prime} \mathrm{d} \zeta\right\rangle_{\hat{\mathcal{H}}}\right| \leq C \lambda^{2} \mathrm{e}^{\alpha v}\|\hat{\psi}\|_{\hat{\mathcal{H}}}\left\|\hat{\psi}^{\prime}\right\|_{\hat{\mathcal{H}}}
$$

for some constant $C \in \mathbb{R}^{+}$depending only on $v$.

Again by Assumption 3 note that, for all $\zeta \in v+i \mathbb{R}$ and $\hat{\psi} \in \hat{\mathfrak{H}}_{\text {at }}$,

$$
\left\|\left[\left(\zeta-\hat{\mathcal{G}}_{0}(i r)\right)^{-1}(\eta \mathcal{J}+\lambda \mathcal{I}(i r))\left(\zeta-\hat{\mathcal{G}}_{0}(i r)\right)^{-1}(\eta \mathcal{J}+\lambda \mathcal{I}(i r))\right]^{*} \hat{\psi}\right\|_{\hat{\mathcal{H}}} \leq \frac{C \lambda^{2}}{|\zeta|}\|\hat{\psi}\|_{\hat{\mathcal{H}}}
$$

with $C \in \mathbb{R}^{+}$being some finite constant only depending on $v$. On the other hand, by using Equality (4.101) together with Theorem $4.15(\mathrm{v})$, we obtain

$$
\left\|(\zeta-\hat{\mathcal{G}}(i r))^{-1} \hat{\psi}^{\prime}\right\|_{\hat{\mathcal{H}}} \leq \frac{C}{|\zeta|}\left\|\hat{\psi}^{\prime}\right\|_{\hat{\mathcal{H}}}
$$


for some constant $C \in \mathbb{R}^{+}$that does not depend on $\zeta \in v+i \mathbb{R}$ and $\hat{\psi}^{\prime} \in \hat{\mathfrak{H}}_{\text {at }}$. Hence,

$$
\begin{aligned}
& \left|\left\langle\hat{\psi}, \int_{v+i N}^{v-i N} \mathrm{e}^{\alpha \zeta}\left(\zeta-\hat{\mathcal{G}}_{0}(i r)\right)^{-1}(\eta \mathcal{J}+\lambda \mathcal{I}(i r))\left(\zeta-\hat{\mathcal{G}}_{0}(i r)\right)^{-1}(\eta \mathcal{J}+\lambda \mathcal{I}(i r))(\zeta-\hat{\mathcal{G}}(i r))^{-1} \hat{\psi}^{\prime} \mathrm{d} \zeta\right\rangle_{\hat{\mathcal{H}}}\right| \\
\leq & C \lambda^{2} \mathrm{e}^{\alpha v}\|\hat{\psi}\|_{\hat{\mathcal{H}}}\left\|\hat{\psi}^{\prime}\right\|_{\hat{\mathcal{H}}}
\end{aligned}
$$

for all $\hat{\psi}, \hat{\psi}^{\prime} \in \hat{\mathfrak{H}}_{\text {at }}, \alpha \in \mathbb{R}^{+}$and some constant $C \in \mathbb{R}^{+}$that does not depend on $\hat{\psi}, \hat{\psi}^{\prime}$ and $\alpha$.

\section{Lemma 4.17}

There is a constant $C \in \mathbb{R}^{+}$such that, for all $\hat{\psi} \in \hat{\mathfrak{H}}_{\text {at }} \subset \hat{\mathcal{H}}$,

$$
\begin{array}{ll}
\forall p \in \mathbb{Z} \backslash\{0\} & : \quad\left\|\mathcal{P}_{p, 0}^{(\lambda, \eta)} \hat{\psi}\right\|_{\hat{\mathcal{H}}} \leq C p^{-2} \varpi^{-2}\|\hat{\psi}\|_{\hat{\mathcal{H}}}, \\
\forall p \in \mathbb{Z} \backslash\{0,-1\} & : \quad\left\|\tilde{\mathcal{P}}_{p}^{(\lambda, \eta)} \hat{\psi}\right\|_{\hat{\mathcal{H}}} \leq C p^{-2} \varpi^{-2}\|\hat{\psi}\|_{\hat{\mathcal{H}}} .
\end{array}
$$

Moreover, if $\hat{\psi} \in \mathfrak{F}\left(\mathfrak{D} \otimes \Omega_{\mathcal{R}}\right)$ then

$$
\forall p \in \mathbb{Z}: \quad\left\|\tilde{\mathcal{P}}_{p}^{(\lambda, \eta)} \hat{\psi}\right\|_{\hat{\mathcal{H}}} \leq C|\lambda|\left(\frac{1}{\varpi^{2} p^{2}+1}+\frac{1}{\varpi^{2}(p+1)^{2}+1}\right)\|\hat{\psi}\|_{\hat{\mathcal{H}}} .
$$

Proof. Using two times Equality (4.101), for any $p \in \mathbb{Z} \backslash\{0\}$ and $\hat{\psi} \in \hat{\mathfrak{H}}_{\text {at }} \subset \operatorname{Dom}\left((\hat{\mathcal{G}}(i r))^{2}\right)$, in the definition (4.62) of the Riesz projection $\mathcal{P}_{p, 0}^{(\lambda, \eta)}$, we obtain that

$$
\begin{aligned}
\mathcal{P}_{p, 0}^{(\lambda, \eta)} \hat{\psi} & =\frac{1}{2 \pi i} \oint_{\gamma_{p, 0}} \zeta^{-2}(\zeta-\hat{\mathcal{G}}(i r))^{-1}(\hat{\mathcal{G}}(i r))^{2} \hat{\psi} \mathrm{d} \zeta+\frac{1}{2 \pi i} \oint_{\gamma_{p, 0}} \zeta^{-2} \hat{\mathcal{G}}(i r) \hat{\psi} \mathrm{d} \zeta+\frac{1}{2 \pi i} \oint_{\gamma_{p, 0}} \zeta^{-1} \hat{\psi} \mathrm{d} \zeta \\
& =\frac{1}{2 \pi i} \oint_{\gamma_{p, 0}} \zeta^{-2}(\zeta-\hat{\mathcal{G}}(i r))^{-1}(\hat{\mathcal{G}}(i r))^{2} \hat{\psi} \mathrm{d} \zeta,
\end{aligned}
$$

by analyticity. There is $C \in \mathbb{R}^{+}$such that, for $p \in \mathbb{Z} \backslash\{0\}, \zeta \in \gamma_{p, 0}$ and sufficiently small $|\lambda|$,

$$
\left\|(\zeta-\hat{\mathcal{G}}(i r))^{-1}\right\|_{\mathcal{B}(\hat{\mathcal{H}})} \leq C \quad \text { and } \quad\left\|(\hat{\mathcal{G}}(i r))^{2} \hat{\psi}\right\|_{\hat{\mathcal{H}}} \leq C\|\hat{\psi}\|_{\hat{\mathcal{H}}},
$$

see (4.35) and (4.51), while

$$
|\zeta|^{-2} \leq C p^{-2} \varpi^{-2}
$$

We thus arrive at the assertion

$$
\left\|\mathcal{P}_{p, 0}^{(\lambda, \eta)} \hat{\psi}\right\|_{\hat{\mathcal{H}}} \leq C p^{-2} \varpi^{-2}\|\hat{\psi}\|_{\hat{\mathcal{H}}}
$$

with a constant $C \in \mathbb{R}^{+}$not depending on $p \in \mathbb{Z} \backslash\{0\}$.

To prove the second inequality, we proceed in the same way by using the fact that

$$
\left\|(\zeta-\hat{\mathcal{G}}(i r))^{-1}\right\|_{\mathcal{B}(\hat{\mathcal{H}})} \leq C
$$

for all $p \in \mathbb{Z}, \zeta \in \tilde{\gamma}_{p}$ and sufficiently small $|\lambda|$, by Theorem 4.15 Note also that (4.102) also holds for all $p \in \mathbb{Z} \backslash\{0,-1\}$ and $\zeta \in \tilde{\gamma}_{p}$.

To prove the last assertion, observe that if $\hat{\psi} \in \mathfrak{F}\left(\mathfrak{D} \otimes \Omega_{\mathcal{R}}\right)$ then

$$
\left\|(\hat{\mathcal{G}}(i r))^{2} \hat{\psi}\right\|_{\hat{\mathcal{H}}} \leq C|\lambda|\|\hat{\psi}\|_{\hat{\mathcal{H}}}
$$

for some constant $C \in \mathbb{R}^{+}$.

\section{Lemma 4.18}

There is a constant $C \in \mathbb{R}^{+}$such that, for all $p \in \mathbb{Z}$ and $\alpha \in \mathbb{R}_{0}^{+}$,

$$
\left\|\mathrm{e}^{\alpha \mathcal{K}_{p}}\right\|_{\mathcal{B}(\hat{\mathcal{H}})} \leq C \quad \text { and } \quad\left\|\mathrm{e}^{\alpha \tilde{\mathcal{K}}_{p}}\right\|_{\mathcal{B}(\hat{\mathcal{H}})} \leq C
$$


Proof. By Kato's perturbation theory and Assumption 3, which implies (4.87), the operator

$$
\mathcal{R}_{p}^{(\lambda, \eta)}:=\left(\mathcal{P}_{p, 0}^{(0,0)}-\mathcal{P}_{p, 0}^{(\lambda, \eta)}\right)^{2}, \quad p \in \mathbb{Z}
$$

is bounded by

$$
\left\|\mathcal{R}_{p}^{(\lambda, \eta)}\right\|_{\mathcal{B}(\hat{\mathcal{H}})} \leq C \lambda^{2}<1
$$

for $p \in \mathbb{Z}$, sufficiently small $|\lambda|$ and some constant $C \in \mathbb{R}^{+}$not depending on $p, \lambda$ (cf. (4.60)-(4.61)). Therefore, we can infer from Lemma 5.4 for $P=\mathcal{P}_{p, 0}^{(0,0)}$ and $Q=\mathcal{P}_{p, 0}^{(\lambda, \eta)}$ that there are two invertible, bounded operators

$$
U_{p}^{(\lambda, \eta)}:=\left(\mathcal{P}_{p, 0}^{(\lambda, \eta)} \mathcal{P}_{p, 0}^{(0,0)}+\left(1-\mathcal{P}_{p, 0}^{(\lambda, \eta)}\right)\left(1-\mathcal{P}_{p, 0}^{(0,0)}\right)\right)\left(1-\mathcal{R}_{p}^{(\lambda, \eta)}\right)^{-1 / 2}
$$

and

$$
V_{p}^{(\lambda, \eta)}:=\left(\mathcal{P}_{p, 0}^{(0,0)} \mathcal{P}_{p, 0}^{(\lambda, \eta)}+\left(1-\mathcal{P}_{p, 0}^{(0,0)}\right)\left(1-\mathcal{P}_{p, 0}^{(\lambda, \eta)}\right)\right)\left(1-\mathcal{R}_{p}^{(\lambda, \eta)}\right)^{-1 / 2}=\left(U_{p}^{(\lambda, \eta)}\right)^{-1}
$$

such that

$$
\mathcal{P}_{p, 0}^{(\lambda, \eta)}=U_{p}^{(\lambda, \eta)} \mathcal{P}_{p, 0}^{(0,0)} V_{p}^{(\lambda, \eta)}
$$

By Kato's perturbation theory and (4.87), there is $C \in \mathbb{R}^{+}$such that, for all $p \in \mathbb{Z}$ and sufficiently small $|\lambda|$,

$$
\left\|\mathcal{P}_{p, 0}^{(\lambda, \eta)}-\mathcal{P}_{p, 0}^{(0,0)}\right\|_{\mathcal{B}(\hat{\mathcal{H}})} \leq C|\lambda|
$$

Combined with (4.103), $\left\|\mathcal{P}_{p, 0}^{(0,0)}\right\|=1$ and the absolutely convergent binomial series

$$
\left(1-\mathcal{R}_{p}^{(\lambda, \eta)}\right)^{-1 / 2}=\mathbf{1}_{\hat{\mathcal{H}}}+\sum_{n=1}^{\infty}\left(\begin{array}{c}
-1 / 2 \\
n
\end{array}\right)\left(-\mathcal{R}_{p}^{(\lambda, \eta)}\right)^{n},
$$

Inequality (4.104) implies that

$$
\begin{aligned}
\left\|U_{p}^{(\lambda, \eta)} \mathcal{P}_{p, 0}^{(0,0)}-\mathcal{P}_{p, 0}^{(0,0)}\right\|_{\mathcal{B}(\hat{\mathcal{H}})} \leq C|\lambda|, \\
\left\|\mathcal{P}_{p, 0}^{(0,0)} V_{p}^{(\lambda, \eta)}-\mathcal{P}_{p, 0}^{(0,0)}\right\|_{\mathcal{B}(\hat{\mathcal{H}})} \leq C|\lambda|,
\end{aligned}
$$

for some constant $C \in \mathbb{R}^{+}$not depending on $p, \lambda(|\lambda|$ sufficiently small). By (4.99), it follows that

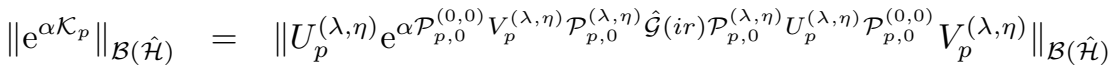

$$
\begin{aligned}
& \leq C\left\|\mathrm{e}^{\alpha \mathcal{P}_{p, 0}^{(0,0)} V_{p}^{(\lambda, \eta)} \mathcal{P}_{p, 0}^{(\lambda, \eta)} \hat{\mathcal{G}}(i r) \mathcal{P}_{p, 0}^{(\lambda, \eta)} U_{p}^{(\lambda, \eta)} \mathcal{P}_{p, 0}^{(0,0)}}\right\|_{\mathcal{B}(\hat{\mathcal{H}})}
\end{aligned}
$$

for all $p \in \mathbb{Z}$ and sufficiently small $|\lambda|$, with some constant $C \in \mathbb{R}^{+}$not depending on $p, \lambda$. Meanwhile, by using (4.105) - (4.106) as well as the Neumann series (4.63) and (4.67) extended to all $p \in \mathbb{Z}$ as it is done in Lemma 4.12 one verifies that

$$
\begin{aligned}
& \left\|\mathcal{P}_{p, 0}^{(0,0)} V_{p}^{(\lambda, \eta)} \mathcal{P}_{p, 0}^{(\lambda, \eta)} \hat{\mathcal{G}}(i r) \mathcal{P}_{p, 0}^{(\lambda, \eta)} U_{p}^{(\lambda, \eta)} \mathcal{P}_{p, 0}^{(0,0)}-\mathcal{P}_{p, 0}^{(0,0)} \mathcal{P}_{p, 0}^{(\lambda, \eta)} \hat{\mathcal{G}}(i r) \mathcal{P}_{p, 0}^{(\lambda, \eta)} \mathcal{P}_{p, 0}^{(0,0)}\right\|_{\mathcal{B}(\hat{\mathcal{H}})} \\
\leq & C|\lambda|^{3}
\end{aligned}
$$

for some finite constant $C \in \mathbb{R}^{+}$not depending on $p, \lambda, \eta$. Using the operator $\mathfrak{Z}_{\text {at }} \in \mathcal{B}\left(\mathfrak{H}_{\text {at }}\right)$ (4.80) and Theorem 4.14, it follows from (4.107) that, for all $p \in \mathbb{Z}, A \in \hat{\mathcal{H}}$ and sufficiently small $|\lambda|$,

$$
\begin{aligned}
\mathcal{P}_{p, 0}^{(0,0)} V_{p}^{(\lambda, \eta)}\left(\mathcal{P}_{p, 0}^{(\lambda, \eta)} \hat{\mathcal{G}}(i r) \mathcal{P}_{p, 0}^{(\lambda, \eta)}\right) U_{p}^{(\lambda, \eta)} \mathcal{P}_{p, 0}^{(0,0)}(A)= & i p \varpi \mathcal{P}_{p, 0}^{(0,0)}(A) \\
& +\eta \mathcal{P}_{p, 0}^{(0,0)} \mathfrak{S} \mathfrak{Z}_{\mathrm{at}} \mathfrak{L}_{\mathrm{p}}^{*} \mathfrak{Z}_{\mathrm{at}}^{-1} \mathcal{P}_{p, 0}^{(0,0)}(A) \\
& +\lambda^{2} \mathfrak{Z}_{\mathrm{at}} \mathfrak{L}_{\mathcal{R}}^{*} \mathfrak{Z}_{\mathrm{at}}^{-1} \mathcal{P}_{p, 0}^{(0,0)}(A) \\
& +\mathcal{P}_{p, 0}^{(0,0)} \mathrm{R} \mathcal{P}_{p, 0}^{(0,0)}(A)
\end{aligned}
$$


with $\mathrm{R} \equiv \mathrm{R}^{(p, \lambda, \eta)}$ being an operator with norm $\|\mathrm{R}\|_{\mathcal{B}(\hat{\mathcal{H}})} \leq C|\lambda|^{3}$ for some finite constant $C \in \mathbb{R}^{+}$not depending on $p, \lambda, \eta$ and $A$. Hence, since, by (4.79),

$$
\mathrm{U}_{p, 0} \mathcal{P}_{p, 0}^{(0,0)} \mathfrak{S} \mathfrak{Z}_{\mathrm{at}} \mathfrak{L}_{\mathrm{p}}^{*} \mathfrak{Z}_{\mathrm{at}}^{-1} \mathcal{P}_{p, 0}^{(0,0)} \mathrm{U}_{p, 0}^{*}=\left.\frac{1}{2} \mathfrak{Z}_{\mathrm{at}} \mathfrak{L}_{\mathrm{p}}^{*} \mathfrak{Z}_{\mathrm{at}}^{-1}\right|_{\mathfrak{H}_{\mathrm{at}}^{(p, 0)}}, \quad \mathrm{U}_{p, 0} \mathfrak{Z}_{\mathrm{at}} \mathfrak{L}_{\mathcal{R}}^{*} \mathfrak{Z}_{\mathrm{at}}^{-1} \mathrm{U}_{p, 0}^{*}=\left.\mathfrak{Z}_{\mathrm{at}} \mathfrak{L}_{\mathcal{R}}^{*} \mathfrak{Z}_{\mathrm{at}}^{-1}\right|_{\mathfrak{H}_{\mathrm{at}}^{(p, 0)}}
$$

(see (4.80) ), it suffices to bound the group on $\mathfrak{H}_{\mathrm{at}}^{(p, 0)}$ (4.77) generated by

$$
\mathcal{M}_{p}:=i p \varpi+\frac{\eta}{2} \mathfrak{L}_{\mathrm{p}}^{*}+\lambda^{2} \mathfrak{L}_{\mathcal{R}}^{*}+\Theta_{p}
$$

with

$$
\Theta_{p}:=\mathfrak{Z}_{\mathrm{at}}^{-1} \mathrm{U}_{p, 0} \mathcal{P}_{p, 0}^{(0,0)} \mathrm{R} \mathcal{P}_{p, 0}^{(0,0)} \mathrm{U}_{p, 0}^{*} \mathfrak{Z}_{\text {at }} .
$$

Note that $i \varpi p$ is a non-degenerated eigenvalue of $\mathcal{M}_{p}$ with associated Riesz projection $P$ satisfying $\|P\|_{\mathcal{B}\left(\mathfrak{H}_{\text {at }}^{(p, 0)}\right)} \leq 2$ for sufficiently small $|\lambda|$. Hence,

$$
\mathrm{e}^{\alpha \mathcal{M}_{p}}=\mathrm{e}^{i p \varpi \alpha} P+\left(\mathbf{1}_{\mathfrak{H}_{\mathrm{at}}^{(p, 0)}}-P\right) \mathrm{e}^{\alpha \mathcal{M}_{p}^{\prime}}\left(\mathbf{1}_{\mathfrak{H}_{\mathrm{at}}^{(p, 0)}}-P\right)
$$

with

$$
\mathcal{M}_{p}^{\prime}:=\left(\mathbf{1}_{\mathfrak{H}_{\mathrm{at}}^{(p, 0)}}-P\right) \mathcal{M}_{p}\left(\mathbf{1}_{\mathfrak{H}_{\mathrm{at}}^{(p, 0)}}-P\right) .
$$

Using spectral properties of the operator

$$
\left(i \varpi p+\lambda^{2} \mathfrak{L}_{\mathcal{R}}^{*}+\frac{\eta}{2} \mathfrak{L}_{\mathrm{p}}^{*}\right) \in \mathcal{B}\left(\mathfrak{H}_{\mathrm{at}}^{(p, 0)}\right)
$$

and Kato's perturbation theory we deduce that

$$
\sigma\left(\left.\mathcal{M}_{p}^{\prime}\right|_{\operatorname{ran}\left(\mathbf{1}_{\mathfrak{H}_{\text {at }}^{(p, 0)}}-P\right)}\right) \subset\left(-\infty, C \lambda^{2}\right)+i \mathbb{R}
$$

for some constant $C \in \mathbb{R}^{+}$and sufficiently small $|\lambda|$. [It is similar to (4.93) or [1, Lemma 6.3] and it results from Assumption 4] Since the generator $\left.\mathcal{M}_{p}^{\prime}\right|_{\operatorname{ran}\left(\mathbf{1}_{\mathfrak{H}_{\mathrm{at}}^{(p, 0)}}-P\right)}$ acts on a finite dimensional space, this spectral condition implies that the corresponding semigroup is bounded. By (4.109), the semigroup $\left\{\mathrm{e}^{\alpha \mathcal{M}_{p}}\right\}_{\alpha \geq 0}$ is thus bounded.

The proof of $\left\|\mathrm{e}^{\alpha \tilde{\mathcal{K}}_{p}}\right\|_{\mathcal{B}(\hat{\mathcal{H}})} \leq C$ is performed in the same way. It is even simpler because the real part of the spectrum of $\tilde{\mathcal{K}}_{p}$ is strictly negative, by Theorem 4.15 (ii).

We now study the semigroup $\left\{\mathrm{e}^{\alpha \hat{\mathcal{G}}(i r)}\right\}_{\alpha \geq 0}$ via (4.95) and (4.98):

\section{Theorem 4.19 (Time-Uniform Approximations of $\left\{\mathrm{e}^{\alpha \hat{\mathcal{G}}(i r)}\right\}_{\alpha \geq 0}$ )}

There is a constant $C \in \mathbb{R}^{+}$such that, for all $\alpha \in \mathbb{R}_{0}^{+}, \hat{\psi}, \hat{\psi}^{\prime} \in \hat{\mathfrak{H}}_{\text {at }}$ and sufficiently small $|\lambda|$,

$$
\left|\left\langle\hat{\psi},\left(\mathrm{e}^{\alpha \hat{\mathcal{G}}(i r)}-\mathrm{e}^{\alpha \mathcal{K}_{0}} \mathcal{P}_{0,0}^{(\lambda, \eta)}-\mathrm{e}^{\alpha \tilde{\mathcal{K}}_{0}} \tilde{\mathcal{P}}_{0}^{(\lambda, \eta)}-\mathrm{e}^{\alpha \tilde{\mathcal{K}}_{-1}} \tilde{\mathcal{P}}_{-1}^{(\lambda, \eta)}\right) \hat{\psi}^{\prime}\right\rangle_{\hat{\mathcal{H}}}\right| \leq C\left(\varpi^{-2}+\lambda^{2} \mathrm{e}^{v \alpha}\right)\|\hat{\psi}\|_{\hat{\mathcal{H}}}\left\|\hat{\psi}^{\prime}\right\|_{\hat{\mathcal{H}}} .
$$

Moreover, if $\hat{\psi}^{\prime} \in \mathfrak{F}\left(\mathfrak{D} \otimes \Omega_{\mathcal{R}}\right)$ then

$$
\left|\left\langle\hat{\psi},\left(\mathrm{e}^{\alpha \hat{\mathcal{G}}(i r)}-\mathrm{e}^{\alpha \mathcal{K}_{0}}\right) \hat{\psi}^{\prime}\right\rangle_{\hat{\mathcal{H}}}\right| \leq C\left(\left(1+\varpi^{-2}\right)|\lambda|+\lambda^{2} \mathrm{e}^{v \alpha}\right)\|\hat{\psi}\|_{\hat{\mathcal{H}}}\left\|\hat{\psi}^{\prime}\right\|_{\hat{\mathcal{H}}} .
$$

Proof. The assertion is a direct consequence of (4.95), (4.98) and (4.104) combined with Lemmata 4.164.18,

\subsection{Proof of Theorem 3.1}

1. As the estimates leading to Theorem 3.1 are uniform w.r.t. the initial atomic state and the dynamical problems involved are well-posed, we consider, w.l.o.g, only the case of a faithful initial atomic state. This implies, in particular, that the density matrix $\rho_{\text {at }}$, and hence the operator $\mathfrak{Z}_{\text {at }}$, has an inverse. Let

$$
\Lambda^{(\lambda, \eta)}:=\left(\mathcal{P}_{0,0}^{(0,0)}\left(\eta \mathfrak{S} \mathfrak{Z}_{\mathrm{at}} \mathfrak{L}_{\mathrm{p}}^{*} \mathfrak{Z}_{\mathrm{at}}^{-1}+\lambda^{2} \mathfrak{Z}_{\mathrm{at}} \mathfrak{L}_{\mathcal{R}}^{*} \mathfrak{Z}_{\mathrm{at}}^{-1}\right) \mathcal{P}_{0,0}^{(0,0)}\right)^{*} \in \mathcal{B}(\hat{\mathcal{H}}) .
$$


By (4.76), observe that $\operatorname{ran}\left(\mathcal{P}_{0,0}^{(0,0)}\right)$ is equivalent to the space $\mathfrak{H}_{0}^{(0,0)}$ of 1 , Sections 4.4, 6.2]. In particular, $\Lambda^{(\lambda, \eta)}$ can in this way be identified with the operator $\Lambda^{(\lambda, \eta)} \equiv \Lambda_{0}^{(\lambda, \eta)}$ of [1, Section 6.2]. See also [1, Eq. (6.6)]. By Theorem 4.14 and (4.108),

$$
\left\|\left(\Lambda^{(\lambda, \eta)}\right)^{*}-\mathcal{K}_{0}\right\|_{\mathcal{B}(\hat{\mathcal{H}})} \leq C|\lambda|^{3}
$$

for some finite constant $C \in \mathbb{R}^{+}$not depending on $\lambda, \eta(|\lambda|$ sufficiently small). Using exactly the same arguments as in the proofs of [1, Lemma 6.4, Theorem 4.4], one verifies that, for any $\varepsilon \in(0,1)$,

$$
\left\|\mathrm{e}^{\alpha\left(\Lambda^{(\lambda, \eta)}\right)^{*}}-\mathrm{e}^{\alpha \mathcal{K}_{0}}\right\|_{\mathcal{B}(\hat{\mathcal{H}})} \leq C \min \left\{\alpha|\lambda|^{3},|\lambda|+\mathrm{e}^{-D \alpha \lambda^{2}}\right\} \leq C|\lambda|^{1-\varepsilon}
$$

for some constants $C, D \in \mathbb{R}^{+}$not depending on $\lambda, \eta(|\lambda|$ sufficiently small).

2. Now, we combine (4.111) with Theorems 4.8, 4.11, 4.19 to deduce that, for any initial faithful state $\omega_{\text {at }}$ with density matrix $\rho_{\text {at }} \in \mathfrak{D} \subset \mathcal{B}\left(\mathbb{C}^{d}\right)$, any observable $A \in \mathfrak{D} \subset \mathcal{B}\left(\mathbb{C}^{d}\right), \varepsilon \in(0,1)$ and sufficiently small $|\lambda|$,

$$
\left|\left\langle\Omega, U_{0, \alpha}\left(A \otimes \mathbf{1}_{\mathfrak{H}_{\mathcal{R}}} \Omega\right)\right\rangle_{\mathfrak{H}}-\left\langle\Omega, \mathrm{e}^{\alpha\left(\Lambda^{(\lambda, \eta)}\right)^{*}}\left(A \otimes \mathbf{1}_{\mathfrak{H}_{\mathcal{R}}} \Omega\right)\right\rangle_{\mathcal{H}}\right| \leq C_{\varpi, \varepsilon}\|A\|_{\mathcal{B}\left(\mathbb{C}^{d}\right)}|\lambda|^{1-\varepsilon},
$$

where $C_{\varpi, \varepsilon} \in \mathbb{R}_{0}^{+}$is a finite constant depending on $\varpi, \varepsilon$ but not on $\omega_{\text {at }}, A, \lambda, \eta$, and $\alpha$. Recall that $\left\{U_{s, t} \equiv\right.$ $\left.U_{s, t}^{(\lambda, \eta)}\right\}_{s, t \in \mathbb{R}}$ is the non-autonomous evolution family of Proposition 4.4, By (3.3),

$$
\left\langle\Omega, U_{0, \alpha}\left(A \otimes \mathbf{1}_{\mathfrak{H}_{\mathcal{R}}} \Omega\right)\right\rangle_{\mathfrak{H}}=\omega_{\text {at }}(\alpha)(A)
$$

Moreover, for any observable $A \in \mathfrak{D} \subset \mathcal{B}\left(\mathbb{C}^{d}\right)$,

$$
\omega_{\text {at }}(\alpha)(A)=\left\langle\rho_{\text {at }}(\alpha), A\right\rangle_{\mathfrak{H}_{\text {at }}}=\left\langle P_{\mathfrak{D}}\left(\rho_{\text {at }}(\alpha)\right), A\right\rangle_{\mathfrak{H}_{\text {at }}},
$$

see (2.5). Recall also that $\rho_{\text {at }}(\alpha)$ is, by definition, the density matrix of $\omega_{\text {at }}(\alpha)$ and $P_{\mathfrak{D}}$ is the orthogonal projection on the subspace $\mathfrak{D}(3.4)$ of block-diagonal matrices. Meanwhile, we infer from (4.108) that

$$
\Lambda^{(\lambda, \eta)}=\left(\mathcal{P}_{0,0}^{(0,0)} \mathrm{U}_{0,0}^{*} \mathfrak{Z}_{\text {at }}\left(\frac{\eta}{2} \mathfrak{L}_{\mathrm{p}}^{*}+\lambda^{2} \mathfrak{L}_{\mathcal{R}}^{*}\right) \mathfrak{Z}_{\mathrm{at}}^{-1} \mathrm{U}_{0,0} \mathcal{P}_{0,0}^{(0,0)}\right)^{*} .
$$

[1. Theorem 4.6 (i)] implies that $\mathfrak{H}_{\mathrm{at}}^{(0,0)}$ is an invariant space of

$$
\mathfrak{Z}_{\text {at }}\left(\frac{\eta}{2} \mathfrak{L}_{\mathrm{p}}^{*}+\lambda^{2} \mathfrak{L}_{\mathcal{R}}^{*}\right) \mathfrak{Z}_{\text {at }}^{-1} \in \mathcal{B}\left(\mathfrak{H}_{\text {at }}\right)
$$

and we deduce from (4.115) that

$$
\mathrm{e}^{\alpha\left(\Lambda^{(\lambda, \eta)}\right)^{*}} \mathcal{P}_{0,0}^{(0,0)}=\mathrm{U}_{0,0}^{*} \mathfrak{Z}_{\mathrm{at}} \mathrm{e}^{\alpha\left(\frac{\eta}{2} \mathfrak{L}_{\mathrm{p}}^{*}+\lambda^{2} \mathfrak{L}_{\mathcal{R}}^{*}\right)} \mathfrak{Z}_{\mathrm{at}}^{-1} \mathrm{U}_{0,0} \mathcal{P}_{0,0}^{(0,0)} .
$$

By (4.78), it follows that, for any observable $A \in \mathfrak{D} \subset \mathcal{B}\left(\mathbb{C}^{d}\right)$ and initial density matrix $\rho_{\text {at }} \in \mathfrak{D}$,

$$
\begin{aligned}
\left\langle\Omega, \mathrm{e}^{\alpha\left(\Lambda^{(\lambda, \eta)}\right)^{*}}\left(A \otimes \mathbf{1}_{\mathfrak{H}_{\mathcal{R}}} \Omega\right)\right\rangle_{\mathcal{H}} & =\left\langle\rho_{\mathrm{at}}^{1 / 2}, \mathfrak{Z}_{\mathrm{at}} \mathrm{e}^{\alpha\left(\frac{\eta}{2} \mathfrak{L}_{\mathrm{p}}^{*}+\lambda^{2} \mathfrak{L}_{\mathcal{R}}^{*}\right)} \mathfrak{Z}_{\mathrm{at}}^{-1} A \rho_{\mathrm{at}}^{1 / 2}\right\rangle_{\mathfrak{H}_{\mathrm{at}}} \\
& =\left\langle\rho_{\mathrm{at}}, \mathrm{e}^{\alpha\left(\frac{\eta}{2} \mathfrak{L}_{\mathrm{p}}^{*}+\lambda^{2} \mathfrak{L}_{\mathcal{R}}^{*}\right)} A\right\rangle_{\mathfrak{H}_{\mathrm{at}}} \\
& =\left\langle\mathrm{e}^{\alpha\left(\frac{\eta}{2} \mathfrak{L}_{\mathrm{p}}+\lambda^{2} \mathfrak{L}_{\mathcal{R}}\right)} \rho_{\mathrm{at}}, A\right\rangle_{\mathfrak{H}_{\mathrm{at}}} \\
& =\left\langle P_{\mathfrak{D}}\left(\mathrm{e}^{\alpha\left(\frac{\eta}{2} \mathfrak{L}_{\mathrm{p}}+\lambda^{2} \mathfrak{L}_{\mathcal{R}}\right)} \rho_{\mathrm{at}}\right), A\right\rangle_{\mathfrak{H}_{\mathrm{at}}} \\
& =\left\langle P_{\mathfrak{D}}\left(\mathrm{e}^{\alpha \tilde{\Lambda}^{(\lambda, \eta)}} \rho_{\mathrm{at}}\right), A\right\rangle_{\mathfrak{H}_{\mathrm{at}}},
\end{aligned}
$$

where

$$
\tilde{\Lambda}^{(\lambda, \eta)}=\frac{\eta}{2} \mathfrak{L}_{\mathrm{p}}+\lambda^{2} \mathfrak{L}_{\mathcal{R}}
$$

is the operator of [1, Theorem 4.6 (i)]. We used in the second equality the identities

$$
\mathcal{P}_{0,0}^{(0,0)}\left(\left(A \rho_{\mathrm{at}}^{1 / 2}\right) \otimes \Omega_{\mathcal{R}}\right)=\left(A \rho_{\mathrm{at}}^{1 / 2}\right) \otimes \Omega_{\mathcal{R}} \quad \text { and } \quad \mathrm{U}_{0,0}\left(\left(A \rho_{\mathrm{at}}^{1 / 2}\right) \otimes \Omega_{\mathcal{R}}\right)=A \rho_{\mathrm{at}}^{1 / 2}
$$


when $A \in \mathfrak{D}$. [Cf. definition (4.78).]

3. Therefore, Theorem 3.1 follows from Equations (4.112)-(4.116) and [1, Corollary 4.5, Eqs. (4.37)-(4.38)].

\section{Appendix}

1. For the reader's convenience, we review here one definition and a result from the theory of analytic families of closed operators used in Section 4.5 to study the analytic deformation of the Howland operator $\mathcal{G}$.

\section{Definition 5.1 (Families of type A)}

For an open subset $\mathcal{S}$ of $\mathbb{C}$, a family $\left\{F_{z}\right\}_{z \in \mathcal{S}}$ of closed operators defined on a Banach space $\mathcal{X}$ and with nonempty resolvent set is of type $A$ when $\operatorname{Dom}\left(F_{z}\right)=\mathcal{Y} \subset \mathcal{X}$ of $F_{z}$ is independent of $z \in \mathcal{S}$ and the map $z \mapsto F_{z} x$ is strongly analytic on $\mathcal{S}$ for all $x \in \mathcal{Y}$.

Families of type A are useful in the context of Kato's perturbation theory as they form a special case of analytic family (in the sense of Kato). In our proofs, we use the following well-known result on type A families (see [12, Sect. VII.1, Theorem 1.3]):

\section{Lemma 5.2 (Type A families and analycity of resolvents)}

Let $\left\{F_{z}\right\}_{z \in \mathcal{S}}$ be a closed operator family of type $A$ and let $\zeta \in \varrho\left(F_{z_{0}}\right)$, where $\varrho\left(F_{z_{0}}\right)$ is the resolvent set of $F_{z_{0}}$ with $z_{0} \in \mathcal{S}$. Then the map $z \mapsto\left(\zeta-F_{z}\right)^{-1}$ is analytic in some neighborhood of $z_{0}$.

2. We used a representation of strongly continuous one-parameter semigroups w.r.t. resolvents. Indeed, resolvents and semigroups are related to each other through the Laplace transform (see, e.g., [25, Chap. III, Corollary 5.15]):

\section{Lemma 5.3 (Semigroups as Laplace transforms)}

Let $A$ be a (possibly unbounded) generator of a strongly continuous semigroup $\left\{\mathrm{e}^{\alpha A}\right\}_{\alpha \geq 0}$ on a Banach space $\mathcal{X}$ with growth bound $\varsigma \in \mathbb{R} \cup\{-\infty\}$. Then, for all $x \in \operatorname{Dom}(A), w>\varsigma$ and $\alpha \in \mathbb{R}^{+}$,

$$
\mathrm{e}^{\alpha A} x=\lim _{N \rightarrow \infty}\left\{\frac{1}{2 \pi i} \int_{w-i N}^{w+i N} \mathrm{e}^{\alpha \zeta}(\zeta-A)^{-1} x \mathrm{~d} \zeta\right\} .
$$

3. In Kato's perturbation theory, a perturbed Riesz projection $P(y)$ is related to the (non-perturbed Riesz) projection $P(0)$ through some bounded operator $U(y)$ with bounded inverse $V(y)$ by the relation $P(y)=$ $U(y) P(0) V(y)$, provided the coupling constant $y \in \mathbb{R}$ measuring the strength of the perturbation is small enough in absolute value. This fact is used to prove Lemma 4.18 The following relations between pairs of projections (see [12, I.4.6. p. 33]) are important in that proof: Let $P, Q$ be two projections acting on a Banach space $\mathcal{X}$. Then, the bounded operator

$$
U^{\prime}:=Q P+(1-Q)(1-P)
$$

maps $\operatorname{ran}(P)$ into $\operatorname{ran}(Q)$, whereas

$$
V^{\prime}:=P Q+(1-P)(1-Q)
$$

maps $\operatorname{ran}(Q)$ into $\operatorname{ran}(P)$. Moreover, $V^{\prime} U^{\prime}=U^{\prime} V^{\prime}=1-\mathcal{R}$, where $\mathcal{R}:=(P-Q)^{2}$. In Kato's perturbation theory, the operator $(P(y)-P(0))$ has typically a small norm for small $|y|$ and $(1-\mathcal{R})^{-1 / 2}$ exists as an absolutely convergent power series in $\mathcal{R}$. Under this assumption and since $\mathcal{R}$ always commutes with $P$ and $Q$, we can relate both projections to each other, as explained above:

\section{Lemma 5.4 (Pairs of near projections)}

Let $P, Q$ be two projections and $\mathcal{R}:=(P-Q)^{2}$. If $(1-\mathcal{R})^{-1 / 2}$ exists and is bounded then the bounded operator

$$
U:=U^{\prime}(1-\mathcal{R})^{-1 / 2}=(1-\mathcal{R})^{-1 / 2} U^{\prime}
$$


maps $\operatorname{ran}(P)$ into $\operatorname{ran}(Q)$, whereas

$$
V:=V^{\prime}(1-\mathcal{R})^{-1 / 2}=(1-\mathcal{R})^{-1 / 2} V^{\prime}
$$

maps $\operatorname{ran}(Q)$ into $\operatorname{ran}(P)$. Moreover, $V U=U V=1$, i.e., $V=U^{-1}$, and $P=V Q U, Q=U P V$.

Acknowledgments: We would like to thank Volker Bach for his support as well as Matthias Westrich for discussions and his help in Section 4.2. We thank the referees and the editor for useful hints. This research is supported by a grant of the "Inneruniversitäre Forschungsförderung" of the Johannes Gutenberg University, by the agencies MINECO and FAPESP under Grants MTM2010-16843 and 2013/13215-5 as well as by the Basque Government through the grant IT641-13 and the BERC 2014-2017 program and by the Spanish Ministry of Economy and Competitiveness MINECO: BCAM Severo Ochoa accreditation SEV-2013-0323.

\section{REFERENCES}

[1] J.-B. Bru, W. de Siqueira Pedra, M. Westrich. Characterization of the Quasi-Stationary State of an Impurity Driven by Monochromatic Light I - The Effective Theory, Annales Henri Poincaré, 13(6):1305-1370, 2012.

[2] E. Snitzer and C.G. Young. Lasers. Volume 2 of A Series of Advances. Edward Arnold, London, 1968.

[3] A. Aspect, G. Grynberg, C. Fabre. Introduction to Quantum Optics, Volume XXIX. Cambridge Univ. Press, 2010.

[4] R.H. Dicke. Coherence in Spontaneous Radiation Processes. Phys. Rev., 93:99-110, 1954.

[5] K. Hepp and E.H. Lieb. Phase Transitions in Reservoir Driven Open Systems with Applications to Lasers and Superconductors. Helv. Phys. Acta, 46:573-603, 1973.

[6] K. Hepp and E.H. Lieb. On the Superradiant Phase Transition for Molecules in a Quantized Electromagnetic Field: the Dicke Maser Model. Ann. of Phys., 76:360-404, 1973.

[7] K. Hepp and E.H. Lieb. The Laser: A Reversible Quantum Dynamical System with Irreversible Classical Macroscopic Motion. In: J. Moser (editor), Dynamical Systems, Theory and Applications, Lecture Notes in Physics, 38:178-208, 1975.

[8] G. Alli and G.L. Sewell. New Methods and Structures in the Theory of the Multi-Mode Dicke Laser Model. J. Math. Phys., 36:5598-5626, 1995.

[9] E. Snitzer and C.G. Young. Lasers, Volume 2 of A Series of Advances. Edward Arnold, London, 1968.

[10] G.L. Sewell. Quantum Mechanics and its Emergent Macrophysics. Princeton University Press, 2002.

[11] J. McKeever, A. Boca, A. D. Boozer, J. R. Buck and H. J. Kimble. Experimental realization of a one-atom laser in the regime of strong coupling. Nature, 425:268-271, 2003.

[12] T. Kato. Perturbation Theory for Linear Operators, 2nd Ed. Springer-Verlag, Berlin-Heidelberg-New-York, 1980.

[13] V. Bach, W. de Siqueira Pedra, M. Merkli, I. M. Sigal. Suppression of Decoherence by Periodic Forcing. Journal of Statistical Physics, 155(6):1271-1298, 2014.

[14] J. Derezinski and R. Früboes. Fermi Golden Rule and Open Quantum Systems. In Open Quantum Systems III, Volume 1882, pages 67-116. Springer-Verlag, 2006.

[15] V. Jakšić and C.-A. Pillet. Non-Equilibrium Steady States of Finite Quantum Systems Coupled to Thermal Reservoirs. Commun. Math. Phys., 226:131-162, 2002.

[16] H. Spohn. An Algebraic Condition for the Approach to Equilibrium of an Open $n$-Level System. Lett. in Math. Phys., 2:33-38, 1977. 
[17] W. K. Abou Salem. On the Quasi-Static Evolution of Nonequilibrium Steady States. Ann. Henri Poincaré, $8(3): 569-596,2007$.

[18] W. K. Abou Salem, J. Fröhlich. Cyclic Thermodynamic processes and Entropy Production. Journal of Statistical Physics, 126(3):431-466, 2007.

[19] M. Westrich. Long-Time Dynamics of Open Quantum Systems. PhD's thesis, Aahrus University, Department of Mathematics, 2011.

[20] O. Bratteli and D.W. Robinson, Operator Algebras and Quantum Statistical Mechanics, Vol. II, 2 nd ed. Springer-Verlag, New York, 1996.

[21] O. Bratteli and D.W. Robinson, Operator Algebras and Quantum Statistical Mechanics, Vol. I, 2 nd ed. Springer-Verlag, New York, 1996.

[22] J.-B. Bru, W. de Siqueira Pedra and C. Hertling, Heat Production of Non-Interacting Fermions Subjected to Electric Fields, Comm. Pure Appl. Math., 68(6):964-1013, 2015.

[23] A. Pazy, Semigroups of Linear Operators and Applications to Partial Differential Equations. Applied Mathematical Sciences 44, Springer, New-York, 1983.

[24] V. Jakšić and C.-A. Pillet. On a model for quantum friction II. Fermi's golden rule and dynamics at positive temperature. Commun. Math. Phys., 176:619-644, 1996.

[25] K.-J. Engel and R. Nagel. One-Parameter Semigroups for Linear Evolution Equations. Springer-Verlag, Berlin-Heidelberg-New-York, 2000. 